\title{
The 21-SPONGE H I Absorption Line Survey. I. The Temperature of Galactic H I
}

\author{
Claire E. Murray ${ }^{1,2}$ (1) , Snežana Stanimirović ${ }^{1}$, W. M. Goss ${ }^{3}$ (1), Carl Heiles ${ }^{4}$, John M. Dickey $^{5}$ (1), Brian Babler ${ }^{1}$ (1), and \\ Chang-Goo $\mathrm{Kim}^{6,7}$ (i) \\ ${ }^{1}$ Department of Astronomy, University of Wisconsin, Madison, WI 53706, USA; clairemurray56@ gmail.com \\ ${ }^{2}$ Space Telescope Science Institute, 3700 San Martin Drive, Baltimore, MD 21218, USA \\ ${ }^{3}$ National Radio Astronomy Observatory, P.O. Box O, 1003 Lopezville, Socorro, NM 87801, USA \\ ${ }^{4}$ Radio Astronomy Lab, University of California, Berkeley, 601 Campbell Hall, Berkeley CA 94720, USA \\ ${ }^{5}$ University of Tasmania, School of Maths and Physics, Hobart, TAS 7001, Australia \\ ${ }^{6}$ Department of Astrophysical Sciences, Princeton University, Princeton, NJ 08544, USA \\ ${ }^{7}$ Center for Computational Astrophysics, Flatiron Institute, New York, NY 10010, USA \\ Received 2018 June 2; revised 2018 August 2; accepted 2018 August 3; published 2018 September 27
}

\begin{abstract}
We present $21 \mathrm{~cm}$ Spectral Line Observations of Neutral Gas with the VLA (21-SPONGE), a Karl G. Jansky Very Large Array (VLA) large project ( $600 \mathrm{hr}$ ) for measuring the physical properties of Galactic neutral hydrogen (H I). 21-SPONGE is distinguished among previous Galactic H I studies as a result of (1) its exceptional optical depth sensitivity $\left(\sigma_{\tau}<10^{-3}\right.$ per $0.42 \mathrm{~km} \mathrm{~s}^{-1}$ channel over 57 lines of sight), (2) matching $21 \mathrm{~cm}$ emission spectra with the highest possible angular resolution $\left(\sim 4^{\prime}\right)$ from the Arecibo Observatory, and (3) detailed comparisons with numerical simulations for assessing observational biases. We autonomously decompose $21 \mathrm{~cm}$ spectra and derive the physical properties (i.e., spin temperature, $T_{s}$, and column density) of the cold neutral medium (CNM; $T_{s}<250 \mathrm{~K}$ ), thermally unstable medium (UNM; $250 \mathrm{~K}<T_{s}<1000 \mathrm{~K}$ ), and warm neutral medium (WNM; $T_{s}>1000 \mathrm{~K}$ ) simultaneously. Of the total H I mass observed, $50 \%$ is detected in both absorption and emission. The CNM makes up the majority of the absorbing gas $(56 \% \pm 10 \%)$ and $28 \%$ of the total $\mathrm{H} \mathrm{I}$ mass including gas detected only in emission. We find that $20 \%$ of the total $\mathrm{H} \mathrm{I}$ mass is thermally unstable $(41 \% \pm 10 \%$ of $\mathrm{H} \mathrm{I}$ detected in absorption), with no significant variation with Galactic latitude. Finally, although the WNM makes up 52\% of the total H I mass, we detect little evidence for WNM absorption with $1000 \mathrm{~K}<T_{s}<4000 \mathrm{~K}$. Following spectral modeling, we detect a stacked residual absorption feature corresponding to WNM with $T_{s} \sim 10^{4} \mathrm{~K}$. We conclude that excitation in excess of collisions likely produces significantly higher WNM $T_{s}$ than predicted by steady-state models.
\end{abstract}

Key words: ISM: clouds - ISM: structure - radio lines: ISM

Supporting material: figure sets

\section{Introduction}

The formation of stars and the evolution of galaxies rely on the cycle of interstellar matter (ISM) between supernovaexpelled plasma and molecule-rich gas. At the center of this cycle is neutral hydrogen (HI), the fundamental fuel for starforming clouds whose physical conditions comprise key constraints for theoretical models.

Following the first astronomical observations of absorption and emission via the $21 \mathrm{~cm}$ transition of $\mathrm{HI}$ (Ewen \& Purcell 1951; Muller \& Oort 1951; Hagen et al. 1955), clear differences in the observed velocity structure between $21 \mathrm{~cm}$ emission and absorption were attributed to significant variations in the temperature and density of the gas along the line of sight (LOS; e.g., Clark 1965; Dickey et al. 1978). Theoretical models of steady-state ISM heating and cooling quantified the nature of this thermal phase structure, predicting two thermally stable phases: the cold neutral medium (CNM) and warm neutral medium (WNM), with density and kinetic temperatures of $\left(n, T_{k}\right)=\left(7-70 \mathrm{~cm}^{-3}\right.$, $60-260 \mathrm{~K})$, and $\left(n, T_{k}\right)=\left(0.2-0.9 \mathrm{~cm}^{-3}, 5000-8300 \mathrm{~K}\right)$, respectively (McKee \& Ostriker 1977; Wolfire et al. 2003). However, subsequent analytical models and numerical simulations determined that time-dependent or dynamical processes such as turbulence and supernova shocks are likely very important and will generate a significant amount of thermally unstable gas (UNM) in the intervening parameter space between CNM and WNM, thus throwing into question the validity of the steady-state paradigm of the ISM (e.g., Dalgarno \& McCray 1972; Vázquez-Semadeni et al. 2000; Audit \& Hennebelle 2005).

However, the physical properties, mass fractions, and ionization state of the diffuse neutral gas phases (i.e., WNM and UNM) are particularly sensitive to macro- and microphysical heating and cooling processes (Heiles \& Troland 2003b). These include magnetic wave dissipation (e.g., Ferrière et al. 1988), magnetic reconnection (e.g., Vishniac \& Lazarian 1999), turbulence (e.g., Audit \& Hennebelle 2005), supernovae (e.g., Mac Low et al. 2005), diffusion of photons from H II regions, and diffusion of low-energy cosmic rays and X-rays from time-dependent stellar phenomena. As these processes originate from sources on a huge range of physical and temporal scales, understanding them as part of a self-consistent model of the ISM has proven challenging.

Furthermore, observational constraints for the properties of the UNM and WNM have been historically limited by insufficient observational capabilities. To constrain the optical depth and excitation (or spin) temperature of $\mathrm{H} \mathrm{I}$-crucial for determining the thermodynamic state of the gas-measurements of both emission and absorption at $21 \mathrm{~cm}$ are necessary. Due to their low densities, detecting the absorbing properties of the WNM and UNM requires extremely high sensitivity to H I optical depth. For example, past observations of $21 \mathrm{~cm}$ 
absorption with single-dish and interferometric telescopes were primarily sensitive to detecting absorption by the CNM with $T_{s}=60-80 \mathrm{~K}$ (Hughes et al. 1971; Radhakrishnan et al. 1972; Crovisier et al. 1978). Improved sensitivity to optical depth revealed absorption by $\mathrm{HI}$ with warmer temperatures, up to 600 K (Lazareff 1975; Dickey et al. 1977). Only a handful of detections of WNM with $T_{s} \gtrsim 1000 \mathrm{~K}$ exist (Carilli et al. 1998; Dwarakanath et al. 2002; Murray et al. 2015).

Considering the expense of high-sensitivity absorption measurements, and the fact that they are limited by the availability of sources of background continuum radiation, warm HI (i.e., UNM and WNM) properties are typically indirectly estimated from $21 \mathrm{~cm}$ emission alone. Kinetic temperatures inferred from the Gaussian line widths of decomposed $21 \mathrm{~cm}$ emission profiles indicate that a significant fraction of the H I mass is thermally unstable, with $T_{s} \sim 3000 \mathrm{~K}$ (e.g., Verschuur \& Magnani 1994; Haud \& Kalberla 2007). For example, following the Gaussian decomposition of all-sky H I emission survey data from the Effelsberg-Bonn H I Survey (EBHIS; Winkel et al. 2016) and the Galactic All-Sky Survey (GASS; McClure-Griffiths et al. 2009; Kalberla et al. 2010; Kalberla \& Haud 2015), Kalberla \& Haud (2018) concluded that $41 \%$ of $\mathrm{H} \mathrm{I}$ in the local ISM $\left(\left|v_{\mathrm{LSR}}\right|<8 \mathrm{~km} \mathrm{~s}^{-1}\right)$ is in the "lukewarm" (a.k.a. thermally unstable) medium. The Millennium Arecibo $21 \mathrm{~cm}$ AbsorptionLine Survey detected absorption from gas with excitation temperatures of $\sim 10-600 \mathrm{~K}$ and inferred that $\sim 48 \%$ of the remaining material detected only in emission (i.e., $\sim 30 \%$ of the total column density) is thermally unstable (Heiles \& Troiland $2003 \mathrm{~b}$ ). From a high-sensitivity survey of $21 \mathrm{~cm}$ absorption toward 35 sources, Roy et al. 2013b estimated that at least $28 \%$ of $\mathrm{HI}$ is unstable. However, this result is based on $\mathrm{H} \mathrm{I}$ emission data from the Leiden Argentine Bonn (LAB; Kalberla et al. 2005) survey, whose $36^{\prime}$ resolution probes much larger scales and thus different $\mathrm{HI}$ populations than their subarcminute interferometric absorption measurements.

Improving on previous observational efforts to constrain the physical properties of the diffuse $\mathrm{HI}$, including the UNM mass fraction, requires expanded samples of $21 \mathrm{~cm}$ absorption lines at high sensitivity and careful attention to systematic uncertainties in analysis techniques.

\subsection{The 21-SPONGE Survey}

In this paper, we present the final data products from the largest survey for Galactic H I absorption to date at the Karl G. Jansky Very Large Array (VLA), titled $21 \mathrm{~cm}$ Observations of Neutral Gas with the (E)VLA (21-SPONGE). With superb sensitivity to $21 \mathrm{~cm}$ absorption, in combination with $21 \mathrm{~cm}$ emission from the Arecibo Observatory, 21-SPONGE is sensitive to CNM, UNM, and WNM temperatures and densities in the Galactic ISM. In Murray et al. (2015, hereafter M15), we presented the observation and data analysis strategies for 21-SPONGE, as well as preliminary analysis of $21 \mathrm{~cm}$ spectral line pairs. We demonstrated that the exceptional optical depth sensitivity of 21-SPONGE $\left(\sigma_{\tau}<0.001\right.$ per $0.42 \mathrm{~km} \mathrm{~s}^{-1}$ channel) enables direct detections of $\mathrm{HI}$ spin temperatures higher than previous observational studies by more than a factor of 2 (e.g., HT03).

Following the first half of the 21-SPONGE survey, we detected little evidence for WNM with $T_{s}>1000 \mathrm{~K}(<10 \%$ by number; M15). To improve sensitivity to shallow, broad absorption features further, we adapted a spectral stacking method to Galactic HI spectral line pairs and detected a pervasive population of WNM gas with $T_{s}=7200_{-1200}^{+1800} \mathrm{~K}$ (Murray et al. 2014). This excitation temperature is significantly higher than predictions from standard ISM models based on collisional H I excitation (e.g., Liszt 2001) and suggests that additional excitation mechanisms such as resonant Ly $\alpha$ scattering (i.e., the Wouthuysen-Field (WF) effect; Wouthuysen 1952; Field 1958) are important for determining the thermodynamic properties of diffuse, neutral gas. Furthermore, enhanced $21 \mathrm{~cm}$ excitation has important implications for understanding $\mathrm{H}$ I signals from early epochs of cosmic time when HI dominated the baryonic content of the universe (Pritchard \& Loeb 2012).

With the full 21-SPONGE survey now complete, our goal is to measure the mass distribution of $\mathrm{HI}$ as a function of temperature in the Galactic ISM. To prepare for this effort, in Murray et al. (2017) we considered the biases imposed by our analysis techniques in detail. Specifically, we analyzed synthetic H I absorption and emission spectral lines from a high-resolution, three-dimensional hydrodynamical simulation (Kim et al. 2013, 2014, hereafter KOK13 and KOK14) to assess the power of our observational methods for revealing the inherent state of the ISM. For the first time, we computed the completeness of HI structure recovery by Gaussian spectral line features and quantified the decline in completeness with latitude due to velocity crowding. Furthermore, we showed that the physical gas properties inferred from $21 \mathrm{~cm}$ spectral lines agree with the "true" simulated values within a factor of $<2$ for the majority of gas structures. We also identified a population of synthetic spectral features in KOK14, which are inconsistent with the properties of $21 \mathrm{~cm}$ spectra from 21-SPONGE, motivating improved treatment of $\mathrm{H}$ I excitation and feedback from star formation (Murray et al. 2017).

In this work, we will compare gas properties inferred from the KOK14 synthetic spectra to underlying physical properties of the KOK13 simulation to estimate the bias imposed by our spectral analysis methods on the overall mass distribution of $\mathrm{HI}$ as a function of temperature. Whereas in Murray et al. (2017) we focused on a subset of the KOK13 simulationconsidering individual gas structures selected along simulated LOSs and how their inherent properties were recovered by individual spectral line features-in this work, we consider all gas along each simulated LOS. We will then use these comparisons to estimate uncertainties in the observed 21SPONGE distribution to produce new constraints on the mass distribution of $\mathrm{HI}$ as a function of temperature.

This paper is organized as follows. In Section 2, we discuss the 21-SPONGE observations and synthetic data products used in our analysis. In Section 3, we discuss our revised Gaussian decomposition and radiative transfer approach, derived from HT03 and Murray et al. (2017). In Section 4, we present the results of our analysis of 21-SPONGE, including comparison with synthetic spectra from KOK14. In Section 6, we discuss these results, and we summarize our conclusions in Section 7.

\section{Data}

\subsection{VLA $21 \mathrm{~cm}$ Absorption Spectra}

The observing strategy for the 21-SPONGE H I absorption is described in M15 and summarized here for clarity. Our targets are bright $\left(S_{1.4 \mathrm{GHz}}>3 \mathrm{Jy}\right)$, radio continuum sources from the NRAO/VLA Sky Survey (NVSS; Condon et al. 1998) at high 
Galactic latitude (most at $|b|>10^{\circ}$, with a sample at low latitude for comparison) with compact angular sizes $\left(<1^{\prime}\right)$ to minimize the complexity of the observed $\mathrm{HI}$ profiles and to avoid resolving substantial continuum flux.

For all VLA observations, we used three separate $500 \mathrm{kHz}$ bands with $1.95 \mathrm{kHz}$ channel spacing centered on the $\mathrm{H} \mathrm{I}$ line $(1.42040575 \mathrm{GHz})$ and $\pm 1.5 \mathrm{MHz}$, respectively. We used the offline bands to avoid strong $\mathrm{HI}$ lines at Galactic velocities in the directions of our calibrator sources and to perform bandpass calibration via frequency switching. Bandpass calibration is of particular importance for 21-SPONGE, as our primary interest is to detect broad, shallow absorption lines associated with high-temperature H I. For details on our bandpass calibration strategies, we refer the reader to Section 2.2 of M15.

We reduced all 21-SPONGE data using the Astronomical Image Processing System (AIPS ${ }^{8}$; Greisen 2003). For a full description of the data reduction strategy, please see Section 2 of M15. For each source, we produce a cleaned, calibrated data cube and continuum image. We then extract the absorption spectrum from the pixel of maximum flux density and divide by the continuum flux density at the pixel location to compute $\exp (-\tau(v))$. Our channel spacing of $1.95 \mathrm{kHz}$ at the $\mathrm{HI}$ frequency $\left(0.42 \mathrm{~km} \mathrm{~s}^{-1}\right.$ channel spacing in velocity) corresponds to a velocity resolution of $0.5 \mathrm{~km} \mathrm{~s}^{-1}$ (Rohlfs \& Wilson 2004).

Of the original 58 target sources from the 21-SPONGE observing program, ${ }^{9} 10$ were removed upon inspection of preliminary data products for being overly resolved (three sources), displaying saturated absorption (two sources), or for not receiving integration time following the conclusion of the observing program (five sources). An additional nine sources were resolved into multiple continuum peaks, thereby providing additional sources for extracting $\mathrm{H}$ I absorption, albeit at degraded sensitivity due to the loss of continuum flux density. A gallery of continuum images for the 48 final target fields, demonstrating the range of complexity in the source structure, is included in the Appendix (Figure Set 12). Overall, we extracted 57 H I spectra from the 48 targets, including the spectra extracted from multiple continuum peaks (denoted by $A, B, C$ lettering). Figure 1 displays an all-Northern-sky map of H I column density from EBHIS, with the 57 final 21-SPONGE LOS coordinates overlaid. Table 1 displays detailed source information, including coordinates, NVSS flux density (Condon et al. 1998), and rms noise in optical depth $\left(\sigma_{\tau}\right)$ computed from offline channels $\left(0.42 \mathrm{~km} \mathrm{~s}^{-1}\right.$ channel spacing). We include source information for the overly resolved and saturated sources at the bottom of Table 1 .

In comparison with Table 2 of M15, we have improved $\sigma_{\tau}$ for many sources by including additional integration time and/ or reprocessing the original files. Generally, $\sigma_{\tau}<1 \times 10^{-3}$ (median value $=9 \times 10^{-4}$ ), which makes 21-SPONGE among the highest sensitivity surveys for $\mathrm{H}$ I absorption ever undertaken and covers more sources than previous highsensitivity surveys by almost a factor of 2 (e.g., Roy et al. 2013a). The outliers are due to degraded sensitivity from lack of flux density in the cases of our nine resolved sources. Overall, exceptional sensitivity to optical depth makes 21SPONGE sensitive to absorption by $\mathrm{HI}$ in all stable and thermally unstable ISM phases according to predictions from steady-state ISM models.

\footnotetext{
http://www.aips.nrao.edu/

9 VLA project codes: 10C-196, 12A-256, 13A-205.
}

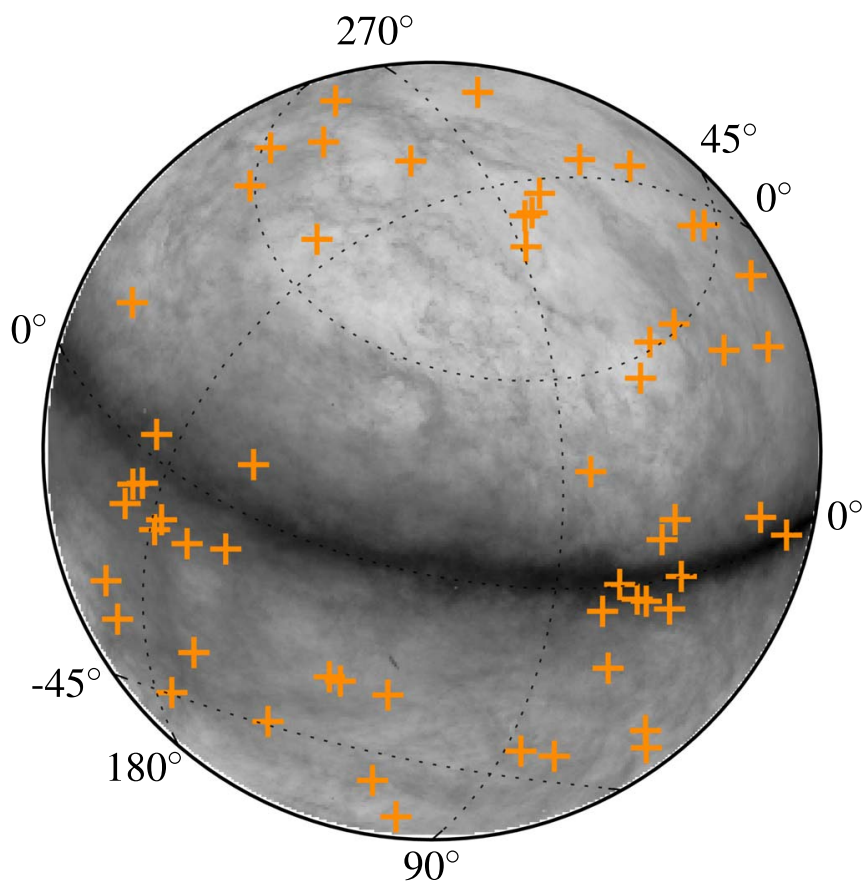

Figure 1. All-Northern-sky $N(\mathrm{HI})$ map from EBHIS in zenith-equal-area projection (Winkel et al. 2016), with coordinates for the 57 21-SPONGE LOS overlaid (orange pluses). Grid lines denote Galactic coordinates.

In M15, we demonstrated excellent agreement between 21-SPONGE and other H I absorption studies by comparing the integrated H I optical depths for sources that overlap with the Millennium Arecibo H I Absorption Line Survey (Heiles \& Troland 2003a, hereafter HT03), Stanimirović \& Heiles (2005), and Roy et al. (2013a). Of our 48 targets, we overlap with 22/ 78 from HT03, 9/35 from Roy et al. (2013a), and 9/104 from Mohan et al. (2004). In Table 1 of M15, we summarized these and other external surveys. We find consistent agreement with these studies at the level of our uncertainties.

All 21-SPONGE VLA spectra will be made publicly available and are accessible via their permanent Digital Object Identifier (DOI) at this link: 10.7910/DVN/BWFKL6.

\subsection{Matching H I Emission Spectra}

To estimate the temperatures and column densities of $\mathrm{H} \mathrm{I}$ structures using radiative transfer calculations, we need information about the brightness temperature of $\mathrm{HI}$ probed by our VLA absorption spectra. Observing $\mathrm{H} \mathrm{I}$ emission on the same angular scale as the $\mathrm{H}$ I absorption measurement is ideal; however, these measurements are prohibitively expensive to conduct at an interferometric facility such as the VLA. Therefore, we obtain the expected H I brightness temperature spectra $\left(T_{B, \text { exp }}(v)\right)$ along the same LOS as the VLA targets by interpolating emission spectra from neighboring LOSs across the target position, following the strategy outlined by HT03. We obtain $21 \mathrm{~cm}$ emission data from the $305 \mathrm{~m}$ Arecibo Observatory, whose $\sim 4^{\prime}$ beam at $1.4 \mathrm{GHz}$ allows us to minimize the effects of mismatched beam sizes on interpreting H I spectra. A total of 31 sources were observed as part of project A2770 at Arecibo, and 11 sources were obtained from publicly available data from HT03. Emission spectra for the remaining five sources, which lie outside of the Arecibo field of view, were obtained from the next highest resolution survey 
Table 1

VLA Observation Information

\begin{tabular}{|c|c|c|c|c|c|c|c|c|c|}
\hline $\begin{array}{l}\text { Source } \\
\text { (name) }\end{array}$ & $\begin{array}{l}\text { R.A. (J2000) } \\
\text { (hh:mm:ss) }\end{array}$ & $\begin{array}{l}\text { Decl. (J2000) } \\
\text { (dd:mm:ss) }\end{array}$ & $\begin{array}{c}l \\
\left({ }^{\circ}\right)\end{array}$ & $\begin{array}{c}b \\
\left({ }^{\circ}\right)\end{array}$ & $\underset{(\mathrm{Jy})}{S_{1.4 \mathrm{GHz}}}$ & $\begin{array}{l}\text { Synthesized Beam } \\
\qquad\left(" \times^{\prime \prime}\right)\end{array}$ & $\begin{array}{c}\sigma_{\tau} \\
\left(\times 10^{-3}\right)\end{array}$ & $\tau_{\text {peak }}$ & $\int_{\left(\mathrm{km} \mathrm{s}^{-1}\right)} \tau d v$ \\
\hline $\mathrm{J} 0022$ & $00: 22: 25.4$ & $+00: 14: 56.2$ & 107.462 & -61.748 & 3.01 & $2.2 \times 1.4$ & 0.8 & $0.025 \pm 0.001$ & $0.148 \pm 0.003$ \\
\hline $3 \mathrm{C} 018 \mathrm{~A}$ & $00: 40: 50.7$ & $+10: 03: 05.0$ & 118.623 & -52.732 & 4.60 & $15.5 \times 13.7$ & 1.5 & $0.623 \pm 0.004$ & $2.404 \pm 0.006$ \\
\hline $3 \mathrm{C} 018 \mathrm{~B}$ & $00: 40: 49.5$ & $+10: 03: 50.0$ & 118.616 & -52.719 & 4.60 & $15.5 \times 13.7$ & 2.4 & $0.642 \pm 0.006$ & $2.305 \pm 0.010$ \\
\hline $3 \mathrm{C} 041 \mathrm{~A}$ & $01: 26: 44.8$ & $+33: 13: 02.3$ & 131.379 & -29.075 & 3.71 & $1.7 \times 1.4$ & 2.4 & $0.039 \pm 0.004$ & $0.343 \pm 0.009$ \\
\hline $3 \mathrm{C} 041 \mathrm{~B}$ & $01: 26: 43.8$ & $+33: 13: 21.8$ & 131.374 & -29.070 & 3.71 & $1.7 \times 1.4$ & 3.8 & $0.057 \pm 0.006$ & $0.317 \pm 0.014$ \\
\hline $3 \mathrm{C} 48$ & $01: 37: 41.3$ & $+33: 09: 35.1$ & 133.963 & -28.719 & 16.02 & $1.3 \times 1.2$ & 0.7 & $0.050 \pm 0.001$ & $0.379 \pm 0.003$ \\
\hline 4C 15.05 & 02:04:50.4 & $+15: 14: 11.0$ & 147.930 & -44.043 & 4.07 & $3.3 \times 3.0$ & 0.6 & $0.086 \pm 0.001$ & $0.723 \pm 0.003$ \\
\hline $3 \mathrm{C} 78$ & 03:08:26.2 & $+04: 06: 39.0$ & 174.858 & -44.514 & 5.75 & $4.1 \times 2.2$ & 2.2 & $1.366 \pm 0.006$ & $4.992 \pm 0.009$ \\
\hline 4C 16.09 & 03:18:57.8 & $+16: 28: 32.7$ & 166.636 & -33.596 & 8.03 & $1.4 \times 1.2$ & 0.6 & $0.539 \pm 0.002$ & $3.019 \pm 0.003$ \\
\hline $3 \mathrm{C} 111 \mathrm{~A}$ & $04: 18: 21.3$ & $+38: 01: 35.8$ & 161.676 & -8.820 & 7.73 & $13.0 \times 5.3$ & 1.7 & $0.888 \pm 0.005$ & $10.167 \pm 0.010$ \\
\hline $3 \mathrm{C} 111 \mathrm{~B}$ & $04: 18: 30.4$ & $+38: 02: 30.4$ & 161.686 & -8.788 & 4.31 & $13.0 \times 5.3$ & 1.6 & $1.150 \pm 0.005$ & $10.225 \pm 0.009$ \\
\hline $3 \mathrm{C} 111 \mathrm{C}$ & $04: 18: 15.5$ & $+38: 00: 48.2$ & 161.671 & -8.843 & 2.92 & $13.0 \times 5.3$ & 3.0 & $1.125 \pm 0.009$ & $11.367 \pm 0.016$ \\
\hline $3 \mathrm{C} 120$ & 04:33:11.1 & $+05: 21: 15.6$ & 190.373 & -27.397 & 3.44 & $4.7 \times 4.3$ & 0.9 & $2.033 \pm 0.003$ & $10.625 \pm 0.004$ \\
\hline $3 \mathrm{C} 123 \mathrm{~A}$ & 04:37:04.9 & $+29: 40: 10.2$ & 170.584 & -11.660 & 49.73 & $20.3 \times 5.3$ & 0.6 & $1.750 \pm 0.002$ & $9.034 \pm 0.003$ \\
\hline $3 \mathrm{C} 123 \mathrm{~B}$ & 04:37:04.0 & $+29: 40: 28.2$ & 170.578 & -11.659 & 49.73 & $20.3 \times 5.3$ & 0.7 & $1.783 \pm 0.002$ & $8.795 \pm 0.004$ \\
\hline $3 \mathrm{C} 132$ & $04: 56: 43.5$ & $+22: 49: 16.3$ & 178.862 & -12.522 & 3.43 & $1.8 \times 1.5$ & 1.1 & $1.614 \pm 0.005$ & $7.371 \pm 0.007$ \\
\hline $3 C 133$ & 05:02:58.1 & $+25: 16: 26.6$ & 177.725 & -9.913 & 5.77 & $1.2 \times 1.1$ & 2.7 & $1.645 \pm 0.009$ & $8.996 \pm 0.015$ \\
\hline 3C 138 & $05: 21: 010.0$ & $+16: 38: 22.1$ & 187.405 & -11.343 & 8.60 & $14.5 \times 5.1$ & 1.0 & $1.115 \pm 0.003$ & $5.949 \pm 0.005$ \\
\hline PKS 0531 & $05: 34: 44.5$ & $+19: 27: 21.4$ & 186.762 & -7.108 & 7.02 & $1.3 \times 1.1$ & 0.5 & $0.535 \pm 0.002$ & $3.313 \pm 0.003$ \\
\hline $3 C 147$ & $05: 42: 36.1$ & $+49: 51: 07.2$ & 161.686 & 10.298 & 22.88 & $4.4 \times 3.8$ & 0.5 & $0.796 \pm 0.001$ & $5.004 \pm 0.002$ \\
\hline $3 \mathrm{C} 154$ & $06: 13: 49.0$ & $+26: 04: 36.7$ & 185.592 & 4.003 & 5.00 & $13.7 \times 12.8$ & 0.7 & $1.704 \pm 0.004$ & $14.759 \pm 0.006$ \\
\hline PKS 0742 & $07: 45: 33.1$ & $+10: 11: 12.7$ & 209.797 & 16.592 & 3.51 & $1.9 \times 1.4$ & 0.6 & $0.011 \pm 0.001$ & $0.028 \pm 0.003$ \\
\hline $3 \mathrm{C} 225 \mathrm{~A}$ & $09: 42: 15.3$ & $+13: 45: 51.3$ & 220.010 & 44.008 & 3.34 & $4.5 \times 1.6$ & 1.2 & $0.828 \pm 0.002$ & $1.487 \pm 0.005$ \\
\hline $3 \mathrm{C} 225 \mathrm{~B}$ & $09: 42: 15.6$ & $+13: 45: 49.3$ & 220.011 & 44.009 & 3.34 & $4.5 \times 1.6$ & 2.3 & $0.791 \pm 0.004$ & $1.434 \pm 0.009$ \\
\hline $3 \mathrm{C} 236$ & 10:06:01.8 & $+34: 54: 10.4$ & 190.065 & 53.980 & 3.24 & $4.8 \times 1.8$ & 0.6 & $0.003 \pm 0.001$ & $0.001 \pm 0.003$ \\
\hline 3C 237 & 10:08:00.0 & $+07: 30: 16.6$ & 232.117 & 46.627 & 6.52 & $6.5 \times 4.4$ & 1.0 & $0.410 \pm 0.001$ & $0.624 \pm 0.004$ \\
\hline $3 \mathrm{C} 245 \mathrm{~A}$ & $10: 42: 44.6$ & $+12: 03: 31.3$ & 233.124 & 56.300 & 3.31 & $1.6 \times 1.4$ & 1.3 & $0.016 \pm 0.002$ & $0.051 \pm 0.005$ \\
\hline $3 \mathrm{C} 245 \mathrm{~B}$ & $10: 42: 44.3$ & $+12: 03: 31.6$ & 233.123 & 56.299 & 3.31 & $1.6 \times 1.4$ & 4.2 & $0.024 \pm 0.006$ & $0.028 \pm 0.015$ \\
\hline $1055+018$ & $10: 58: 29.6$ & $+01: 33: 58.8$ & 251.511 & 52.774 & 3.22 & $0.1 \times 0.0$ & 0.9 & $0.008 \pm 0.001$ & $0.034 \pm 0.004$ \\
\hline 3C 263.1 & $11: 43: 25.1$ & $+22: 06: 56.1$ & 227.201 & 73.766 & 3.13 & $7.5 \times 4.4$ & 0.7 & $0.020 \pm 0.001$ & $0.056 \pm 0.004$ \\
\hline $3 \mathrm{C} 273$ & $12: 29: 06.1$ & $+02: 03: 08.6$ & 289.945 & 64.359 & 54.99 & $7.8 \times 4.2$ & 0.4 & $0.026 \pm 0.001$ & $0.086 \pm 0.002$ \\
\hline 4C 32.44 & $13: 26: 16.5$ & $+31: 54: 09.5$ & 67.234 & 81.048 & 4.86 & $2.8 \times 1.2$ & 0.7 & $0.020 \pm 0.001$ & $0.062 \pm 0.003$ \\
\hline 4C 25.43 & $13: 30: 37.7$ & $+25: 09: 11.0$ & 22.468 & 80.988 & 7.05 & $2.8 \times 1.2$ & 1.1 & $0.004 \pm 0.001$ & $0.029 \pm 0.004$ \\
\hline $3 C 286$ & 13:31:08.3 & $+30: 30: 33.0$ & 56.524 & 80.675 & 14.90 & $5.2 \times 3.3$ & 0.4 & $0.007 \pm 0.001$ & $0.065 \pm 0.002$ \\
\hline 4C 12.50 & $13: 47: 33.4$ & $+12: 17: 24.2$ & 347.223 & 70.172 & 5.40 & $4.3 \times 1.5$ & 0.9 & $0.091 \pm 0.002$ & $0.288 \pm 0.004$ \\
\hline $3 C 298$ & $14: 19: 08.2$ & $+06: 28: 34.8$ & 352.160 & 60.666 & 6.10 & $2.4 \times 1.4$ & 0.6 & $0.020 \pm 0.001$ & $0.077 \pm 0.003$ \\
\hline UGC 09799 & $15: 16: 44.5$ & $+07: 01: 17.8$ & 9.417 & 50.120 & 5.50 & $1.7 \times 1.3$ & 6.9 & $0.066 \pm 0.010$ & $0.135 \pm 0.024$ \\
\hline 4C 04.51 & $15: 21: 14.4$ & $+04: 30: 22.0$ & 7.292 & 47.747 & 3.93 & $9.4 \times 3.8$ & 0.8 & $0.068 \pm 0.001$ & $0.325 \pm 0.003$ \\
\hline $3 \mathrm{C} 327.1 \mathrm{~A}$ & $16: 04: 44.9$ & $+01: 17: 52.8$ & 12.181 & 37.006 & 4.08 & $3.5 \times 2.4$ & 3.2 & $0.505 \pm 0.008$ & $2.259 \pm 0.013$ \\
\hline $3 \mathrm{C} 327.1 \mathrm{~B}$ & $16: 04: 45.6$ & $+01: 17: 47.6$ & 12.182 & 37.003 & 4.08 & $3.5 \times 2.4$ & 3.0 & $0.448 \pm 0.007$ & $2.135 \pm 0.012$ \\
\hline PKS 1607 & 16:09:13.3 & $+26: 41: 29.0$ & 44.171 & 46.203 & 4.91 & $0.9 \times 0.4$ & 0.6 & $0.177 \pm 0.001$ & $0.924 \pm 0.003$ \\
\hline $\mathrm{J} 1613$ & $16: 13: 41.1$ & $+34: 12: 47.9$ & 55.151 & 46.379 & 4.02 & $4.1 \times 3.5$ & 1.0 & $0.005 \pm 0.001$ & $0.001 \pm 0.004$ \\
\hline $3 C 345$ & $16: 42: 58.8$ & $+39: 48: 37.0$ & 63.455 & 40.949 & 7.10 & $3.4 \times 1.5$ & 0.9 & $0.008 \pm 0.001$ & $0.007 \pm 0.004$ \\
\hline $3 C 346$ & $16: 43: 48.6$ & $+17: 15: 49.3$ & 35.332 & 35.769 & 3.66 & $1.0 \times 0.7$ & 2.0 & $0.288 \pm 0.004$ & $1.160 \pm 0.008$ \\
\hline $3 C 390$ & $18: 45: 37.6$ & $+09: 53: 45.0$ & 41.112 & 5.773 & 4.51 & $3.4 \times 2.8$ & 1.2 & $0.157 \pm 0.003$ & $2.649 \pm 0.007$ \\
\hline 4C 33.48 & $19: 24: 17.5$ & $+33: 29: 29.7$ & 66.389 & 8.371 & 3.77 & $4.8 \times 1.7$ & 2.9 & $0.408 \pm 0.006$ & $2.434 \pm 0.013$ \\
\hline $3 \mathrm{C} 409 \mathrm{~A}$ & $20: 14: 27.5$ & $+23: 34: 55.4$ & 63.398 & -6.121 & 13.68 & $1.6 \times 1.4$ & 1.4 & $1.190 \pm 0.005$ & $8.701 \pm 0.008$ \\
\hline $3 \mathrm{C} 409 \mathrm{~B}$ & $20: 14: 27.7$ & $+23: 34: 50.2$ & 63.398 & -6.122 & 13.68 & $1.6 \times 1.4$ & 1.3 & $1.303 \pm 0.004$ & $8.502 \pm 0.007$ \\
\hline $3 \mathrm{C} 410 \mathrm{~A}$ & 20:20:06.6 & $+29: 42: 14.8$ & 69.212 & -3.769 & 2.88 & $3.2 \times 1.7$ & 1.4 & $3.501 \pm 0.007$ & $17.744 \pm 0.010$ \\
\hline $3 \mathrm{C} 410 \mathrm{~B}$ & 20:20:06.7 & $+29: 42: 09.6$ & 69.211 & -3.770 & 6.39 & $3.2 \times 1.7$ & 2.0 & $3.146 \pm 0.009$ & $16.993 \pm 0.014$ \\
\hline B2050 & $20: 52: 52.1$ & $+36: 35: 35.3$ & 78.858 & -5.124 & 5.14 & $4.3 \times 2.0$ & 0.8 & $0.331 \pm 0.002$ & $2.653 \pm 0.004$ \\
\hline $3 \mathrm{C} 433$ & $21: 23: 44.6$ & $+25: 04: 02.2$ & 74.475 & -17.697 & 10.33 & $9.7 \times 5.7$ & 3.0 & $0.467 \pm 0.007$ & $1.882 \pm 0.012$ \\
\hline PKS 2127 & $21: 30: 32.9$ & $+05: 02: 17.5$ & 58.652 & -31.815 & 4.10 & $4.0 \times 1.7$ & 0.7 & $0.128 \pm 0.001$ & $0.556 \pm 0.003$ \\
\hline $\mathrm{J} 2136$ & $21: 36: 38.6$ & $+00: 41: 54.2$ & 55.473 & -35.578 & 3.47 & $8.3 \times 5.0$ & 1.2 & $0.143 \pm 0.002$ & $0.882 \pm 0.005$ \\
\hline $\mathrm{J} 2232$ & $22: 32: 36.4$ & $+11: 43: 50.9$ & 77.438 & -38.582 & 7.20 & $5.2 \times 4.3$ & 1.0 & $0.156 \pm 0.002$ & $1.052 \pm 0.004$ \\
\hline $3 C 454.3$ & 22:53:58.0 & $+16: 08: 52.4$ & 86.112 & -38.185 & 12.66 & $2.2 \times 1.5$ & 1.1 & $0.317 \pm 0.002$ & $1.810 \pm 0.005$ \\
\hline $3 \mathrm{C} 459$ & $23: 16: 35.2$ & $+04: 05: 18.1$ & 83.040 & -51.285 & 4.68 & $5.7 \times 4.5$ & 0.9 & $0.142 \pm 0.002$ & $1.146 \pm 0.004$ \\
\hline \multicolumn{10}{|c|}{ Sources rejected following preliminary observations as overly resolved (res) or saturated (sat) } \\
\hline J0407 (res) & 04:07:25.5 & $+03: 40: 47.3$ & 187.651 & -33.604 & 3.27 & $\ldots$ & $\ldots$ & $\ldots$ & $\cdots$ \\
\hline J0534 (res) & $05: 34: 34.9$ & $+22: 02: 07.2$ & 184.591 & -5.759 & 13.81 & $\ldots$ & $\ldots$ & $\ldots$ & $\cdots$ \\
\hline J1651 (res) & 16:51:03.9 & $+04: 59: 41.9$ & 23.039 & 28.967 & 11.2 & $\ldots$ & $\ldots$ & $\ldots$ & $\ldots$ \\
\hline PKS 1944 (sat) & $19: 46: 47.9$ & $+25: 12: 45.0$ & 61.472 & 0.096 & 4.9 & $\ldots$ & $\ldots$ & $\ldots$ & $\ldots$ \\
\hline J2021 (sat) & $20: 21: 38.7$ & $+37: 31: 10.1$ & 75.833 & 0.402 & 6.6 & $\ldots$ & $\ldots$ & $\ldots$ & $\ldots$ \\
\hline
\end{tabular}

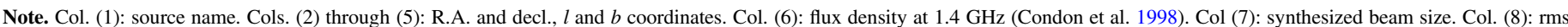

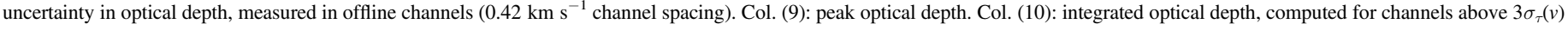
(see Section 2.3). 
available: EBHIS (10". 8 resolution at $21 \mathrm{~cm}$; Winkel et al. 2016).

In Section 2.4 of M15, we described our treatment of $T_{B \text {,exp }}(v)$ spectra from Arecibo. For this work, to estimate the beam efficiency factor for converting antenna temperature to brightness temperature, we compared the integrated antenna temperatures to those derived from averaging brightness temperature spectra from the Galactic Arecibo L-band Feed Array (GALFA-H I; Peek et al. 2011, 2018) survey, which is flux-calibrated based on $\mathrm{LAB}$, in annuli of radius 2 pixels (16 pixels) around each target pixel. From this comparison, we derived a new beam efficiency correction factor, equal to 0.94 , which we applied to the full data set and which ensures that our $T_{B, \exp }(v)$ spectra are consistent with previous surveys (i.e., LAB, EBHIS).

Furthermore, we note that the Arecibo H I emission spectra in this work have not been corrected for radiation entering the main telescope beam from higher order side lobes, an effect known as "stray radiation." Unlike single-dish radio telescopes whose beam shapes can be accurately modeled to remove this effect (e.g., Dwingeloo, Effelsberg, Parkes; Hartmann \& Burton 1997; Kalberla et al. 2005; McClure-Griffiths et al. 2009; Kalberla \& Haud 2015), Arecibo has a very complex beam structure that varies with azimuth and elevation, and therefore stray radiation is extremely difficult to remove. Comparing $21 \mathrm{~cm}$ emission from the GALFA-HI survey with the stray-corrected LAB survey, stray radiation likely contributes $\sim 500 \mathrm{mK}$ over $\sim 50 \mathrm{~km} \mathrm{~s}^{-1}$ to the observed H I brightness temperature (Peek et al. 2011). Considering this effect, we are explicitly careful to not overfit our $21 \mathrm{~cm}$ profiles from Arecibo and emphasize that stray radiation does not affect $21 \mathrm{~cm}$ absorption from the VLA.

\subsection{Uncertainty Arrays}

The uncertainty in each spectral channel depends on the system temperature, which can be significantly increased by strong brightness temperature at Galactic velocities. To determine the frequency-dependent uncertainty arrays for each LOS, we follow the methods described in Section 3.2 of M15, which were derived following Roy et al. (2013a). In summary, for each LOS, the uncertainty array in absorption is a combination of on-source noise $\left(\sigma_{\text {on }}(v)\right)$ and off-source noise from the frequency-switched bandpass solution $\left(\sigma_{\mathrm{BP}}\right)$. The on-source noise is computed by scaling the rms uncertainty in $\exp -\tau(v)$ (i.e., our measured absorption quantity) by $\left(T_{B, \mathrm{LAB}}(v)+T_{\mathrm{sys}, \mathrm{VLA}}\right) / T_{\mathrm{sys}, \mathrm{VLA}}$, where $T_{B, \mathrm{LAB}}(v)$ is the brightness temperature computed from adjacent pixels to each target source from the LAB survey, whose telescope at Dwingeloo is of similar size to a VLA antenna, with an assumed system temperature at the VLA of $T_{\mathrm{sys}, \mathrm{VLA}}=25 \mathrm{~K}$. The uncertainty in $\exp -\tau(v)$ is then computed by solving $\sigma_{\exp -\tau}(v)^{2}=\sigma_{\text {on }}(v)^{2}+$ $\sigma_{\mathrm{BP}}^{2}$. From this, we solve for the uncertainty in $\tau(v)$ (i.e., $\sigma_{\tau}(v)$ ) for subsequent spectral analysis. For each emission spectrum, the uncertainty array $\left(\sigma_{T_{B}}(v)\right)$ is estimated by scaling the rms noise in $T_{B, \exp }(v)$ computed from offline channels by $\left(T_{B, \exp }(v)+T_{\text {sys,em }}\right) / T_{\text {sys,em }}$ for an assumed system temperature of $T_{\mathrm{sys}, \mathrm{em}}=30 \mathrm{~K}$.

\subsection{Line-of-sight Properties}

In Table 1, we list parameters of the 21-SPONGE VLA spectra. First, we include the peak optical depth $\left(\tau_{\text {peak }}\right)$ along the LOS, with uncertainty equal to the value of the $\sigma_{\tau}(v)$ at the velocity of $\tau_{\text {peak }}$. We find a median $\tau_{\text {peak }}=0.32$ and mean $\tau_{\text {peak }}=0.61$. We observe $\tau_{\text {peak }} \geqslant 3$ in only two cases (3C 410A and 3C 410B). Our sources lie generally at high Galactic latitude to avoid the strong velocity crowding associated with the Galactic plane, and therefore the generally small $\tau_{\text {peak }}$ is consistent with expectations. However, we note that $13 / 57$ LOSs lie at low Galactic latitude $(|b|<10)$, providing a comparison sample for the high-latitude population. We also list the integrated optical depth $\left(\int \tau d v\right)$, with uncertainties computed by adding the uncertainty in each spectral channel in quadrature.

\subsection{Synthetic H I Spectra}

To consider the performance of our analysis methods, we will compare the 21-SPONGE spectral line pairs with a sample of synthetic $21 \mathrm{~cm}$ spectral line pairs from KOK14. These synthetic spectra were constructed from the 3D hydrodynamical simulation of KOK13, which includes time-varying heating and cooling of interstellar gas, momentum feedback from supernovae, self-gravity, differential rotation, and external gravity from dark matter and stars. From this simulation, KOK14 selected $10^{4}$ randomly distributed mock sight lines at $|b|>4$.9 within the simulated volume and extracted the number density $(n)$, temperature (kinetic, $T_{k}$, and spin, $T_{s}$ ), and velocity $(v)$ as a function of distance along the LOS. Using analytic radiative transfer and simple line excitation considerations, KOK14 constructed synthetic $21 \mathrm{~cm}$ brightness temperature $\left(T_{B}\right)$ and optical depth $(\tau)$ spectra from each LOS. We refer the reader to Section 2.3 of KOK14 for details of synthetic spectra construction.

In Murray et al. (2017), we found that the implementation of the WF effect has a significant effect on the line widths of the KOK14 synthetic spectral lines and the resulting WNM spin temperature distribution. KOK14 constructed three sets of synthetic $21 \mathrm{~cm}$ data for different WF prescriptions: no WF, constant WF, and maximum WF (i.e., $T_{s}=T_{k}$ ). We found that the constant WF case, wherein the Ly $\alpha$ radiation field density was fixed at $10^{-6}$ photons $\mathrm{cm}^{-3}$, resulted in a narrow spin temperature distribution $\left(T_{s} \sim 4000 \mathrm{~K}\right.$ for $\left.T_{k}>4000 \mathrm{~K}\right)$ for the WNM (cf. Figure 2 of KOK14). Via comparison with 21-SPONGE spectra, we found that KOK14 spectra-whether with no WF or with constant WF-feature a significant population of large-amplitude, wide absorption components not observed in 21-SPONGE yet well above our sensitivity limits (cf. Figure 11 of Murray et al. 2017). Furthermore, these components correspond to WNM properties not observed by 21-SPONGE (i.e., $T_{s} \sim 3000-4000 \mathrm{~K}$ ). From this comparison, we concluded that a more sophisticated treatment of the WF effect is likely necessary to produce realistic synthetic spectral lines from future simulations.

For this study, we select the maximum WF synthetic data set from KOK14. Using an analysis similar to that of Murray et al. (2017), we determined that the maximum WF dataset features spectral components whose line widths agree best with those detected in 21-SPONGE and therefore maximizes consistency between observed and synthetic data sets. To build the synthetic data set, we select spectra without saturated $(\tau \geqslant 3)$ 
absorption, for a final catalog of $9355 \mathrm{HI}$ spectral pairs. To simulate the same observational properties of 21-SPONGE spectral line pairs, we add Gaussian noise to each synthetic $21 \mathrm{~cm}$ spectrum (rms $\sigma_{\tau}=10^{-3}$ for absorption, rms $\sigma_{T_{B}}=0.2 \mathrm{~K}$ for emission) as done in Murray et al. (2017).

\section{Analysis}

To derive the physical properties of individual H I structures along each LOS, we decompose all $\mathrm{H}$ I emission and absorption spectral line pairs into Gaussian functions. In the following section, we describe our method for autonomously decomposing $21 \mathrm{~cm}$ spectra.

\subsection{Gaussian Decomposition}

We begin by decomposing the VLA H I absorption spectra uniformly using the Autonomous Gaussian Decomposition algorithm (AGD; Lindner et al. 2015) via its open-source Python implementation, GaussPy. ${ }^{10}$ AGD implements derivative spectroscopy and supervised machine learning to produce efficient, reproducible guesses for the basic parameters of Gaussian functions, including the number of components, their amplitudes, positions, and widths. Following the method described in Lindner et al. (2015) and employed in Murray et al. (2017), we train the algorithm using a synthetic absorption-line data set constructed from spectral line parameters from HT03. From the training process, we determine optimal values of the two-phase smoothing parameters, $\alpha_{1}=1.12$ and $\alpha_{2}=2.75$, required by AGD to compute spectral line parameter guesses. We then decompose the 21-SPONGE absorption lines using these values with an imposed minimum signal-to-noise ratio of $\mathrm{S} / \mathrm{N}=3$. To avoid aliasing narrow components, we first resample the spectra to a velocity resolution of $0.1 \mathrm{~km} \mathrm{~s}^{-1}$ (Lindner et al. 2015). As shown in Lindner et al. (2015) and Murray et al. (2017), the resulting parameters of the decomposition are statistically indistinguishable from those found in the by-hand analysis of 21-SPONGE sources. Although no Gaussian decomposition represents a unique solution, we emphasize the benefits of the AGD: to eliminate subjective biases of human-derived guesses and to ensure that the decomposition results are completely reproducible.

After decomposing each $\mathrm{H}$ I absorption spectrum using AGD into $N$ components, we produce a model for the optical depth along the LOS:

$$
\tau(v)_{\mathrm{AGD}}=\sum_{n=0}^{N-1} \tau_{0, n} \cdot e^{-4 \ln 2\left(v-v_{0, n}\right)^{2} / \Delta v_{n}^{2}}
$$

where $\left(\tau_{0, n}, v_{0, n}, \Delta v_{n}\right)$ are the amplitude, mean velocity, and FWHM of the $n$th component.

To fit these components to the expected brightness temperature along the $\operatorname{LOS}\left(T_{B, \exp }(v)\right)$, we assume a twocomponent H I medium, wherein some clouds contribute both opacity and brightness temperature (i.e., detected in emission and absorption), and some clouds are dominated by the WNM and contribute only brightness temperature to the LOS (e.g., Mebold et al. 1997; Dickey et al. 2000, HT03, M15). For each

\footnotetext{
${ }^{10}$ GaussPy; https://github.com/gausspy/gausspy.
}

LOS, we solve,

$$
T_{B, \exp , \mathrm{AGD}}(v)=T_{B, \mathrm{abs}, \mathrm{AGD}}(v)+T_{B, \mathrm{em}, \mathrm{AGD}}(v) .
$$

To determine the contributions of absorption-detected and emission-only components to $T_{B, \exp }(v)$, we implement a new method to fit all components to $T_{B, \exp }(v)$ using AGD, based on the strategy described in HT03. We note that whereas the original HT03 method involved fitting components by eye, our new method is autonomous. The method involves the following steps:

1. Fit all $N$ components from $\tau(v)$ to $T_{B, \exp }(v)$ via a leastsquares fit. The mean velocities and widths are allowed to vary by $\pm 10 \%$ to simulate small random fluctuations and their amplitudes are constrained so that $0<T_{B, n}=T_{s, n}$. $\left(1-e^{-\tau_{0, n}}\right)$ and $T_{s, n} \leqslant T_{k, \max , n}=21.866 \cdot \Delta v_{n}^{2}$ to produce realistic spin temperatures.

2. Subtract the best-fit model in step (1) from $T_{B, \exp }(v)$ to produce a residual emission spectrum, which contains only emission not detected in absorption.

3. Apply GaussPy to fit $K$ new components to the residual emission spectrum from (2), using the trained one-phase value of $\alpha=3.75$ and $\mathrm{S} / \mathrm{N} \geqslant 5$ from previous analysis of HT03 emission spectra (Murray et al. 2017). The S/N requirement is stricter in the fit to emission than the initial absorption fit so that we avoid overfitting the emission residuals in the presence of stray radiation. We also remove any component guesses whose mean velocities agree with previously detected absorption components within 1 spectral channel (i.e., $0.42 \mathrm{~km} \mathrm{~s}^{-1}$ ) so as not to spuriously overfit $T_{B, \exp }(v)$ (this occurs only in the presence of strong residuals following the subtraction of absorption components in complex LOS).

4. Combine the $N+K$ Gaussian components from steps (1) and (3) and execute a final least-squares fit to $T_{B, \exp }(v)$. In this final fit, we allow all mean velocities and widths to vary by $10 \%$, and constrain all amplitudes such that $T_{B \text {,exp }}>0$. In this step, initial estimates of $T_{s}$ for the $N$ absorption components and the Gaussian parameters of the $K$ emission-only components are computed.

Given a final list of $N+K$ Gaussian components fitted to $T_{B \text {,exp }}(v)$ from the procedure described above, we solve Equation (2) for all possible orderings of the $N$ absorption components along the LOS and for varying absorption properties of the $K$ emission-only components, following HT03. In detail, we solve:

$$
T_{B, \mathrm{abs}, \mathrm{AGD}}(v)=\sum_{n=0}^{N-1} T_{s, n}\left(1-e^{-\tau_{n}(v)}\right) e^{-\sum_{m=0}^{M} \tau_{m}(v)},
$$

where the subscript $m$ refers to all components which lie in front of the $n$th component, and

$$
\begin{aligned}
T_{B, \mathrm{em}, \mathrm{AGD}}(v)= & \sum_{k=0}^{K-1}\left[\mathscr{F}_{k}+\left(1-\mathscr{F}_{k}\right) e^{-\tau(v)}\right] \\
& \cdot T_{0, k} e^{\frac{-4 \ln 2\left(v-v_{0, k}\right)^{2}}{\Delta v_{k}^{2}}},
\end{aligned}
$$

where $\left(T_{0, k}, v_{0, k}, \Delta v_{k}\right)$ are the amplitude, mean velocity, and FWHM of the $k$ th component fitted only in emission, and $\mathscr{F}_{k}$ is the fraction of each component lying in front of all absorption components. Previous analysis has shown that $\mathscr{F}$ is highly 

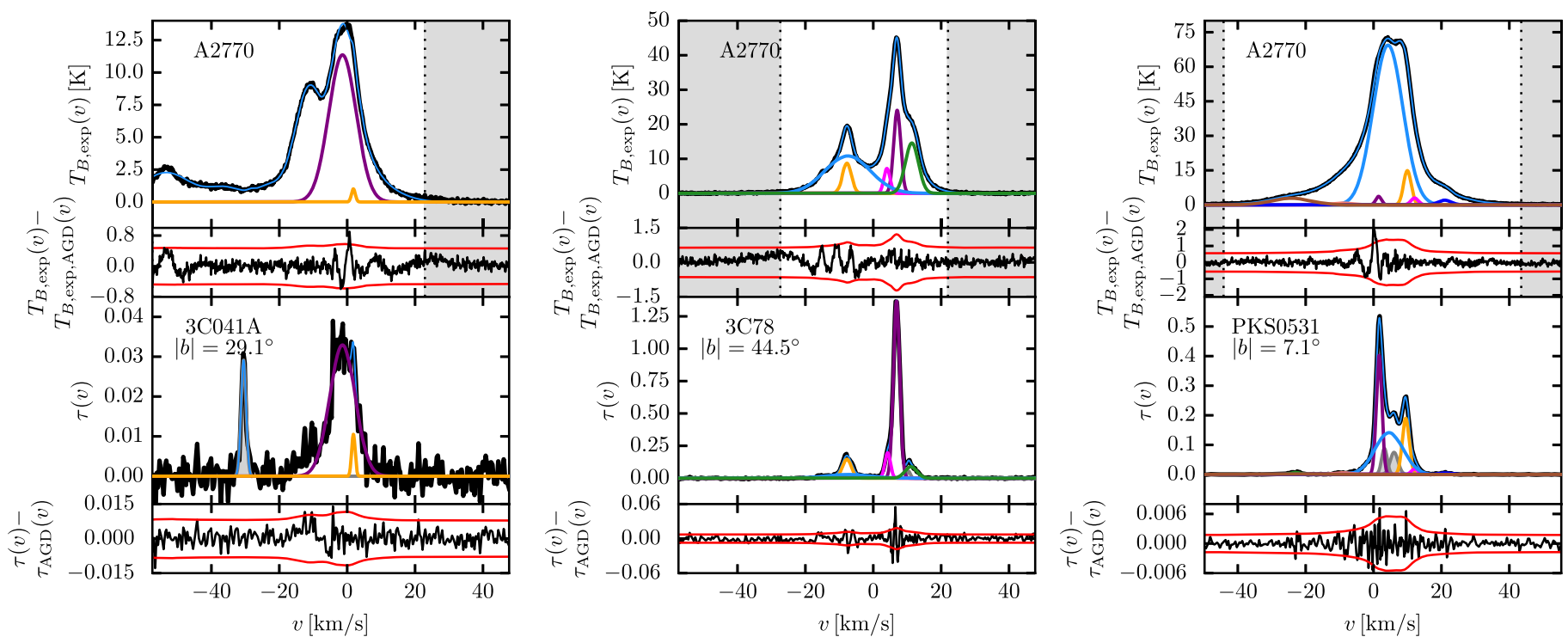

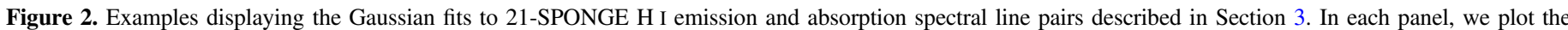

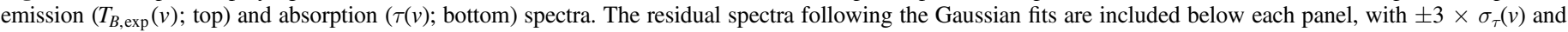

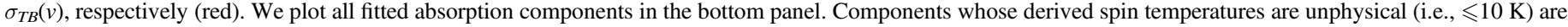

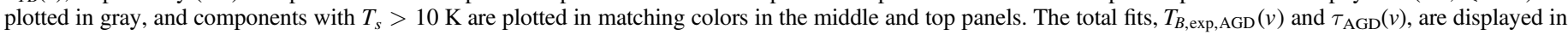

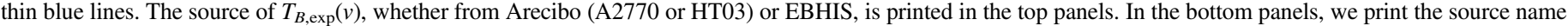

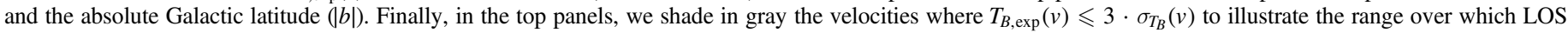

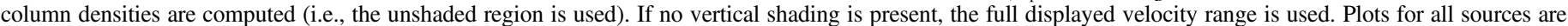
included in the Appendix.

uncertain (e.g., HT03; Stanimirović et al. 2014; M15) and yet it has a significant effect on the derived spin temperatures. Therefore, following HT03, we allow $\mathscr{F}_{k}$ to have one of three values, $\mathscr{F}_{k}=0.0,0.5$, or 1.0 for all $K$ emission-only components.

Furthermore, the order of the absorption components along the LOS will only affect $T_{B \text {,abs }}(v)$ in the cases of components that overlap significantly in velocity (HT03). For each LOS, there are a maximum of $N$ ! possible orderings, but we select only the unique orderings corresponding to components that overlap in area by more than $3 \sigma_{\tau} d v$. Therefore, there are a total of $3 \times N$ ! possible iterations for the final fit to Equation (2), but in practice there are many fewer for each LOS.

We determine a final estimate for the spin temperature of each absorption-detected component by computing the weighted mean and standard deviation over all ordering trials (cf. Equations (21a) and (21b) of HT03). We fit a total of 280 absorption components and 278 emission-only components to the 57 LOSs. Figure 2 displays three examples of the fitting process described above. Similar plots for all 57 LOSs are included in the Appendix, Figure Set 13.

\subsection{Synthetic H I Decomposition}

We use the same methodology described above to fit 9355 synthetic H I absorption and emission spectral line pairs from KOK14 with maximum WF. In contrast to the analysis of Murray et al. (2017), wherein we presented a method for matching Gaussian spectral lines to "true" gas structures along the LOS, the new method presented here improves the statistics of components for which we can derive $T_{s}$ and $N(\mathrm{H} \mathrm{I})$. The method described in Murray et al. (2017) selected only those components with unambiguous signatures in $\tau(v), T_{B \text {,exp }}(v)$, and $n / T_{s}$, and therefore resulted in fewer detected components per
LOS. Here we fit a total of 12,715 components to the 9355 synthetic absorption lines.

\section{Fitting Results}

\subsection{Properties of Fitted Components}

For each absorption-detected component, we compute the maximum kinetic temperature $\left(T_{k, \max }\right)$ via

$$
T_{k, \max }=\frac{m_{\mathrm{H}}}{8 k_{\mathrm{B}} \ln 2} \Delta v_{0}^{2}=21.866 \cdot \Delta v_{0}^{2}
$$

for hydrogen mass $m_{\mathrm{H}}$ and Boltzmann's constant $k_{\mathrm{B}}$ (Draine 2011).

Next, we compute the H I column density per absorption component, given by

$$
N(\mathrm{H} \mathrm{I})_{\mathrm{abs}}=C_{0} \int \tau T_{s} d v=1.064 \cdot C_{0} \cdot \tau_{0} \cdot \Delta v_{0} \cdot T_{s},
$$

where $C_{0}=1.823 \times 10^{18} \mathrm{~cm}^{-2} /\left(\mathrm{km} \mathrm{s}^{-1} \mathrm{~K}\right), \Delta v_{0}$ is measured in $\mathrm{km} \mathrm{s}^{-1}$, and 1.064 converts the product to the area under a Gaussian function with the given height and width.

Figure 3 displays cumulative distribution functions (CDFs) of the fitted parameters for all absorption-detected components with physically reasonable values of $T_{s}$, defined as $T_{s}>10 \mathrm{~K}$. This limit for "reasonable" temperatures is defined conservatively based on the estimated contribution of the cosmic microwave and Galactic synchrotron backgrounds at the locations of our sources, which we found to vary between 2.76 and $2.85 \mathrm{~K}$ for sources from the first half of the 21-SPONGE survey (M15), plus an estimated minimum $T_{k}$ of the CNM ( $\sim 7 \mathrm{~K}$; Wolfire et al. 2003). Out of 280 components, 222 have $T_{s}>10 \mathrm{~K}$.

In the top row of Figure 3, we display parameters from the best fit to $\tau(v)$, including optical depth amplitude $\left(\tau_{0}\right)$, FHWM $\left(\Delta v_{0}\right)$, and absolute mean velocity $\left(\left|v_{0}\right|\right)$. In the bottom row, we 

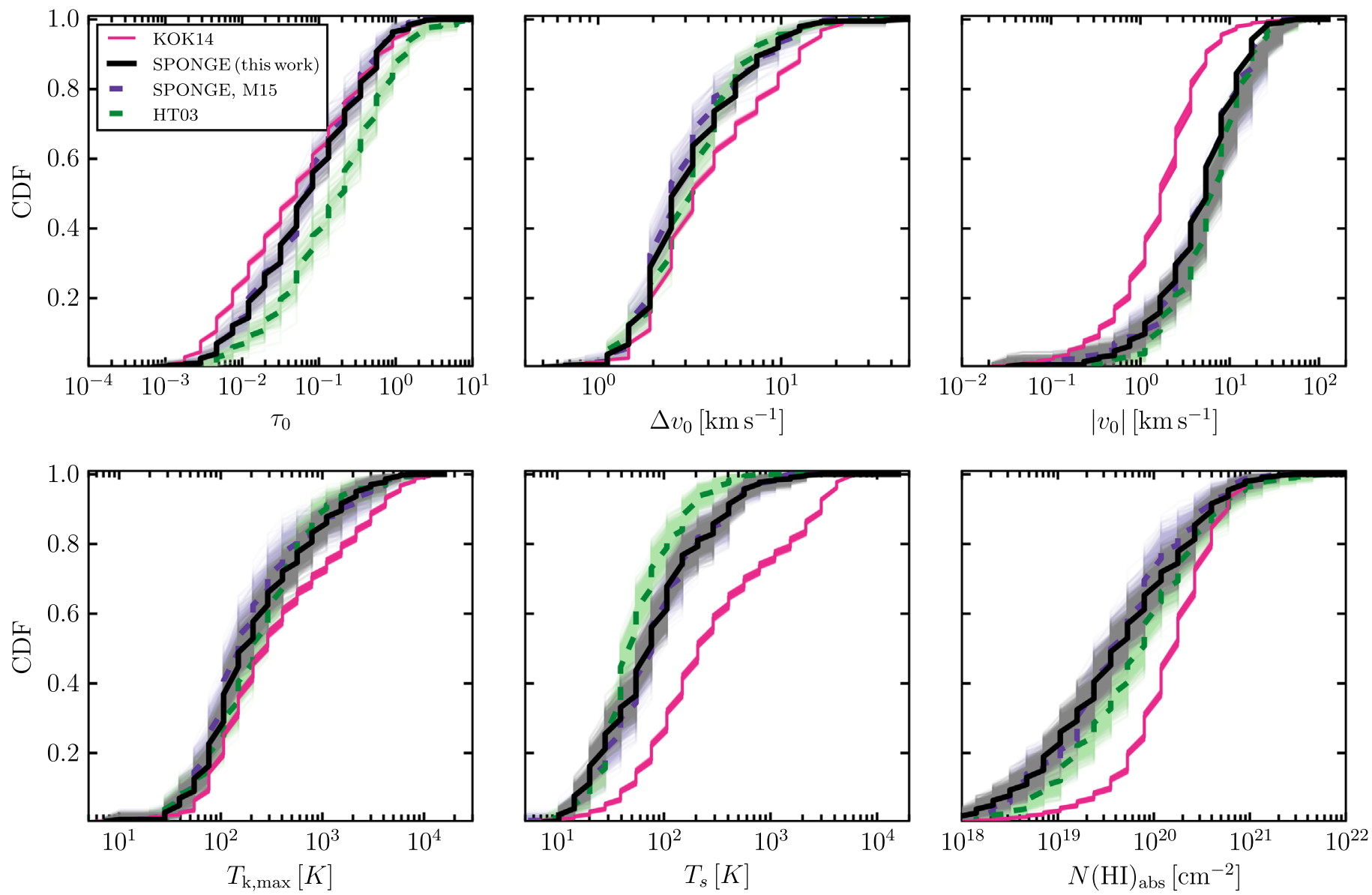

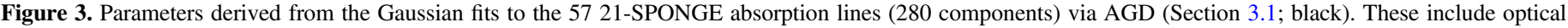

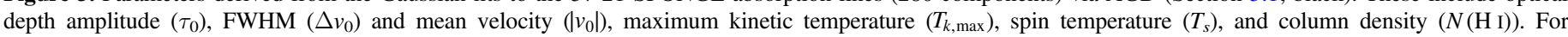

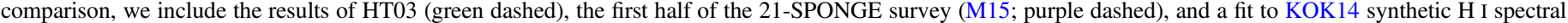
line pairs using the same methodology (pink).

include derived physical properties. In all panels of Figure 3, we bootstrap each sample over 100 trials and include the resampled CDFs to illustrate the effect of outliers on the distributions. For comparison, we include the results of HT03, M15, and a reprocessing of the synthetic H I spectral line pair database from KOK14 with maximum WF (Section 3.2). The parameters for all 21-SPONGE sources are listed in Table 5 in the Appendix. The uncertainties for all parameters are computed as part of the least-squares AGD fit, except for the uncertainty in $T_{s}$ (and, subsequently $N(\mathrm{HI})_{\mathrm{abs}}$ ), which is computed following the iterations over LOS component ordering. We set the minimum uncertainty in the optical depth amplitudes equal to $\sigma_{\tau}(v)$ at the position of each component, and the minimum uncertainty in the mean velocity and FWHM is equal to $0.1 \mathrm{~km} \mathrm{~s}^{-1}$. We note that for one absorption component toward 3C 111A, the uncertainties from the AGD fit are extremely large $(\gg 1000)$ - this component was not recovered in the fit to $T_{B, \exp }(v)$, and we identify it by setting the uncertainties in its component parameters equal to 99 in Table 5.

In comparison with the previous observations shown in Figure 3, we find that our decomposition results (black) are statistically indistinguishable from the by-hand analysis of the first half of the 21-SPONGE survey (purple dashed; M15 wherein we analyzed a subset of the 21-SPONGE sample presented in this work. We are also generally consistent with
HT03, except in the case of $\tau_{0}$, wherein the superior sensitivity of 21-SPONGE allows us to probe smaller H I optical depths. In addition, we detect a higher $T_{s}$ in the present analysis than found by HT03, which is also attributable to our improved observational sensitivity.

In Figure 3, we find that the observed (SPONGE, HT03) and synthetic (KOK14) $T_{s}$ and $N(\mathrm{H} \mathrm{I})$ distributions are significantly different. We will discuss this further in Section 6.

\subsection{Correspondence between $21 \mathrm{~cm}$ Absorption and Emission}

Overall, most (78\%) of the $N$ fitted components to $\tau(v)$ have corresponding components in the fit to $T_{B, \exp }(v)$. Even when all $\tau(v)$ components are forced to be included in the fit to $T_{B, \exp }(v)$ (e.g., as in the method of HT03, M15), $10 \%$ of components end up with unphysical spin temperatures. Components with $T_{s} \leqslant 10 \mathrm{~K}$ are displayed in gray in Figure Set 13 .

However, the overall fraction of absorption components with corresponding detected emission is generally high: for $22 \%$ of the 57 LOSs, $100 \%$ of the absorption-fitted components correspond to components in the fit to $T_{B, \exp }(v)$, and for $98 \%$ of LOSs, $\geqslant 50 \%$ of absorption components correspond to $T_{B \text { exp }}(v)$ components. The LOSs featuring the lowest fraction of corresponding components between absorption and emission tend to lie at low Galactic latitude, where velocity blending of spectral lines is strongest. We find consistent statistics in the decomposition of KOK14 spectra. We will discuss the 
implications of the observed correspondence between absorption and emission further in Section 6.1.

An important effect in producing absorption components with no corresponding emission components (i.e., components with $T_{s}<10 \mathrm{~K}$ ) is beam mismatch between absorption and emission. In addition, $21 \mathrm{~cm}$ emission profiles are necessarily measured using adjacent LOSs from the absorption profile in order to avoid the background continuum source, which means that the two profiles are not sampling identical populations of H I structures. For emission spectra from Arecibo (e.g., A2770, HT03), $T_{B \text {,exp }}(v)$ is computed on $\sim 4^{\prime}$ scales, and for those from EBHIS, $T_{B, \exp }(v)$ is computed on 10 ". 8 scales, in contrast with sub-arcminute scales for absorption from the VLA. An example of this effect is shown in the case of 3C 041A (Figure 2).The absorption line clearly detected at $v \sim-30 \mathrm{~km} \mathrm{~s}^{-1}$ is not recovered in $T_{B, \exp }(v)$, likely due to a beam mismatch or LOS effect.

We find that varying the decomposition scheme described in Section 3.1 did not have a significant effect on the results or derived physical parameters. Allowing the component parameters to vary by between $1 \%$ and $20 \%$ in step (1) has the largest effect on the number and properties of the fitted emission-only components. For example, the fraction of absorption-detected components recovered in the fit to $T_{B \text {,exp }}(v)$ and their derived $T_{s}$ values do not vary significantly for different allowed variances.

\section{Column Densities}

Following decomposition, we compute and compare different estimates of the total column density along 21-SPONGE LOSs. Given that the majority of our $21 \mathrm{~cm}$ brightness temperatures have not been corrected for stray radiation, we conservatively restrict our analysis of $T_{B, \exp }(v)$ to channels above the uncertainty array for each LOS, specifically: $T_{B, \exp }(v) \geqslant 3 \sigma_{T_{B}}(v)$. In the top panels of each source plot in Figures 2 and 13 we shade the velocity channels which are not used in the column density analysis in light gray. The minimum and maximum velocities satisfying $T_{B, \exp }(v) \geqslant 3 \sigma_{T_{B}}(v)$ are referred to in subsequent discussion as $v_{\mathrm{em}, \min }$ and $v_{\mathrm{em}, \max }$, respectively.

In the absence of optical depth information, it is common in the literature to assume that neutral gas is optically thin so that the LOS column density can be computed via

$$
N(\mathrm{H} \mathrm{I})_{\text {thin }}=C_{0} \int_{v_{\mathrm{em}, \min }}^{v_{\mathrm{em}, \max }} T_{B, \exp }(v) d v,
$$

where $d v$ is measured in $\mathrm{km} \mathrm{s}^{-1}$. Incorporating the optical depth information from 21-SPONGE, assuming that all $\mathrm{HI}$ within each velocity channel is isothermal (e.g., Dickey \& Benson 1982; Chengalur et al. 2013), the column density is given by

$$
N(\mathrm{H} \mathrm{I})_{\text {iso }}=C_{0} \int_{v_{\mathrm{em}, \min }}^{v_{\mathrm{em}, \max }} \frac{\tau(v) T_{B, \exp }(v)}{1-e^{-\tau(v)}} d v .
$$

However, considering significant overlap in the velocity of individual spectral features in Figure Set 13, there may be multiple H I structures with different physical properties within the same radial velocity channels, potentially invalidating the isothermal approximation in Equation (8). Using the results of our spectral decomposition, we compute the "total" H I column density,

$$
\begin{aligned}
N(\mathrm{H} \mathrm{I})_{\mathrm{total}}= & \sum_{n=0}^{N-1} N(\mathrm{H} \mathrm{I})_{\mathrm{abs}, n}+ \\
& +C_{0} \int_{v_{\mathrm{em}, \min }}^{v_{\mathrm{em}, \max }}\left(\sum_{k=0}^{K-1} T_{0, k} e^{\frac{-4 \ln 2\left(v-v_{0, k}\right)^{2}}{\Delta v_{k}^{2}}}\right) d v,
\end{aligned}
$$

where we first sum over the column densities of all $N$ components detected in both absorption and emission $\left(N(\mathrm{HI})_{\text {abs }}\right.$; Equation (6)) and then add the total brightness temperature of the $K$ components detected in emission only for channels above $3 \sigma_{T_{B}}(v)$ (i.e., between $v_{\mathrm{em}, \min }$ and $\left.v_{\mathrm{em}, \max }\right)$ under the optically thin approximation. We restrict the integration of the emission-only components so that $N(\mathrm{HI})_{\text {total }}$ can be compared with $N(\mathrm{HI})_{\text {thin }}$ and $N(\mathrm{HI})_{\text {iso. The }}$ uncertainty in $N(\mathrm{HI})_{\text {total }}$ is computed as the standard deviation over 1000 Monte Carlo trials for each LOS wherein all Gaussian component parameters are drawn from a normal distribution around each parameter (e.g., Heiles \& Troland 2003b; Stanimirović et al. 2014).

In Table 2, we list $N(\mathrm{H} \mathrm{I})_{\text {thin }}, N(\mathrm{H} \mathrm{I})_{\text {iso }}$, and $N(\mathrm{H} \mathrm{I})_{\text {total }}$ for all 57 21-SPONGE LOSs, with uncertainties propagated in quadrature from $\sigma_{T_{B}}(v), \sigma_{\tau}(v)$ for $N(\mathrm{HI})_{\text {thin }}$ and $N(\mathrm{HI})_{\text {iso. }}$. We observe that $N(\mathrm{HI})_{\text {total }}$ and $N(\mathrm{HI})_{\text {iso }}$ are consistent within uncertainties, indicating that our autonomous decomposition method is performing well and recovering the majority of $\tau(v)$ for all LOSs.

To compare these column densities, we explore the effect of optical depth on the LOS column density by estimating the "correction factor" to be applied to $N(\mathrm{HI})_{\text {thin }}$ to account for optical depth effects. Specifically, we compute two versions, one for the isothermal column density, $\mathcal{R}_{\text {iso }}=N(\mathrm{H} \mathrm{I})_{\text {iso }} /$ $N(\mathrm{H} \mathrm{I})_{\text {thin }}$, and one for the "total" column density, $\mathcal{R}_{\text {total }}=$ $N(\mathrm{H} \mathrm{I})_{\text {total }} / N(\mathrm{H} \mathrm{I})_{\text {thin }}$. In Figure 4 , we plot $\mathcal{R}_{\text {iso }}$ and $\mathcal{R}_{\text {total }}$ as a function of $N(\mathrm{HI})_{\text {thin. }}$. For low $\mathrm{HI}$ column densities, $N(\mathrm{H} \mathrm{I})_{\text {thin }} \lesssim 5 \times 10^{20} \mathrm{~cm}^{-2}, \mathcal{R}_{\text {iso }}$ and $\mathcal{R}_{\text {total }}$ are equal to or consistent with unity within uncertainties. The scatter is larger (and uncertainties are higher) in the case of $\mathcal{R}_{\text {total }}$ due to the uncertainty in fitting components between absorption and emission. In particular, the uncertainty in $\mathcal{R}_{\text {total }}$ is only significant at low Galactic latitudes where significant line blending yields large uncertainties in fitted parameters.

In Figure $4, \mathcal{R}_{\text {iso }}$ and $\mathcal{R}_{\text {total }}$ exhibit the same overall behavior as a function of $N(\mathrm{HI})_{\text {thin }}$, which has also been pointed out and discussed as part of a recent study of $\mathrm{HI}$ in the Perseus molecular cloud (Lee et al. 2015) and recent analysis of data from HT03 and the first half of the 21-SPONGE survey (Nguyen et al. 2018). This agreement indicates that our autonomous spectral decomposition method recovers well the velocity structure of $21 \mathrm{~cm}$ absorption and emission for the majority of sources. We will discuss this further in Section 6.1.

In Table 2, we also include the total column density along each LOS in the CNM and UNM phases, $N(\mathrm{HI})_{\mathrm{CNM}}$ and $N(\mathrm{H} \mathrm{I})_{\mathrm{UNM}}$, as the sum of all components with $T_{s, \mathrm{CNM}} \leqslant 250 \mathrm{~K}$ and $250<T_{s \text {,UNM }} \leqslant 1000 \mathrm{~K}$ (defined following predictions from Wolfire et al. 2003 in the solar circle), respectively. We then estimate the fraction of $N(\mathrm{HI})_{\text {total }}$ in each phase, with the assumption that the remaining mass is in the WNM. These mass fraction estimates are uncertain, as both uncertainties in the observations and the AGD-based fit contribute. To estimate the uncertainties in $f_{\mathrm{CNM}}$ and $f_{\mathrm{UNM}}$, we compute the standard deviation of $N(\mathrm{HI})_{\mathrm{CNM}}$ and $N(\mathrm{HI})_{\mathrm{UNM}}$ over the Monte Carlo 
Table 2

Column Densities

\begin{tabular}{|c|c|c|c|c|c|c|c|}
\hline $\begin{array}{l}\text { Source } \\
\text { (name) }\end{array}$ & $\begin{array}{l}N(\mathrm{H} \mathrm{I})_{\text {thin }} \\
\left(10^{20} \mathrm{~cm}^{-2}\right)\end{array}$ & $\begin{array}{c}N(\mathrm{H} \mathrm{I})_{\text {iso }} \\
\left(10^{20} \mathrm{~cm}^{-2}\right)\end{array}$ & $\begin{array}{c}N(\mathrm{H} \mathrm{I})_{\text {total }} \\
\left(10^{20} \mathrm{~cm}^{-2}\right)\end{array}$ & $\begin{array}{c}N(\mathrm{H} \mathrm{I})_{\mathrm{CNM}} \\
\left(10^{20} \mathrm{~cm}^{-2}\right)\end{array}$ & $\begin{array}{c}N(\mathrm{H} \mathrm{I})_{\mathrm{UNM}} \\
\left(10^{20} \mathrm{~cm}^{-2}\right)\end{array}$ & $f_{\mathrm{CNM}}$ & $f_{\mathrm{UNM}}$ \\
\hline J0022 & $2.63 \pm 0.14$ & $2.64 \pm 0.14$ & $2.63 \pm 0.08$ & 0.11 & 0.88 & $0.04 \pm 0.07$ & $0.33 \pm 0.37$ \\
\hline 3C018A & $5.65 \pm 0.02$ & $6.29 \pm 0.03$ & $6.71 \pm 0.24$ & 3.29 & 0.00 & $0.49 \pm 0.21$ & $0.00 \pm 0.28$ \\
\hline $3 \mathrm{C} 018 \mathrm{~B}$ & $5.65 \pm 0.02$ & $6.27 \pm 0.03$ & $6.67 \pm 0.24$ & 3.29 & 0.00 & $0.49 \pm 0.22$ & $0.00 \pm 0.42$ \\
\hline 3C041A & $5.43 \pm 0.23$ & $5.46 \pm 0.23$ & $5.46 \pm 0.11$ & 0.02 & 1.99 & $0.00 \pm 0.10$ & $0.37 \pm 0.51$ \\
\hline $3 \mathrm{C} 041 \mathrm{~B}$ & $5.43 \pm 0.23$ & $5.47 \pm 0.23$ & $5.47 \pm 0.08$ & 0.88 & 0.00 & $0.16 \pm 0.15$ & $0.00 \pm 0.76$ \\
\hline $3 \mathrm{C} 48$ & $4.24 \pm 0.26$ & $4.27 \pm 0.26$ & $4.26 \pm 0.05$ & 0.16 & 1.53 & $0.04 \pm 0.14$ & $0.36 \pm 0.21$ \\
\hline 4C15.05 & $4.33 \pm 0.15$ & $4.42 \pm 0.16$ & $4.42 \pm 0.24$ & 0.39 & 3.11 & $0.09 \pm 0.04$ & $0.70 \pm 0.18$ \\
\hline $3 \mathrm{C} 78$ & $9.76 \pm 0.22$ & $11.44 \pm 0.26$ & $11.37 \pm 0.86$ & 4.43 & 3.17 & $0.39 \pm 0.20$ & $0.28 \pm 0.24$ \\
\hline $4 C 16.09$ & $9.96 \pm 0.16$ & $11.01 \pm 0.17$ & $10.93 \pm 0.28$ & 4.36 & 2.37 & $0.40 \pm 0.24$ & $0.22 \pm 0.08$ \\
\hline $3 \mathrm{C} 111 \mathrm{~A}$ & $24.25 \pm 1.06$ & $28.43 \pm 1.24$ & $28.60 \pm 1.25$ & 19.82 & 0.69 & $0.69 \pm 0.18$ & $0.02 \pm 0.09$ \\
\hline 3C111B & $24.25 \pm 1.06$ & $28.60 \pm 1.24$ & $28.04 \pm 1.78$ & 9.97 & 8.86 & $0.36 \pm 0.22$ & $0.32 \pm 0.09$ \\
\hline $3 \mathrm{C} 111 \mathrm{C}$ & $24.25 \pm 1.06$ & $29.21 \pm 1.27$ & $24.14 \pm 3.17$ & 13.17 & 0.00 & $0.55 \pm 0.22$ & $0.00 \pm 0.19$ \\
\hline $3 \mathrm{C} 120$ & $10.02 \pm 0.03$ & $15.85 \pm 0.04$ & $14.07 \pm 1.09$ & 7.48 & 0.00 & $0.53 \pm 0.24$ & $0.00 \pm 0.08$ \\
\hline $3 \mathrm{C} 123 \mathrm{~A}$ & $15.04 \pm 0.71$ & $19.62 \pm 0.93$ & $19.75 \pm 3.55$ & 5.57 & 5.36 & $0.28 \pm 0.24$ & $0.27 \pm 0.10$ \\
\hline $3 \mathrm{C} 123 \mathrm{~B}$ & $15.04 \pm 0.71$ & $19.51 \pm 0.92$ & $19.68 \pm 3.61$ & 5.73 & 6.07 & $0.29 \pm 0.22$ & $0.31 \pm 0.19$ \\
\hline $3 \mathrm{C} 132$ & $22.83 \pm 0.27$ & $27.15 \pm 0.32$ & $24.95 \pm 2.04$ & 8.51 & 0.00 & $0.34 \pm 0.14$ & $0.00 \pm 0.07$ \\
\hline $3 \mathrm{C} 133$ & $25.11 \pm 0.07$ & $30.07 \pm 0.09$ & $28.80 \pm 2.13$ & 3.01 & 15.93 & $0.10 \pm 0.08$ & $0.55 \pm 0.14$ \\
\hline $3 \mathrm{C} 138$ & $18.55 \pm 0.18$ & $21.32 \pm 0.20$ & $20.82 \pm 2.21$ & 7.16 & 0.89 & $0.34 \pm 0.12$ & $0.04 \pm 0.07$ \\
\hline PKS0531 & $25.13 \pm 0.36$ & $27.10 \pm 0.39$ & $27.15 \pm 1.74$ & 1.61 & 14.65 & $0.06 \pm 0.26$ & $0.54 \pm 0.07$ \\
\hline $3 \mathrm{C} 147$ & $17.26 \pm 0.33$ & $18.71 \pm 0.35$ & $18.43 \pm 1.50$ & 7.14 & 0.00 & $0.39 \pm 0.18$ & $0.00 \pm 0.04$ \\
\hline $3 \mathrm{C} 154$ & $34.11 \pm 0.05$ & $46.48 \pm 0.07$ & $38.87 \pm 10.88$ & 2.26 & 26.60 & $0.06 \pm 0.38$ & $0.68 \pm 0.32$ \\
\hline PKS0742 & $3.13 \pm 0.19$ & $3.13 \pm 0.20$ & $3.11 \pm 0.09$ & 0.09 & 0.00 & $0.03 \pm 0.05$ & $0.00 \pm 0.25$ \\
\hline $3 \mathrm{C} 225 \mathrm{~A}$ & $3.53 \pm 0.03$ & $3.65 \pm 0.03$ & $3.63 \pm 0.10$ & 0.42 & 0.64 & $0.12 \pm 0.08$ & $0.18 \pm 0.40$ \\
\hline $3 \mathrm{C} 225 \mathrm{~B}$ & $3.53 \pm 0.03$ & $3.65 \pm 0.03$ & $3.65 \pm 0.13$ & 0.62 & 1.00 & $0.17 \pm 0.14$ & $0.27 \pm 0.71$ \\
\hline $3 \mathrm{C} 236$ & $0.78 \pm 0.15$ & $0.78 \pm 0.15$ & $0.78 \pm 0.15$ & 0.00 & 0.00 & $0.00 \pm 0.20$ & $0.00 \pm 0.99$ \\
\hline $3 \mathrm{C} 237$ & $2.16 \pm 0.03$ & $2.19 \pm 0.03$ & $2.19 \pm 0.08$ & 0.13 & 0.63 & $0.06 \pm 0.11$ & $0.29 \pm 0.57$ \\
\hline $3 \mathrm{C} 245 \mathrm{~A}$ & $2.19 \pm 0.13$ & $2.20 \pm 0.13$ & $2.19 \pm 0.09$ & 0.00 & 0.40 & $0.00 \pm 0.15$ & $0.18 \pm 0.73$ \\
\hline $3 \mathrm{C} 245 \mathrm{~B}$ & $2.19 \pm 0.13$ & $2.20 \pm 0.13$ & $1.92 \pm 0.13$ & 0.00 & 0.00 & $0.00 \pm 0.45$ & $0.00 \pm 1.00$ \\
\hline $1055+018$ & $2.94 \pm 0.16$ & $2.94 \pm 0.16$ & $2.94 \pm 0.08$ & 0.00 & 0.83 & $0.00 \pm 0.07$ & $0.28 \pm 0.37$ \\
\hline $3 C 263.1$ & $1.81 \pm 0.18$ & $1.81 \pm 0.18$ & $1.81 \pm 0.00$ & 0.03 & 0.00 & $0.02 \pm 0.16$ & $0.00 \pm 0.82$ \\
\hline $3 \mathrm{C} 273$ & $2.11 \pm 0.05$ & $2.11 \pm 0.05$ & $2.10 \pm 0.08$ & 0.02 & 0.29 & $0.01 \pm 0.05$ & $0.14 \pm 0.24$ \\
\hline $4 C 32.44$ & $1.17 \pm 0.14$ & $1.17 \pm 0.14$ & $1.16 \pm 0.01$ & 0.11 & 0.07 & $0.10 \pm 0.14$ & $0.06 \pm 0.69$ \\
\hline $4 \mathrm{C} 25.43$ & $1.07 \pm 0.12$ & $1.07 \pm 0.12$ & $1.04 \pm 0.12$ & 0.00 & 0.00 & $0.00 \pm 0.25$ & $0.00 \pm 1.00$ \\
\hline $3 \mathrm{C} 286$ & $1.10 \pm 0.15$ & $1.10 \pm 0.15$ & $1.10 \pm 0.01$ & 0.09 & 0.00 & $0.08 \pm 0.10$ & $0.00 \pm 0.48$ \\
\hline $4 \mathrm{C} 12.50$ & $2.07 \pm 0.10$ & $2.10 \pm 0.11$ & $2.09 \pm 0.06$ & 0.33 & 0.66 & $0.16 \pm 0.11$ & $0.31 \pm 0.54$ \\
\hline 3C298 & $2.05 \pm 0.11$ & $2.06 \pm 0.12$ & $2.06 \pm 0.23$ & 0.15 & 0.00 & $0.07 \pm 0.08$ & $0.00 \pm 0.38$ \\
\hline UGC09799 & $2.90 \pm 0.14$ & $2.91 \pm 0.14$ & $2.91 \pm 0.03$ & 0.17 & 0.00 & $0.06 \pm 0.49$ & $0.00 \pm 1.00$ \\
\hline $4 C 04.51$ & $4.02 \pm 0.17$ & $4.06 \pm 0.17$ & $3.99 \pm 0.04$ & 1.13 & 0.00 & $0.28 \pm 0.05$ & $0.00 \pm 0.24$ \\
\hline 3C $327.1 \mathrm{~A}$ & $7.21 \pm 0.17$ & $7.87 \pm 0.18$ & $7.87 \pm 0.19$ & 3.34 & 0.00 & $0.42 \pm 0.12$ & $0.00 \pm 0.47$ \\
\hline $3 \mathrm{C} 327.1 \mathrm{~B}$ & $7.21 \pm 0.17$ & $7.83 \pm 0.18$ & $7.87 \pm 0.21$ & 3.26 & 0.00 & $0.41 \pm 0.11$ & $0.00 \pm 0.44$ \\
\hline PKS1607 & $3.78 \pm 0.14$ & $3.88 \pm 0.14$ & $3.86 \pm 0.11$ & 0.89 & 0.00 & $0.23 \pm 0.09$ & $0.00 \pm 0.20$ \\
\hline $\mathrm{J} 1613$ & $1.50 \pm 0.14$ & $1.50 \pm 0.14$ & $1.50 \pm 0.14$ & 0.00 & 0.00 & $0.00 \pm 0.16$ & $0.00 \pm 0.80$ \\
\hline $3 \mathrm{C} 345$ & $0.71 \pm 0.06$ & $0.71 \pm 0.06$ & $0.71 \pm 0.06$ & 0.00 & 0.00 & $0.00 \pm 0.29$ & $0.00 \pm 1.00$ \\
\hline $3 \mathrm{C} 346$ & $5.00 \pm 0.15$ & $5.19 \pm 0.16$ & $5.21 \pm 0.10$ & 1.45 & 0.00 & $0.28 \pm 0.09$ & $0.00 \pm 0.44$ \\
\hline 3C390 & $25.05 \pm 1.13$ & $25.91 \pm 1.17$ & $26.00 \pm 0.80$ & 3.05 & 10.52 & $0.12 \pm 0.02$ & $0.40 \pm 0.08$ \\
\hline 4C 33.48 & $13.48 \pm 0.39$ & $14.04 \pm 0.40$ & $13.80 \pm 0.44$ & 1.58 & 0.00 & $0.11 \pm 0.06$ & $0.00 \pm 0.27$ \\
\hline 3C409A & $23.00 \pm 0.80$ & $27.74 \pm 0.96$ & $27.05 \pm 2.27$ & 12.96 & 0.63 & $0.48 \pm 0.19$ & $0.02 \pm 0.08$ \\
\hline $3 \mathrm{C} 409 \mathrm{~B}$ & $23.00 \pm 0.80$ & $27.66 \pm 0.96$ & $25.63 \pm 1.85$ & 7.11 & 7.67 & $0.28 \pm 0.21$ & $0.30 \pm 0.08$ \\
\hline $3 \mathrm{C} 410 \mathrm{~A}$ & $38.53 \pm 2.81$ & $54.10 \pm 3.94$ & $53.13 \pm 7.24$ & 24.46 & 0.99 & $0.46 \pm 0.20$ & $0.02 \pm 0.14$ \\
\hline $3 \mathrm{C} 410 \mathrm{~B}$ & $38.53 \pm 2.81$ & $53.31 \pm 3.88$ & $42.87 \pm 18.06$ & 16.17 & 0.00 & $0.38 \pm 0.31$ & $0.00 \pm 0.08$ \\
\hline B2050 & $23.59 \pm 1.20$ & $24.56 \pm 1.25$ & $24.54 \pm 0.84$ & 3.49 & 3.79 & $0.14 \pm 0.11$ & $0.15 \pm 0.05$ \\
\hline $3 \mathrm{C} 433$ & $8.38 \pm 0.04$ & $8.88 \pm 0.04$ & $8.86 \pm 0.29$ & 2.11 & 0.00 & $0.24 \pm 0.10$ & $0.00 \pm 0.39$ \\
\hline PKS2127 & $4.62 \pm 0.28$ & $4.71 \pm 0.29$ & $4.70 \pm 0.24$ & 0.67 & 1.59 & $0.14 \pm 0.04$ & $0.34 \pm 0.19$ \\
\hline J2136 & $4.36 \pm 0.05$ & $4.49 \pm 0.06$ & $4.40 \pm 0.20$ & 0.68 & 0.00 & $0.16 \pm 0.07$ & $0.00 \pm 0.34$ \\
\hline $\mathrm{J} 2232$ & $4.88 \pm 0.16$ & $5.00 \pm 0.16$ & $4.98 \pm 0.07$ & 1.48 & 0.00 & $0.30 \pm 0.10$ & $0.00 \pm 0.26$ \\
\hline $3 \mathrm{C} 454.3$ & $6.84 \pm 0.08$ & $7.05 \pm 0.08$ & $6.91 \pm 0.56$ & 1.97 & 0.00 & $0.28 \pm 0.10$ & $0.00 \pm 0.21$ \\
\hline $3 \mathrm{C} 459$ & $5.43 \pm 0.16$ & $5.60 \pm 0.16$ & $5.56 \pm 0.71$ & 0.32 & 4.27 & $0.06 \pm 0.33$ & $0.77 \pm 0.29$ \\
\hline
\end{tabular}

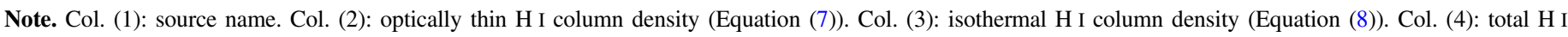

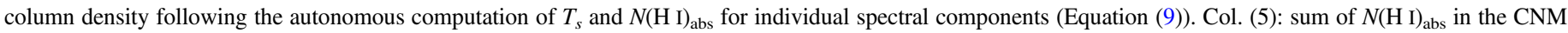

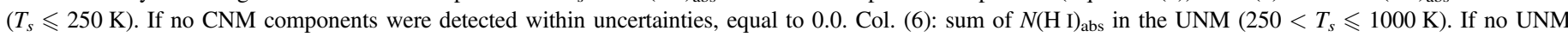

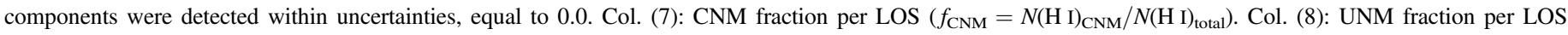
$\left(f_{\mathrm{UNM}}=N(\mathrm{H} \mathrm{I})_{\mathrm{UNM}} / N(\mathrm{H} \mathrm{I})_{\text {total }}\right)$. 

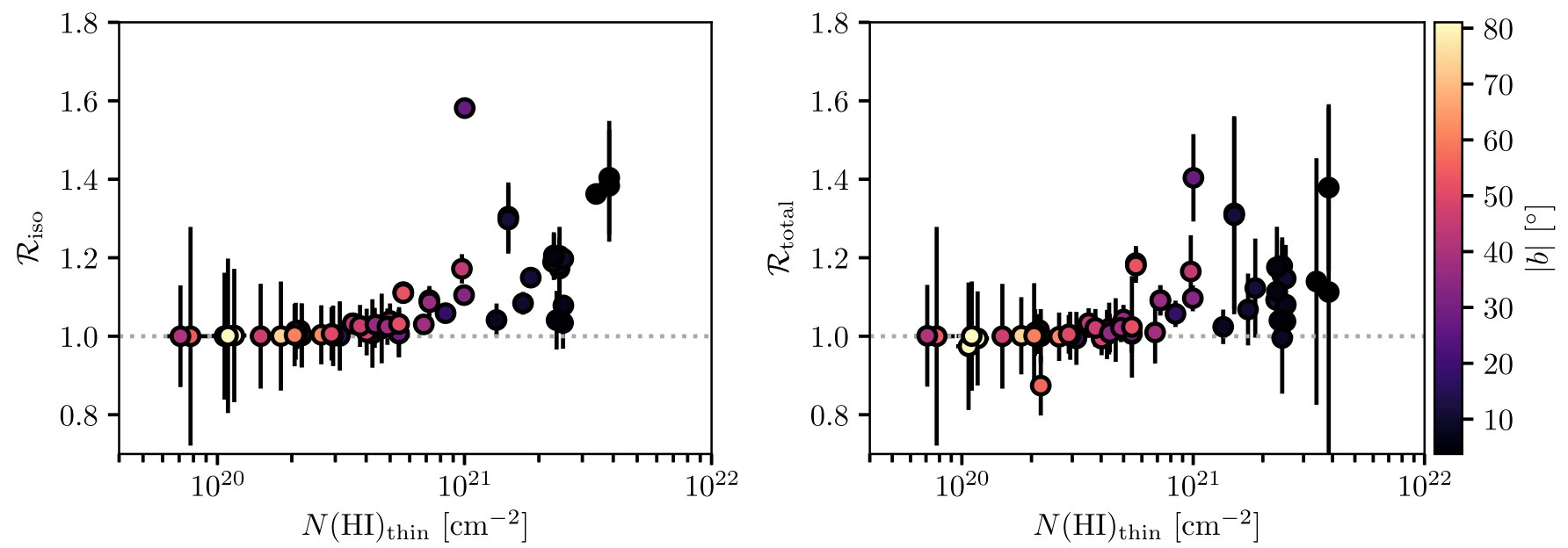

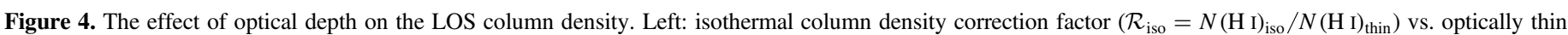

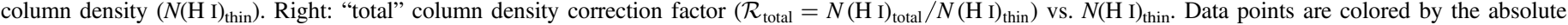
Galactic latitude of the LOS $(|b|)$.

trials used to estimate the uncertainty in $N(\mathrm{H} \mathrm{I})_{\text {total }}$. Next, we compute upper limits to the column density of CNM and UNM below our sensitivity limit by integrating the uncertainty spectrum $\left(\sigma_{\tau}(v)\right)$ and assuming $T_{s}=100 \mathrm{~K}$ and $T_{s}=500 \mathrm{~K}$ for the CNM and UNM, respectively. The final uncertainty is the maximum value between these two estimates.

Keeping in mind the large uncertainties in the LOS mass fractions, we observe most LOSs are roughly less than or roughly half made up of CNM by mass. This is in agreement with a similar analysis of $21 \mathrm{~cm}$ absorption line pairs within and around the Perseus molecular cloud (Stanimirović et al. 2014), and also with HT03. All LOSs feature a significant fraction of WNM by mass, detected only in emission. We will return to discuss the overall mass fractions in the CNM, UNM, and WNM in Section 6.

In Figure 5 we plot $f_{\mathrm{CNM}}$, versus the optical-depth-weighted harmonic mean spin temperature, $\left\langle T_{s}\right\rangle$, given by

$$
\left\langle T_{s}\right\rangle=\frac{\int \tau(v) \cdot T_{s}(v) d v}{\int \tau(v) d v}=\frac{\int \tau(v) \cdot \frac{T_{B}(v)}{(1-\exp (-\tau(v))} d v}{\int \tau(v) d v} .
$$

Although the uncertainty in $f_{\mathrm{CNM}}$ is large, the observed data points cluster around a relation given by $f_{\mathrm{CNM}}=T_{s} /\left\langle T_{s}\right\rangle$ for $T_{s}=77 \pm 57 \mathrm{~K}$ (corresponding to the mean and standard deviation of $T_{s}$ for the CNM). This behavior is consistent with previous observational results (Stanimirović et al. 2014; Murray et al. 2015), as well as with KOK14, and suggests that $\left\langle T_{s}\right\rangle$ may be used as an alternative tracer for $f_{\mathrm{CNM}}$. Future observations at high latitude, where minimal line blending reduces the uncertainty in parameter decomposition, will be important for testing this hypothesis further.

In Figure 6, we plot the all-sky distributions of $f_{\mathrm{CNM}}$ (top), $f_{\text {UNM }}$ (middle), and $\left\langle T_{s}\right\rangle$ (bottom). The highest Galactic latitudes are dominated by WNM (generally low $f_{\mathrm{CNM}}$ and $f_{\mathrm{UNM}}$ ), whereas low Galactic latitudes feature the largest $f_{\mathrm{CNM}}$. Sight lines with the largest $\left\langle T_{s}\right\rangle$, as shown in Figure 5, feature small $f_{\mathrm{CNM}}$ and intermediate $f_{\mathrm{UNM}}$.

We explore the effect of interstellar environment further in Figure 7, where we compare $\left\langle T_{s}\right\rangle$ with dust temperature $T_{d, \text { Planck }}$, derived from modified blackbody fits to Planck observations at 353,545 , and $857 \mathrm{GHz}$, as well as to IRAS

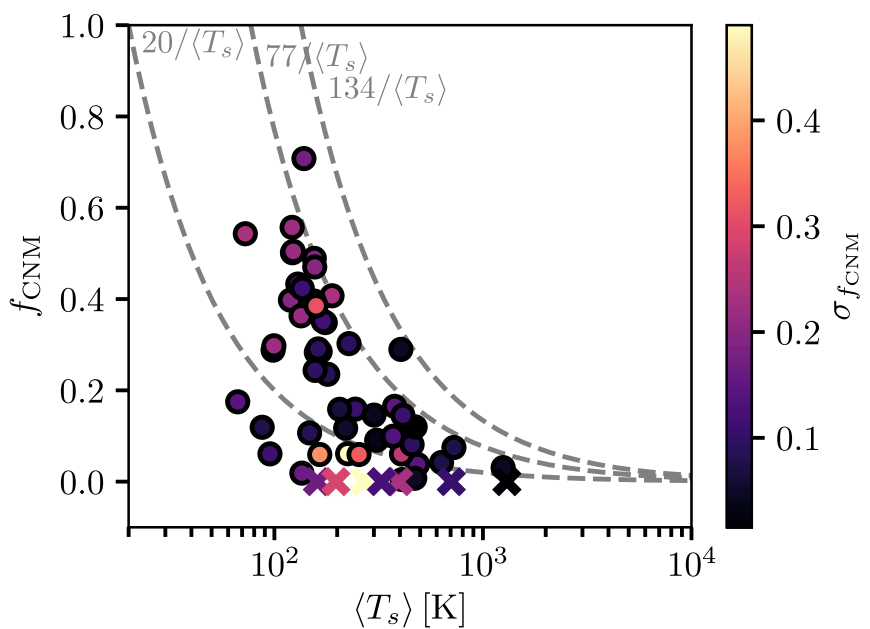

Figure 5. Optical-depth-weighted average spin temperature along the LOS $\left(\left\langle T_{s}\right\rangle\right)$ vs. CNM fraction $\left(f_{\mathrm{CNM}}\right)$. Dashed lines denote $T_{s} /\left\langle T_{s}\right\rangle$ for $T_{s}=77 \pm$ $57 \mathrm{~K}$ (the mean \pm the standard deviation $T_{s}$ for the CNM). Crosses indicate LOSs with $f_{\mathrm{CNM}}=0$, and points are colored by the uncertainty in $f_{\mathrm{CNM}}$ $\left(\sigma_{f_{\mathrm{CNM}}}\right)$. Despite the large scatter, as the majority of observed data points are consistent with the dashed lines, this suggests that $\left\langle T_{s}\right\rangle$ may be used as a tracer of $f_{\mathrm{CNM}}$.

$100 \mu \mathrm{m}$ emission (Planck Collaboration et al. 2014). The dust temperature is a first-order approximation of the interstellar radiation field, which will affect the neutral gas temperature distribution. There is considerable scatter within a narrow range of observed $T_{d \text {,Planck }}$; however, there is a very weak discernible linear trend $(p=0.005)$ of increasing $\left\langle T_{s}\right\rangle$ with increasing $T_{d \text {,Planck }}$, illustrated by the mean and standard deviation $T_{d \text {,Planck }}$ in bins of increasing $\left\langle T_{s}\right\rangle$ (red crosses). In agreement, Kalberla $\&$ Haud (2018) observed that $T_{d \text {,Planck }}$ decreases in regions dominated by the CNM, which correspond to low $\left\langle T_{s}\right\rangle$. Taken together with the trends observed in Figure 6, this provides tentative evidence that stronger interstellar radiation fields result in smaller CNM fractions.

\subsection{Mass Distribution of $H \mathrm{I}$ as a Function of $\mathrm{T}_{\mathrm{s}}$}

In Figure 8, we display a histogram (top panel) and CDF (bottom panel) of the fraction of the total column density detected in absorption and emission over all LOSs per $T_{s}$ bin 

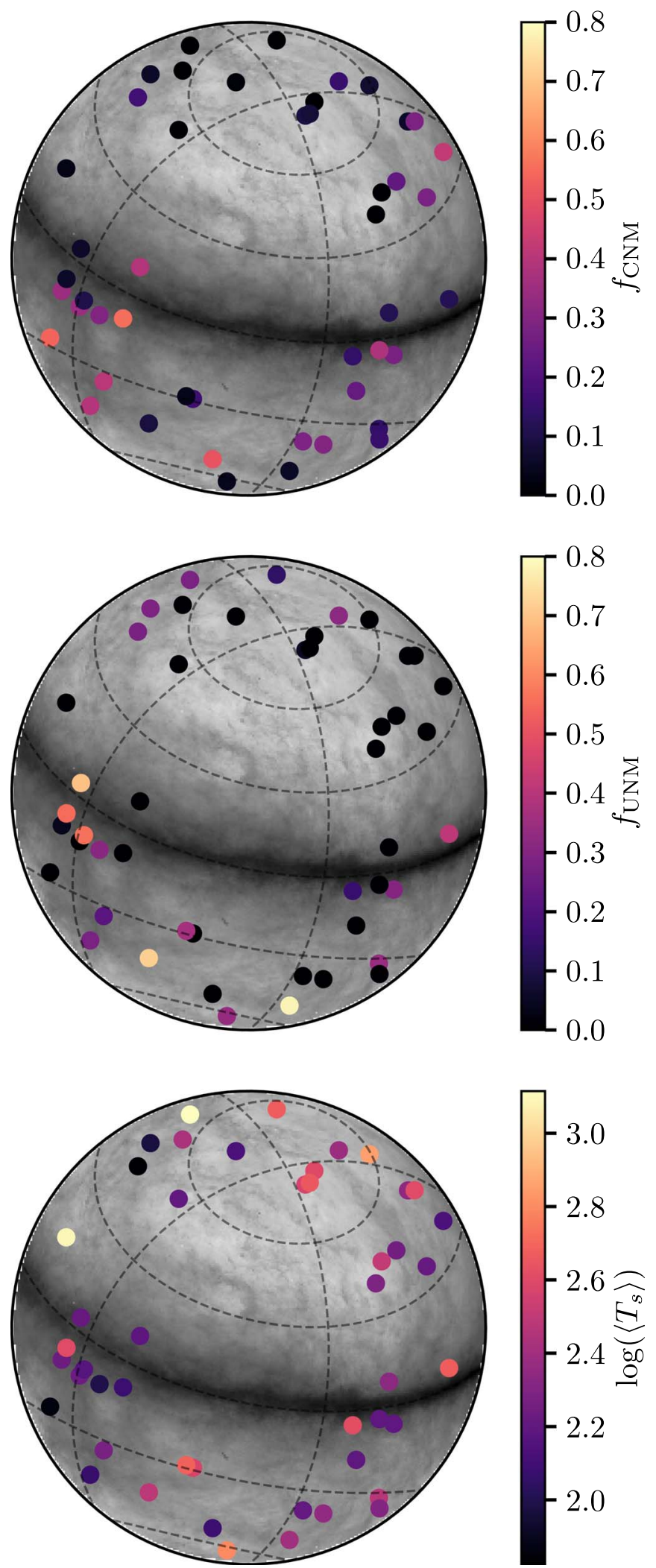

Figure 6. All-sky plots of CNM fraction $\left(f_{\mathrm{CNM}}\right.$; left), UNM fraction $\left(f_{\mathrm{UNM}}\right.$; right), and optical depth-weighted harmonic mean spin temperature $\left(\left\langle T_{s}\right\rangle\right.$; right), overlaid on $\mathrm{H}$ I column density maps in ZEA projection from EBHIS (Winkel et al. 2016).

( $\left.d N(\mathrm{H} \mathrm{I}) / d T_{s}\right)$, a quantity that we denote the "gas fraction" as a function of spin temperature. In the bottom panel, we include CDFs of the results of M15 (purple dashed) for comparison.

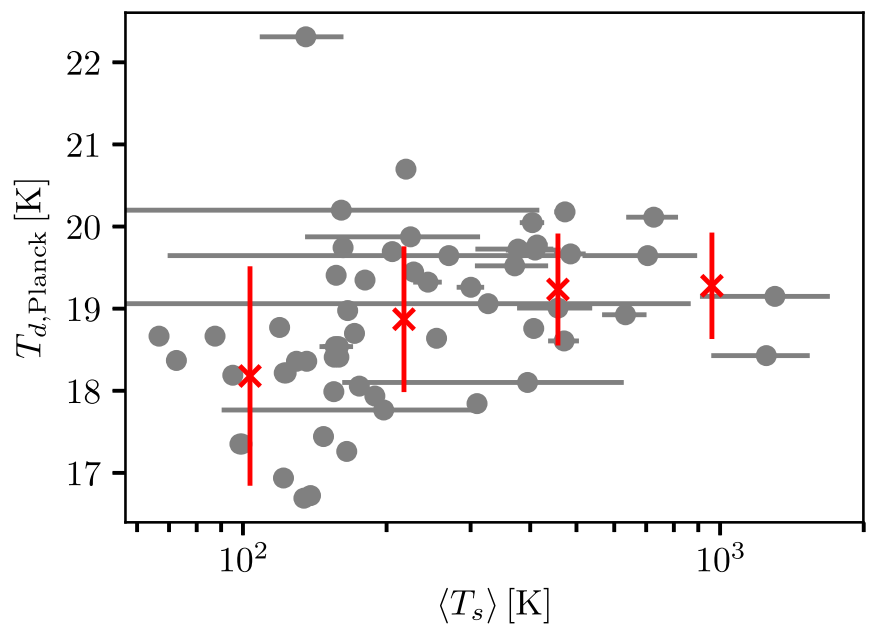

Figure 7. Optical-depth-weighted average spin temperature $\left(\left\langle T_{s}\right\rangle\right)$ vs. dust temperature $\left(T_{d \text {,Planck }}\right)$. The mean and standard deviation over $T_{d \text {,Planck }}$ in bins of increasing $\left\langle T_{s}\right\rangle$ are plotted as red crosses.

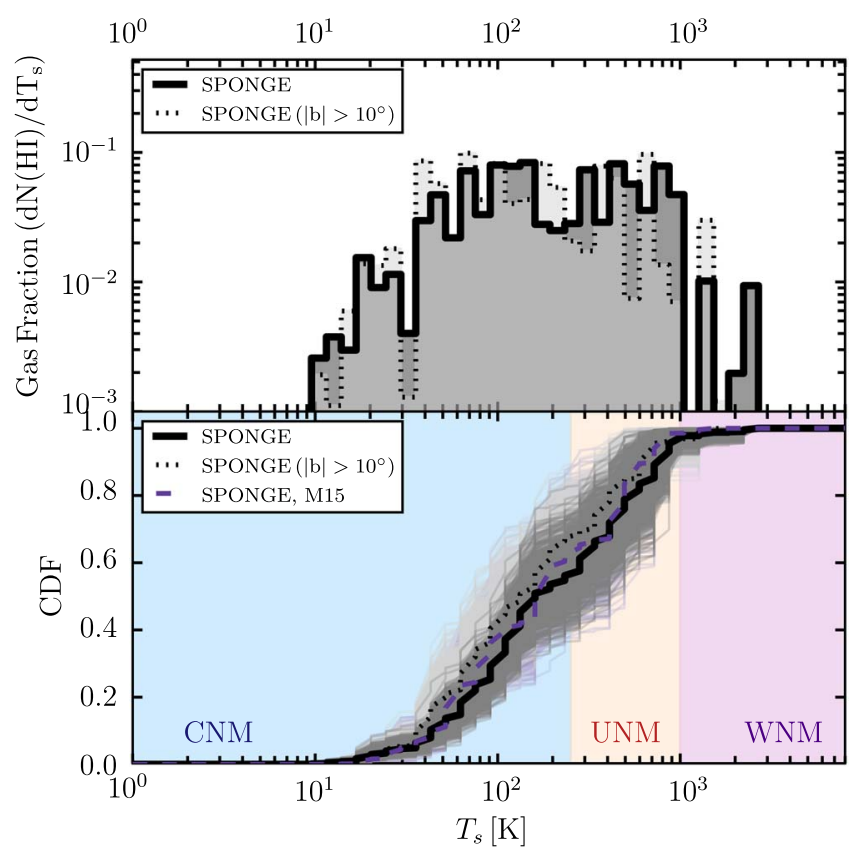

Figure 8. Fraction of total column density detected by 21-SPONGE in absorption as a function of spin temperature for the full sample of 222 absorption components (solid) and the 134 components detected in highlatitude $\left(|b|>10^{\circ}\right)$ LOS (dotted). The results from the first half of the 21-SPONGE survey are shown for comparison (purple dashed; M15). Top: histogram. Bottom: cumulative distribution function (CDF). Approximate spin temperature ranges corresponding to the CNM $\left(T_{s} \leqslant 250 \mathrm{~K}\right)$, UNM $\left(250<T_{s}<1000 \mathrm{~K}\right)$, and WNM $\left(T_{s}>1000 \mathrm{~K}\right)$ are shaded in blue, orange, and purple, respectively.

We bootstrap the observed distributions over 1000 trials and include each resampled CDF to illustrate the effect of outliers on the distribution shapes.

To test the effect of interstellar environment on the observed gas fractions, we isolate the 134 components found at high latitudes $\left(|b|>10^{\circ}\right)$ and include the results for this subsample (black dotted). Sight lines at low Galactic latitude feature increased spectral line complexity due to blending of overlapping structures in radial velocity and also probe different Galactic conditions. We find no significant difference in the observed gas fraction as a function of spin temperature between 
Table 3

H I Mass Fractions

\begin{tabular}{lcc}
\hline \hline Phase & Absorption & Total \\
\hline CNM & $0.56 \pm 0.10$ & 0.28 \\
UNM & $0.41 \pm 0.10$ & 0.20 \\
WNM & $0.03 \pm 0.05$ & 0.52 \\
\hline
\end{tabular}

Note. To compute the "Absorption" mass fractions, we adopt definitions of each phase as follows: over all bootstrapped trials displayed in Figure 8, the maximum $T_{s}$ varies between 150 and $350 \mathrm{~K}$ (Wolfire et al. 2003, cf. their Table 3), the minimum WNM $T_{s}$ varies between 1000 and $4000 \mathrm{~K}$ (Liszt 2001), and the UNM occupies the intervening $T_{s}$. The "Total" mass fractions are computed by incorporating the $50 \%$ of the total $\mathrm{H} \mathrm{I}$ mass detected in emission only, which we assume is WNM.

the full and high-latitude samples. We will discuss this further in Section 6.2.

In Table 3 we list the total mass fractions of gas detected in absorption from each ISM phase in 21-SPONGE. These fractions and their uncertainties were computed as the mean and standard deviation over bootstrapped trials (Figure 8). In each trial, using the range of predicted $T_{s}$ from Wolfire et al. (2003, cf. their Table 3), we allowed the maximum spin temperature definition of the CNM to vary from $T_{s}<150-350 \mathrm{~K}$ and the definition of the WNM to vary from $T_{s}>1000-4000 \mathrm{~K}$ (with the UNM defined as the intervening temperatures) to incorporate the uncertainty in these definitions. We also include the fractions of the total H I column density in each phase, with the assumption that the $50 \%$ of the total $\mathrm{H}$ I column density detected by $21-\mathrm{SPONGE}$ in emission alone is from the WNM.

\subsection{Estimating Observational Bias}

To address the bias imposed by our analysis method on the observed 21-SPONGE mass distribution (i.e., bias toward certain spectral line shapes imposed by AGD, or observational sensitivity limits), we compare HI mass distributions from simulated data. First, in an identical manner to the 21-SPONGE distributions shown in Figure 8, we compute the fraction of total column density in each $T_{s}$ bin from the Gaussian decomposition of the 9355 synthetic spectral line pairs from KOK14 with maximum WF. For each LOS, we then extract the density $(n)$ and temperature $(T)$ as a function of distance $(s)$ from the KOK13 simulation used to construct the synthetic spectra in KOK14. To compare with the "observed" mass distribution of $\mathrm{HI}$ as a function of $T_{s}$, we estimate the "true" underlying mass distribution by computing the fraction of total $n$ per $T_{s}$ bin for all gas along the KOK13 simulated LOS from which the KOK14 spectra were computed. We display both distributions in the top panel of Figure 9.

To emphasize the influence of the WF prescription on these results, in the top panel of Figure 9, we also include the mass distribution as a function of $T_{s}$ from the KOK13 simulation under the assumption of a constant Ly $\alpha$ radiation field density $\left(10^{-6} \mathrm{~cm}^{-3}\right.$; dashed blue) for comparison. Although the mass fractions in the CNM are unchanged between the "constant" and "maximum" WF effect implementation, the peak of the WNM spin temperature distribution changes from $\sim 4000$ to $\sim 6000 \mathrm{~K}$. Clearly, the implementation of the WF effect has the potential to change the WNM spin temperature distribution dramatically.

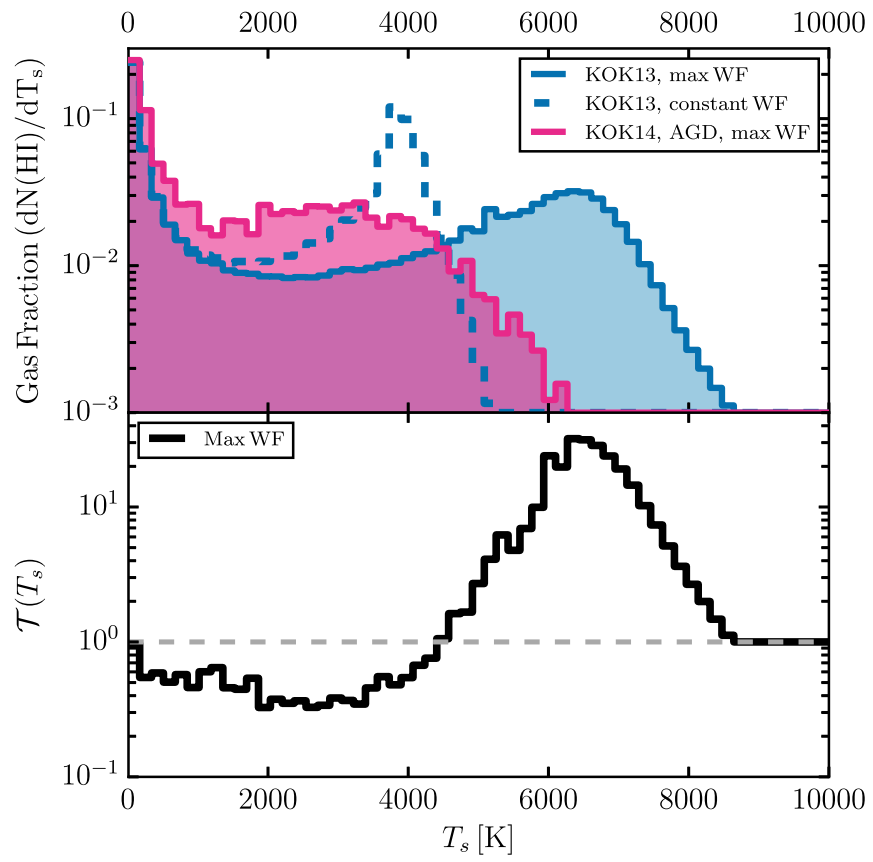

Figure 9. Top: fraction of total column density as a function of spin temperature computed from the KOK13 simulation with maximum WF (i.e., $T_{s}=T_{k}$; solid blue), with constant WF (dashed blue), and from the AGD analysis of KOK14 (pink). Bottom: transfer function, $\mathcal{T}\left(T_{s}\right)$, derived by comparing the temperatures and densities of ISM simulated by KOK13 with those inferred from the synthetic observations of KOK14 (Equation (12)).

By comparing the KOK13 and KOK14 distributions in Figure 9 (with max WF) in detail, we can quantify the differences between observed and simulated $\mathrm{H}$ I mass distributions. Specifically, we compute a "transfer function," $\mathcal{T}\left(T_{s}\right)$, where

$$
\left(\frac{d N(\mathrm{H} \mathrm{I})}{d T_{s}}\right)_{\text {true }}=\mathcal{T}\left(T_{s}\right) \cdot\left(\frac{d N(\mathrm{H} \mathrm{I})}{d T_{s}}\right)_{\text {obs }} .
$$

Assuming that KOK13 and KOK14 trace the "true" and observed gas fractions respectively, we have

$$
\mathcal{T}\left(T_{s}\right)=\left(\frac{d N(\mathrm{H} \mathrm{I})}{d T_{s}}\right)_{\mathrm{KOK} 13} /\left(\frac{d N(\mathrm{H} \mathrm{I})}{d T_{s}}\right)_{\mathrm{KOK} 14, \mathrm{AGD}} .
$$

We display $\mathcal{T}\left(T_{s}\right)$ for all bins with $d N(\mathrm{H} \mathrm{I}) / d T_{s}>0.001$ in the bottom panel of Figure 9. The shape of the transfer function encodes biases in our AGD-based analysis method's recovery of a realistic H I mass distribution. In addition, $\mathcal{T}\left(T_{s}\right)$ encodes the radiative transfer and WF prescription required to go from $\left(n, T_{k}\right)$ in KOK13 to synthetic spectra in KOK14, as well as observational sensitivity limitations imposed by adding synthetic noise to the KOK14 spectra. We emphasize that $\mathcal{T}\left(T_{s}\right)$ will be used in this work to qualitatively assess these limitations. In the future, a full library of simulations and synthetic observations is required to find the best fit with observations and to correct observed gas fractions quantitatively.

\section{Discussion}

With the preceding analysis in hand, we will discuss the observed properties of Galactic H I from 21-SPONGE, aided by the concurrent analysis of synthetic $21 \mathrm{~cm}$ spectral lines from numerical simulations. As numerical simulations produce models of the ISM with increasing resolution, precision, and 


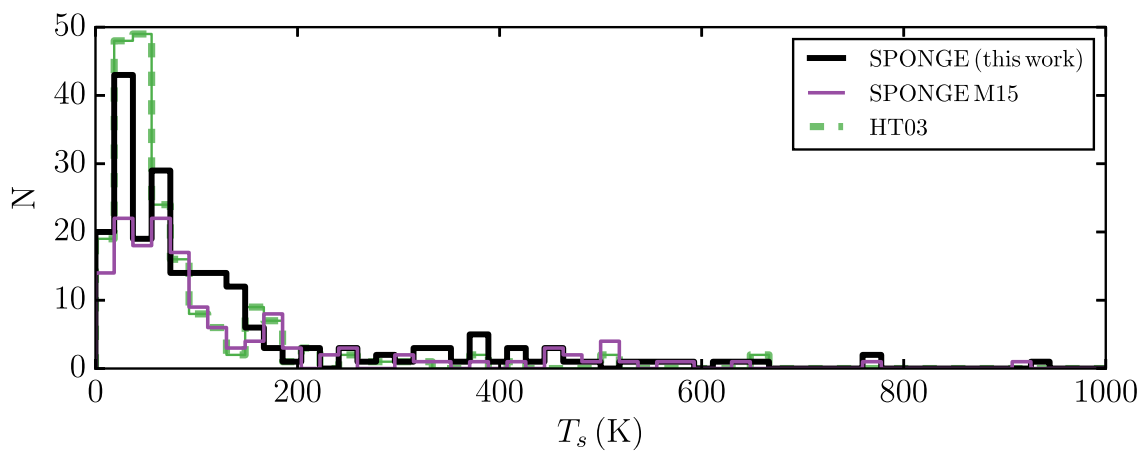

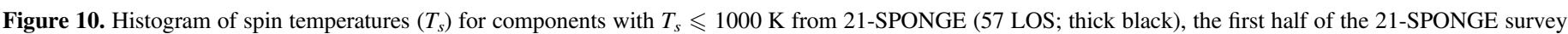

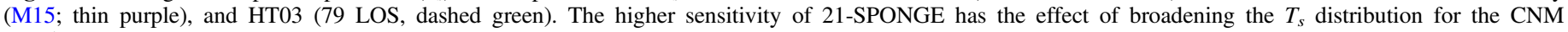
$\left(T_{s} \lesssim 200 \mathrm{~K}\right)$.

accuracy, and as wide field surveys at next-generation observatories roll in, this is likely to become an increasingly common practice. We emphasize that the AGD-based decomposition and radiative transfer approach presented here was designed to be applied to real and synthetic data volumes which are too large to analyze by hand, so that this study may serve as a pilot for future surveys.

So, what have we learned from 21-SPONGE? In this section, we discuss salient properties of the three canonical H I phases, the CNM, UNM, and WNM.

\subsection{The CNM is Ubiquitous}

Of all the HI phases, we are most successful in recovering the physical properties of the CNM. From a detailed comparison between individual clouds along simulated KOK13 LOSs and decomposed spectral features from KOK14 synthetic spectra, we demonstrated that our spectral analysis method not only recovers the majority of "real" CNM clouds, but successfully infers their column densities and spin temperatures (Murray et al. 2017). Furthermore, we observe that the transfer function in Figure 9 is $\sim 1$ in the CNM regime at low $T_{s}$, indicating that our the AGD-plus-radiative transfer method is relatively complete in its recovery of the overall fraction of $\mathrm{H}$ I mass in the CNM.

21-SPONGE has established that with improved sensitivity in optical depth, signatures of the CNM in the form of weak, narrow absorption lines are detected ubiquitously. This agrees with Stanimirović \& Heiles (2005), who found that increased integration time on several nondetection sight lines from HT03 revealed CNM absorption features. Out of 57 total LOSs, we have only 7 nondetections of the CNM $(\sim 88 \%$ detection rate). For five of these sources (3C 236, 3C 245B, 4C 25.43, J1613, 3C 345), no absorption was detected above $3 \sigma_{\tau}(v)$, and for two sources (3C 245A, 1055+018), no detected components featured $T_{s} \leqslant 250 \mathrm{~K}$ within uncertainties from the AGD-based fit. Furthermore, we detect the majority of $\mathrm{HI}$ mass in absorption from the CNM, $56 \% \pm 10 \%(28 \%$ of the total H I mass including WNM detected only in emission).

However, despite the ubiquity of the CNM, the integrated optical depths are low enough that the correction to the total column density for the presence of cold, optically thick H I is relatively small. As shown in Figure 4, it is only sources at low Galactic latitudes $\left(|b| \lesssim 10^{\circ}\right)$ that exhibit correction factors significantly greater than unity. Our results agree with previous studies of $21 \mathrm{~cm}$ absorption to infer the optical depth correction to the H I column density budget (Heiles \& Troland 2003a; Lee et al. 2015; Reach et al. 2017; Nguyen et al. 2018). For example, Lee et al. (2015) analyzed 26 sight lines within and around the Perseus molecular cloud to estimate the contribution of optically thick H I to the "dark gas" budget (i.e., gas undetected in $\mathrm{H}$ I or $\mathrm{CO}$ emission). Applying a trend similar to that observed in Figure 4, they found that the total H I mass of Perseus increases by only $10 \%$ due to optically thick $\mathrm{HI}$. Together, our results contrast recent studies inferring significant corrections ( $\gtrsim 200 \%)$ to the total gas column density from optically thick $\mathrm{H}$ I based on observed far-infrared properties of dust (e.g., Fukui et al. 2015) and indicate that optically thick $\mathrm{H} \mathrm{I}$ is not likely a dominant component of CO-dark gas in the local ISM (Murray et al. 2018).

To compare with previous studies of CNM properties, in Figure 10 we display histograms of $T_{s}$ below $1000 \mathrm{~K}$ from 21-SPONGE (thick black), M15 (thin purple), and HT03 (dashed green). The HT03 distribution is strongly peaked at $\sim 50 \mathrm{~K}$, indicative of a characteristic $T_{s}$ for the CNM. Although we observe a similar feature in 21-SPONGE, we also find evidence for a broader CNM $T_{s}$ distribution. To compare the statistics in detail, in Table 4 we compute mean and median values for the CNM $T_{s}$ from 21-SPONGE and HT03 (i.e., all components with $T_{s} \leqslant 250 \mathrm{~K}$ ) with and without weighting by $N(\mathrm{HI})_{\mathrm{abs}}$ for two latitude bins, above and below $|b|=10^{\circ}$, following Table 2 of Heiles \& Troland (2003b). Considering the scatter (standard deviations $\gtrsim 50 \mathrm{~K}$ ), the values are generally consistent. We appear to detect higher mean and median CNM $T_{s}$ weighted by $N(\mathrm{HI})_{\text {abs }}$ in 21-SPONGE than HT03, consistent with the observed broadening of the $T_{s}$ histogram in Figure 10.

Ultimately, improving the statistics of the CNM $T_{s}$ distribution will be crucial for understanding the sources of observed differences and scatter between distributions, as environmental effects are likely important. For example, photoelectric heating by dust grains may be enhanced in particularly dust-rich Galactic environments, or strong variations in turbulence may broaden the distribution. Upcoming $21 \mathrm{~cm}$ absorption surveys at the VLA and Australian Square Kilometer Array Pathfinder (GASKAP; Dickey et al. 2013) will dramatically expand the known sample of Galactic H I absorption properties. Although these studies will likely not reach the optical depth sensitivity in individual sight lines achieved by 21-SPONGE, they will be crucial for resolving detailed CNM properties as a function of Galactic environment. 
Table 4

$\mathrm{CNM}$ and $\mathrm{UNM} T_{s}$ Statistics

\begin{tabular}{|c|c|c|c|c|c|c|c|c|c|c|c|}
\hline \multirow[b]{2}{*}{ Latitude } & \multirow[b]{2}{*}{ Survey } & \multirow[b]{2}{*}{$N_{\mathrm{CNM}}$} & \multicolumn{2}{|c|}{ By $N_{\mathrm{CNM}}(\mathrm{K})$} & \multicolumn{2}{|c|}{ By $N(\mathrm{H} \mathrm{I})(\mathrm{K})$} & \multirow[b]{2}{*}{$N_{\mathrm{UNM}}$} & \multicolumn{2}{|c|}{ By $N_{\mathrm{UNM}}(\mathrm{K})$} & \multicolumn{2}{|c|}{ By $N(\mathrm{H} \mathrm{I})(\mathrm{K})$} \\
\hline & & & Mean & Median & Mean & Median & & Mean & Median & Mean & Median \\
\hline High & 21-SPONGE & 109 & 73 & 61 & 95 & 79 & 23 & 450 & 390 & 500 & 460 \\
\hline$\left(|b|>10^{\circ}\right)$ & HT03 & 135 & 63 & 45 & 74 & 56 & 11 & 420 & 380 & 400 & 380 \\
\hline Low & 21-SPONGE & 71 & 68 & 49 & 108 & 106 & 14 & 460 & 430 & 510 & 490 \\
\hline$\left(|b|<10^{\circ}\right)$ & HT03 & 51 & 67 & 47 & 86 & 60 & 2 & 300 & 300 & 260 & 260 \\
\hline
\end{tabular}

Note. Means and medians "by $N$ " are computed for all $N_{\mathrm{CNM}} \mathrm{CNM}$ components $\left(T_{s} \leqslant 250 \mathrm{~K}\right)$ and all $N_{\mathrm{UNM}} \mathrm{UNM}$ components $\left(250 \leqslant T_{s} \leqslant 1000 \mathrm{~K}\right)$ with no weighting; following Heiles \& Troland (2003b), the median "by $N(\mathrm{H} \mathrm{I})$ " is the $T_{s}$ for which half the total CNM or UNM column density lies above and half below, and the mean is weighted by $N(\mathrm{H} \mathrm{I})$.

\subsection{Thermally Unstable Gas Fraction}

A key motivation for the 21-SPONGE survey was to determine the effect of improved optical depth sensitivity on the inferred fraction of $\mathrm{H}$ I mass in the thermally unstable regime (e.g., $250 \lesssim T_{s} \lesssim 1000 \mathrm{~K}$ ). There is substantial evidence in the literature for a significant population of thermally unstable gas in the ISM; however, the majority of constraints on unstable spin temperatures are inferred as upper limits from line-width-based kinetic temperatures (e.g., Verschuur \& Magnani 1994; Heiles \& Troland 2003a; Haud \& Kalberla 2007; Kalberla \& Haud 2018). With detections in $\tau$ and $T_{B}$, we can constrain $T_{s}$ and more accurately assess the thermodynamic state of the neutral ISM.

However, as spectral features corresponding to warmer gas are characterized by broader line widths, spectral complexity due to velocity blending increases the difficulty in recovering accurate gas density and temperature from both $21 \mathrm{~cm}$ absorption and emission. We quantified this effect in Murray et al. (2017) by showing that our decomposition and radiative transfer approach tends to overestimate the temperatures of non-CNM structures $\left(T_{s} \gtrsim 400 \mathrm{~K}\right)$. In that study, we were primarily sensitive to gas with $T_{s}<1000 \mathrm{~K}$ (i.e., CNM and UNM) for which we could unambiguously identify "true" simulated counterpart structures along the simulated LOS. Considering this bias, the mass fraction of thermally unstable $\mathrm{HI}$ in absorption presented here is possibly an upper limit, as CNM with overestimated $T_{s}$ may contribute. We also note that in the expected range of thermally unstable temperatures $\left(250 \lesssim T_{s} \lesssim 1000 \mathrm{~K}\right)$, we observe that $\mathcal{T}\left(T_{s}\right)$ is relatively flat but $<1$ (Figure 9), indicating that our analysis method is not only sensitive to these conditions but that we tend to overestimate the true mass fraction.

To illustrate the typical UNM properties detected by 21-SPONGE (in comparison with HT03), in Table 4 we display mean and median values for the UNM $T_{s}$ from 21-SPONGE and HT03 (i.e., all absorption components with $250<T_{s}<1000 \mathrm{~K}$ ) with and without weighting by $N(\mathrm{H} \mathrm{I})_{\text {abs }}$ for high and low Galactic latitudes. The statistics are much poorer for comparing UNM properties (i.e., HT03 only find two UNM components at low latitudes, due to limited observational sensitivity); however, the observed UNM properties appear to be largely consistent with each other at both high and low latitudes.

Our estimate of the thermally unstable gas fraction $(41 \%$ $\pm 10 \%$ of $\mathrm{HI}$ detected in absorption by mass, and $\sim 20 \%$ of the total observed HI mass) is generally consistent with previous observational results. Although Kalberla \& Haud (2018) find a larger UNM fraction- $41 \%$ of the local H I mass-they acknowledge that the systematic uncertainties on this estimate are likely very large, up to $50 \%$. As discussed above, identifying spectral features from the UNM is complicated by uncertainties in the definition of CNM and WNM thresholds, and the fact that the UNM is typically strongly blended with CNM and WNM features. Furthermore, to distinguish between disparate H I phases using $21 \mathrm{~cm}$ emission and $T_{k, \max }$ alone requires uncertain assumptions regarding the contributions of thermal and nonthermal broadening to the observed line widths. Without sufficient observational sensitivity to detect UNM consistently in $21 \mathrm{~cm}$ absorption, HT03 determined that $\sim 48 \%$ of the total WNM column density beyond the Galactic plane, or $\sim 30 \%$ of the total out-of-plane column density, has thermally unstable temperatures. For comparison with our UNM fraction, of the gas detected in absorption by HT03, only $9 \%$ by mass has $250 \leqslant T_{s}<1000 \mathrm{~K}$. With improved $21 \mathrm{~cm}$ absorption sensitivity, a study by Roy et al. (2013b) similar to 21SPONGE argued that at least $28 \%$ of $\mathrm{HI}$ is thermally unstable. Finally, KOK13 also find a substantial fraction of gas out of thermal equilibrium, $\sim 18 \%$ by mass, due to strong turbulence, expanding shocks from supernovae, and a time-dependent heating rate. Below $T_{s} \sim 1000 \mathrm{~K}$, our observed distribution qualitatively agrees with their results (cf. Figure 8(d) of KOK13).

In contrast to HT03, we do not observe a significant difference between the mass distribution of $\mathrm{H} \mathrm{I}$ (including the thermally unstable gas fraction) between the full sample and subsample of sight lines at high Galactic latitude $\left(|b|>10^{\circ}\right)$. Considering that velocity blending and overall fitting uncertainties are higher at lower latitudes, we might expect some kind of bias toward certain gas populations in different regimes. To resolve variations with Galactic environments, we need larger samples of high-sensitivity absorption lines to improve statistics, which may distinguish between regimes where thermal instability, turbulence, and dynamical processes dominate the regulation of the ISM (Wolfire 2015).

\subsection{How "Warm" is the WNM?}

From standard, steady-state ISM models, we expect the WNM to have kinetic temperatures of $T_{k}=5000-10000 \mathrm{~K}$ and spin temperatures of $T_{s} \sim 1000-4000 \mathrm{~K}$ (Liszt 2001; Wolfire et al. 2003). In the WNM, it is typically assumed that $T_{s}<T_{k}$ because collisions are insufficient at low densities for thermalizing the $21 \mathrm{~cm}$ transition. Our AGD analysis of KOK14 spectra demonstrates that we should easily detect WNM in the expected range of spin temperature, despite the limitations of our observational sensitivity. The prominent peak at $T_{s} \sim 2500 \mathrm{~K}$ in the KOK14 histogram of Figure 8 (pink) illustrates the sensitivity of the AGD method to this range of temperature. However, we detect very little $\mathrm{HI}$ mass in absorption from the WNM $(3 \% \pm 5 \%)$.

Consequently, the fact that we do not detect a significant mass fraction of WNM from 21-SPONGE with $T_{s} \gtrsim 1000-4000 \mathrm{~K}$ 
indicates that the WNM spin temperature may be higher than standard analytical predictions, which are based on H I excitation via particle collisions alone (e.g., Liszt 2001). In agreement, Murray et al. (2014) detected an unexpectedly "warm" WNM population with $T_{s}=7200_{-1200}^{+1800} \mathrm{~K}$, which was attributed to supplemental excitation beyond collisions from resonant scattering of Ly $\alpha$ photons (the WF effect; Wouthuysen 1952; Field 1958). In Murray et al. (2017), we found that a simple WF treatment in KOK14 spectra produces spectral features corresponding to expected WNM properties (i.e., $T_{s} \sim 3000-4000 \mathrm{~K}$ ), but which are not detected by 21-SPONGE, and this comparison is what led us to analyze synthetic spectra from KOK14 with "maximum WF" in this study. Sophisticated theoretical treatment of the WF effect in future simulations is of utmost importance, as myriad environmental conditions, including metallicity and cosmic-ray ionization rate, will affect Ly $\alpha$ pumping of the $21 \mathrm{~cm}$ transition (e.g., Shaw et al. 2017).

Ultimately, considering that we analyze the "maximum" WF case of the KOK14 synthetic spectra $\left(T_{s}=T_{k}\right.$ for all $\left.\mathrm{HI}\right)$, we are likely limited by observational sensitivity to detecting gas at higher spin temperatures (i.e., $T_{s} \gtrsim 4000 \mathrm{~K}$ : prominent in the KOK13 mass distribution but missing from the inferred distribution from KOK14). We added synthetic Gaussian noise with $\mathrm{rms}=1 \times 10^{-3}$ to the synthetic absorption spectra from KOK14 to mimic the 21-SPONGE sensitivity, and AGD is unable to recover lines with $T_{s} \gtrsim 4000 \mathrm{~K}$ at $\mathrm{S} / \mathrm{N} \geqslant 3$ with this level of spectral uncertainty. As a result, the majority of $\mathrm{HI}$ mass not detected in absorption ( $50 \%$ of the total LOS column density) is likely in the form of WNM.

To test for the presence of WNM below our sensitivity limit, we performed a simple stacking experiment. First, we subtracted all components detected in both absorption and emission from $\tau(v)$ and $T_{B, \exp }(v)$ to produce residual absorption and emission spectra, $\tau_{\text {resid }}(v)$ and $T_{B \text {,exp,resid }}(v)$, for all LOSs. The residual spectra contain components with unphysical $T_{S}$ (i.e., not successfully fitted to $T_{B \text {,exp }}(v)$ ) and $\mathrm{H}$ I below our sensitivity limit to detect in absorption (i.e., WNM at high temperature). We mask all velocity channels with components detected in $\tau(v)$ but not $T_{B \text {,exp }}(v)$, as these features are likely CNM or UNM, which we did not recover due primarily to beam mismatch effects. We further mask by eye channels with strong residuals due to the oversubtraction of Gaussian components (typically $\sim 10$ channels for roughly half of the LOS). Next, we compute the average of all unmasked velocity channels whose $T_{B \text {,resid }}$ falls into one of four bins (chosen to select significantly detected residual emission in bins of roughly equal sizes), $3 \sigma_{T_{B}} \leqslant T_{B \text {,exp,resid }}<0.9 \mathrm{~K}, \quad 0.9 \leqslant T_{B \text {,exp,resid }}<$ $2.4 \mathrm{~K}$, and $T_{B \text {,exp,resid }}>2.4 \mathrm{~K}$, weighted by $1 / \sigma_{\tau}$. The number of channels in each bin is 2383, 2604, and 2423, respectively,

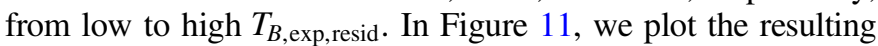
weighted averages of the absorption channels, $\left\langle 1-e^{-\tau_{\text {resid }}(v)}\right\rangle$, and the emission channels $\left\langle T_{B \text {,exp,resid }}\right\rangle$. The weighted average absorption in the bins of smallest $T_{B \text {,exp,resid }}$ is consistent with zero; however, we detect a significant residual absorption signal in the bin of highest $T_{B \text {,exp,resid. The uncertainties are computed }}$ as the standard deviation over $10^{4}$ trials wherein we bootstrapresample the LOS used in the stack with replacement. We denote the constant harmonic mean $T_{s}\left(\left\langle T_{s, \text { resid }}\right\rangle\right)$ with dotted lines and shading in Figure 11, and observe that the significant residual absorption feature is consistent with $\left\langle T_{s \text {,resid }}\right\rangle \sim 10^{4} \mathrm{~K}$.

The inferred spin temperature of the detected residual absorption signal in Figure 11 is consistent with our previous findings

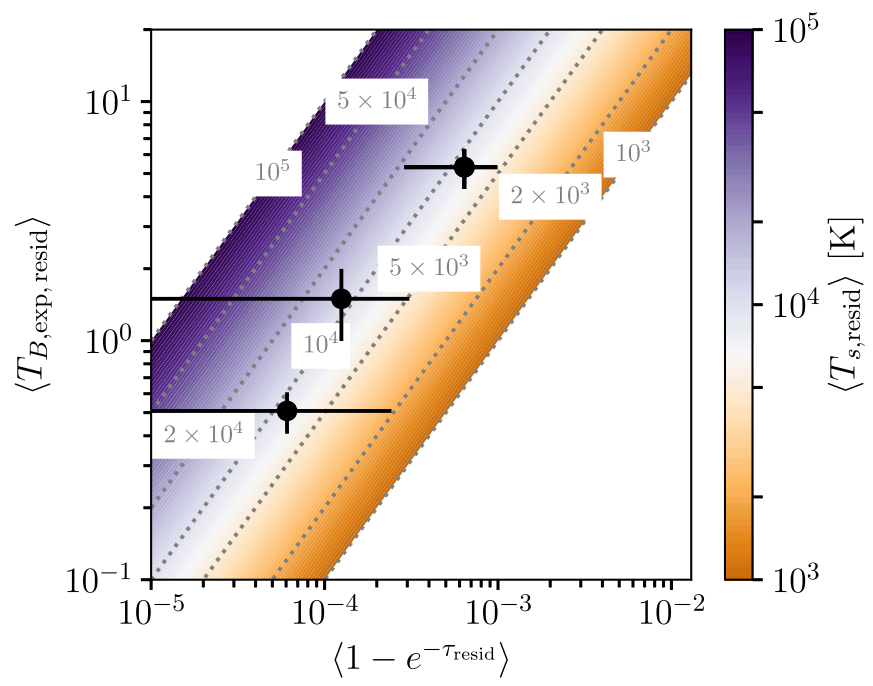

Figure 11. Stacked residual absorption $\left\langle 1-e^{-\tau_{\text {resid }}}\right\rangle$ vs. stacked residual emission $\left\langle T_{B \text {,resid }}\right\rangle$ for all spectral channels binned by residual emission: $3 \sigma_{T_{B}} \leqslant T_{B \text {,resid }}<0.9 \mathrm{~K}, 0.9 \leqslant T_{B \text {,resid }}<2.4 \mathrm{~K}$, and $T_{B \text {,resid }}>2.4 \mathrm{~K}$ (limits chosen to select significantly detected residual emission in three bins of roughly equal sizes). Dotted gray lines, shading, and inset labels denote constant harmonic mean spin temperature for the stacked residual channels $\left(\left\langle T_{s, \text { resid }}\right\rangle\right)$. For the bin of largest residual emission, we detect a significant residual absorption signal, corresponding to $\left\langle T_{s \text {,resid }}\right\rangle \approx 10^{4} \mathrm{~K}$.

$\left(T_{s}=7200_{-1200}^{+1800} \mathrm{~K}\right.$; Murray et al. 2014). Although we do not detect a significant signal in the bins of smallest $T_{B \text {,resid, possibly }}$ due to oversubtraction of Gaussian components in complex LOSs, we note that the mean $\left\langle T_{s, \text { resid }}\right\rangle$ is similar in all bins. This is expected if we are sampling broad spectral line features from a diffuse, warm parent $\mathrm{HI}$ population, rather than randomly sampling occasional peaks in $T_{B}$ or $\tau$ due to fitting imperfections. If the signal detected here (and in Murray et al. 2014) originates from the diffuse WNM with a constant temperature, bins of higher $T_{B \text {,resid }}$ should correspond to higher $\left(1-e^{-\tau_{\text {resid }}}\right)$.

Overall, the detection of a residual absorption signal consistent with high WNM spin temperature further emphasizes the importance of supplementary diffuse neutral gas excitation (e.g., the WF effect) in producing such high $T_{s}$. In addition, future studies aiming to detect the absorbing properties of the WNM will need to target even better optical depth sensitivity than we have achieved with 21-SPONGE or to improve sensitivity by applying stacking techniques similar to those demonstrated in Figure 11 and by Murray et al. (2014).

\section{Summary}

In this work, we present the data release of 21-SPONGE, a large Karl G. Jansky VLA survey for high-sensitivity absorption by Galactic $\mathrm{H}$ I to probe the temperature distribution of the neutral ISM. 21-SPONGE is distinguished among previous $21 \mathrm{~cm}$ studies of the Galactic H I as a result of (1) exceptional optical depth sensitivity $\left(\sigma_{\tau}<10^{-3}\right.$ per $0.42 \mathrm{~km} \mathrm{~s}^{-1}$ channel) thanks to careful calibration considerations and the upgraded capabilities of the VLA WIDAR correlator for producing wide spectral bandwidths and narrow velocity channels for resolving the CNM, UNM, and WNM simultaneously; (2) matching single-dish $21 \mathrm{~cm}$ emission spectra with the highest possible angular resolution $\left(\sim 4^{\prime}\right)$ from the Arecibo Observatory, minimizing the mismatch with subarcminute interferometric VLA absorption measurements; and (3) detailed comparisons with 3D numerical simulations of the ISM for assessing observational biases. In this work, we have 
presented a novel method for autonomously decomposing $21 \mathrm{~cm}$ absorption and emission spectra and deriving the physical properties (column density, temperature) for individual spectral features via detailed radiative transfer. The efficient, objective nature of the analysis method enables us to compare our results with thousands of synthetic observations from numerical simulations. Our main results are summarized here.

1. We demonstrate that with improved optical depth sensitivity, narrow absorption lines arising from the CNM are detected ubiquitously. The detection rate of $21 \mathrm{~cm}$ absorption from the CNM is $\sim 88 \%$. However, the optical depth of these features is typically small so that correction for cold, optically thick gas to the optically thin limit of $\mathrm{HI}$ column density is typically small $(<20 \%)$. Along a typical individual 21-SPONGE LOS, we find the CNM mass fraction to be $\lesssim 50 \%$.

2. To assess the biases of our observational methods, we apply the same analysis techniques to a sample of 9355 synthetic H I spectral line pairs from Kim et al. (2014), constructed from the 3D hydrodynamical simulation by Kim et al. (2013). We add spectral noise to the synthetic data set to mimic the 21-SPONGE sensitivity limits. By comparing the underlying simulated gas properties with those inferred from the synthetic spectral lines, we construct a "transfer function," $\mathcal{T}\left(T_{s}\right)$, between the true and observed mass distributions of $\mathrm{HI}$ as a function of temperature (i.e., by dividing the two distributions). We find that for $T_{s}<4000 \mathrm{~K}, \mathcal{T}\left(T_{s}\right) \sim 1$, indicating that we are sensitive to $\mathrm{H}$ I properties within this regime. At higher $T_{s}$ (i.e., $\left.\gtrsim 4000 \mathrm{~K}\right), \mathcal{T}\left(T_{s}\right)>1$, indicating that our synthetic spectral line analysis is likely missing a significant fraction of WNM mass present in the KOK13 simulation.

3. We compute the fractions of $\mathrm{HI}$ mass detected in emission and absorption (i.e., for which we have constraints on $T_{s}$ for measuring $N(\mathrm{HI})$, corresponding to $50 \%$ of the total H I mass) in the CNM $(56 \% \pm 10 \%)$, WNM $(3 \pm 5 \%)$, and thermally unstable medium (UNM; $41 \% \pm 10 \%)$. Our UNM mass fraction, among the first observational constraints from direct $\mathrm{HI}$ absorption detections, is generally consistent with previous indirect observational estimates (e.g., Heiles \& Troland 2003b). Incorporating the remaining $50 \%$ of $\mathrm{H}$ I mass detected in emission alone (i.e., for which we do not have constraints on $T_{s}$, which we assume is due to WNM), for all 21SPONGE LOSs, the total mass fractions are 28\%, 20\%, and $52 \%$ for the CNM, UNM, and WNM, respectively.

4. Although the WNM makes up the majority of the total $\mathrm{H}$ I mass (52\%), the lack of WNM absorption detected by 21-SPONGE in the spin temperature range expected from steady-state collisional excitation models $\left(T_{s}=\right.$ 1000-4000 K; Liszt 2001; Wolfire et al. 2003) implies that the WNM spin temperature is higher, likely due to supplemental excitation from the WF effect. This is in agreement with previous analysis of 21-SPONGE and the KOK14 synthetic spectra (Murray et al. 2017), as well as stacking analysis of 21-SPONGE spectra, which revealed a high spin temperature WNM population with $T_{s}=7200_{-1200}^{+1800} \mathrm{~K}$ (Murray et al. 2014). As a test of this hypothesis, following spectral line modeling, we stack residual absorption in bins of residual emission and detect a significant absorption feature with a harmonic mean spin temperature $\sim 10^{4} \mathrm{~K}$.
Overall, larger samples of $21 \mathrm{~cm}$ absorption lines, as well as nextgeneration simulations with sophisticated WF treatment, are required to improve the statistical uncertainties and probe the effect of Galactic environment on these results. We emphasize that the autonomous, efficient nature of the AGD method presented here will enable detailed analysis of future real and synthetic data volumes which will be orders of magnitude larger than 21-SPONGE. However, even in the era of next-generation interferometers (e.g., SKA), future surveys will be unlikely to target the superb optical depth sensitivity reached by 21-SPONGE (e.g., McClure-Griffiths et al. 2015), ensuring that this data set will provide an important benchmark for future work.

This work was supported by the NSF Early Career Development (CAREER) Award AST-1056780. C.E.M. acknowledges support by the National Science Foundation Graduate Research Fellowship and the Wisconsin Space Grant Institution. S.S. thanks the Research Corporation for Science Advancement for their support. The authors would like to thank Bob Lindner and Carlos Vera-Ciro for developing AGD, writing GaussPy, and inspiring the methods presented here. C.E.M. thanks Elijah Bernstein-Cooper, Helga Dénes, Katie Jameson, James Dempsey, and Naomi McClureGriffiths for helpful conversations and collaboration toward developing GaussPy. We also thank Van Hiep Nguyen and Joanne Dawson for valuable discussions that led to improved beam efficiency considerations for our expected brightness temperature spectra. This work makes use of data from the Karl G. Jansky Very Large Array, operated by the National Radio Astronomy Observatory (NRAO). NRAO is a facility of the NSF operated under cooperative agreement by Associated Universities, Inc. EBHIS is based on observations with the $100 \mathrm{~m}$ telescope of the MPIfR (Max-Planck-Institut für Radioastronomie) at Effelsberg. The Parkes Radio Telescope is part of the Australia Telescope, which is funded by the Commonwealth of Australia for operation as a National Facility managed by CSIRO. We acknowledge the use of the Legacy Archive for Microwave Background Data Analysis (LAMBDA), part of the High Energy Astrophysics Science Archive Center (HEASARC). HEASARC/LAMBDA is a service of the Astrophysics Science Division at the NASA Goddard Space Flight Center. This research has made use of the NASA/ IPAC Extragalactic Database (NED), which is operated by the Jet Propulsion Laboratory, California Institute of Technology, under contract with the National Aeronautics and Space Administration. This research has made use of NASA's Astrophysics Data System. This research made use of Astropy, a community-developed core Python package for Astronomy (Astropy Collaboration et al. 2013), NumPy (Van Der Walt et al. 2011), and matplotlib, a Python library for publication quality graphics (Hunter 2007).

\section{Appendix}

In this appendix we include supplementary figures and tables to illustrate the 21-SPONGE data products.

Figure 12 displays $1.4 \mathrm{GHz}$ continuum images of the 21-SPONGE target fields centered on compact sources selected from the NVSS survey (Condon et al. 1998). Many sources exhibit significantly resolved structures.

Figure 13 displays the results of autonomous Gaussian decomposition of the 57 21-SPONGE LOS. The velocity component parameters and estimates for the physical properties of the associated HI structures for each LOS are included in Table 5. 

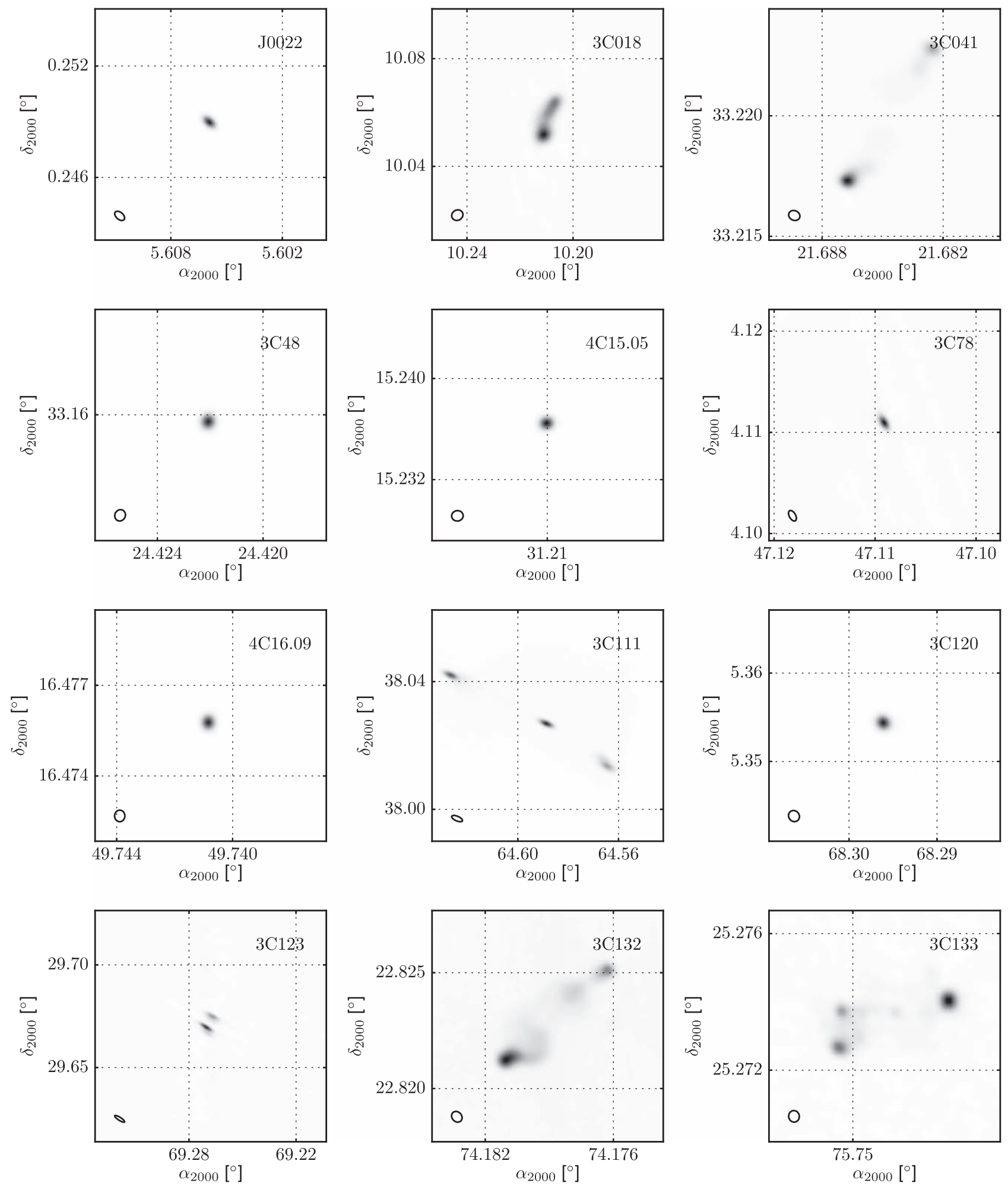

Figure 12. 21-SPONGE $1.42040575 \mathrm{GHz}$ continuum images. The source name is printed within each panel, and the synthesized beam used to construct each image is included in the bottom-left corner. Each image is scaled so that the peak flux density is unity.

(The complete figure set (4 images) is available.) 

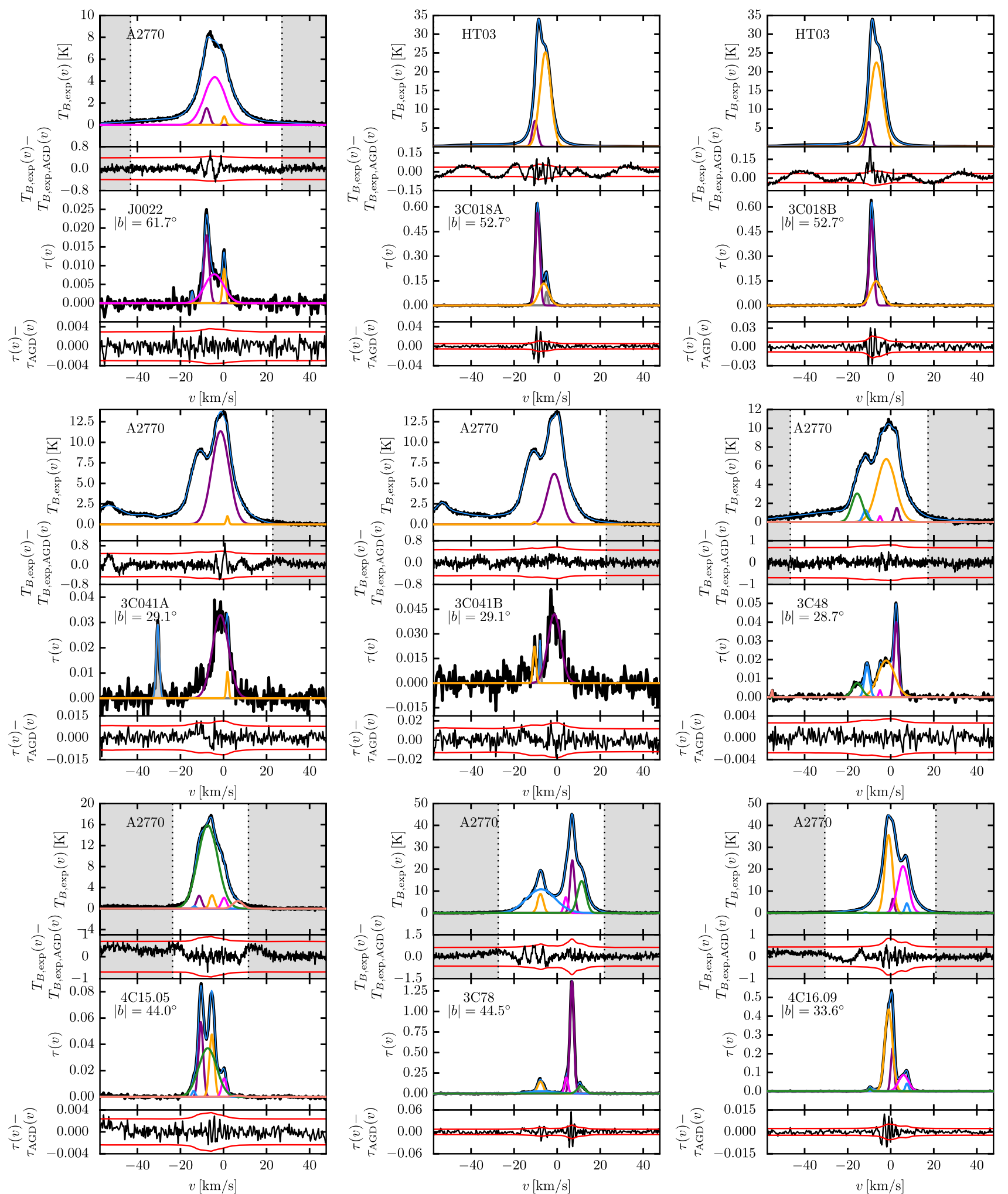

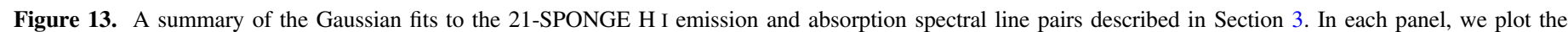

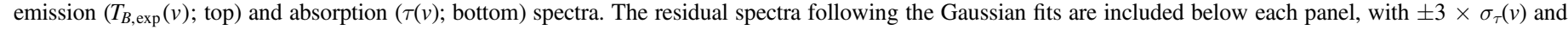

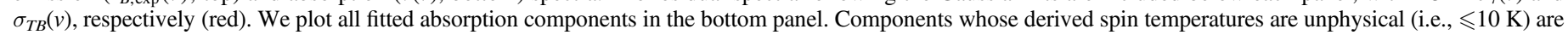

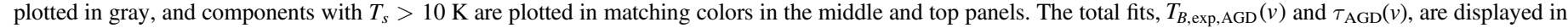

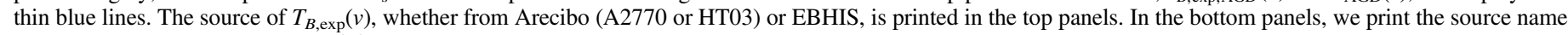

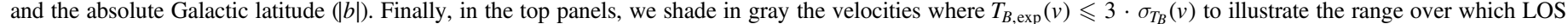
column densities are computed (i.e., the unshaded region is used). If no vertical shading is present, the full displayed velocity range is used.

(The complete figure set (7 images) is available.) 
Table 5

Fitted Parameters

\begin{tabular}{|c|c|c|c|c|c|c|c|c|c|c|}
\hline $\begin{array}{l}\text { Source } \\
\text { (name) }\end{array}$ & $\tau_{0}$ & $\begin{array}{c}\Delta v_{0} \\
\left(\mathrm{~km} \mathrm{~s}^{-1}\right)\end{array}$ & $\begin{array}{c}v_{0} \\
\left(\mathrm{~km} \mathrm{~s}^{-1}\right)\end{array}$ & $\begin{array}{l}T_{B, n} \\
(\mathrm{~K})\end{array}$ & $\begin{array}{c}\Delta v_{0, n} \\
\left(\mathrm{~km} \mathrm{~s}^{-1}\right)\end{array}$ & $\begin{array}{c}v_{0, n} \\
\left(\mathrm{~km} \mathrm{~s}^{-1}\right)\end{array}$ & $\begin{array}{c}T_{s} \\
(\mathrm{~K})\end{array}$ & $\begin{array}{l}N\left(\mathrm{H} \mathrm{I}_{\mathrm{abs}_{2}}\right. \\
\left(10^{20} \mathrm{~cm}^{-2}\right)\end{array}$ & 0 & $\mathscr{F}$ \\
\hline J0022 & $\begin{array}{l}0.018 \pm 0.001 \\
0.008 \pm 0.001 \\
0.009 \pm 0.001 \\
0.003 \pm 0.001\end{array}$ & $\begin{aligned} 2.8 & \pm 0.1 \\
10.3 & \pm 0.3 \\
1.7 & \pm 0.1 \\
1.0 & \pm 0.2\end{aligned}$ & $\begin{aligned} &-7.8 \pm 0.1 \\
&-4.3 \pm 0.2 \\
& 0.3 \pm 0.1 \\
&-14.6 \pm 0.1 \\
&\end{aligned}$ & $\begin{array}{c}1.5 \pm 0.0 \\
4.4 \pm 0.1 \\
0.8 \pm 0.0 \\
\ldots\end{array}$ & $\begin{aligned} & 3.1 \pm 0.1 \\
& 11.4 \pm 0.1 \\
& 1.8 \pm 0.1 \\
& \ldots\end{aligned}$ & $\begin{aligned} &-7.8 \pm 0.1 \\
&-4.1 \pm 0.1 \\
& 0.3 \pm 0.1 \\
& \cdots\end{aligned}$ & $\begin{aligned} & 83 \pm 2 \\
& 567 \pm 19 \\
& 84 \pm 4 \\
& \ldots\end{aligned}$ & $\begin{aligned} & 0.08 \pm 0.00 \\
& 0.88 \pm 0.05 \\
& 0.03 \pm 0.00 \\
& \ldots\end{aligned}$ & $\begin{array}{r}4 \\
4 \\
4 \\
\ldots\end{array}$ & 0.0 \\
\hline $3 \mathrm{C} 018 \mathrm{~A}$ & $\begin{array}{l}0.565 \pm 0.007 \\
0.134 \pm 0.003 \\
0.084 \pm 0.003 \\
0.007 \pm 0.003\end{array}$ & $\begin{array}{l}2.5 \pm 0.1 \\
5.5 \pm 0.2 \\
1.5 \pm 0.1 \\
0.7 \pm 0.3 \\
\end{array}$ & $\begin{array}{r}-9.1 \pm 0.1 \\
-6.2 \pm 0.1 \\
-5.0 \pm 0.1 \\
24.4 \pm 0.1 \\
\end{array}$ & $\begin{aligned} 6.9 & \pm 0.1 \\
25.2 & \pm 0.6 \\
& \ldots \\
& \ldots\end{aligned}$ & $\begin{array}{c}3.0 \pm 0.1 \\
6.0 \pm 0.1 \\
\ldots \\
\ldots\end{array}$ & $\begin{array}{c}-10.3 \pm 0.1 \\
-5.5 \pm 0.1 \\
\ldots \\
\ldots\end{array}$ & $\begin{array}{c}17 \pm 1 \\
196 \pm 5 \\
\ldots \\
\ldots\end{array}$ & $\begin{array}{c}0.48 \pm 0.04 \\
2.81 \pm 0.13 \\
\ldots \\
\ldots\end{array}$ & $\begin{array}{r}4 \\
2 \\
\cdots \\
\cdots\end{array}$ & 1.0 \\
\hline $3 \mathrm{C} 018 \mathrm{~B}$ & $\begin{array}{l}0.524 \pm 0.006 \\
0.149 \pm 0.005\end{array}$ & $\begin{array}{l}2.4 \pm 0.1 \\
6.2 \pm 0.1\end{array}$ & $\begin{array}{l}-9.0 \pm 0.1 \\
-6.8 \pm 0.1\end{array}$ & $\begin{array}{r}6.6 \pm 0.9 \\
22.5 \pm 0.5\end{array}$ & $\begin{array}{l}2.7 \pm 0.3 \\
7.0 \pm 0.1\end{array}$ & $\begin{array}{r}-10.3 \pm 0.3 \\
-6.7 \pm 0.1\end{array}$ & $\begin{array}{r}16 \pm 2 \\
162 \pm 4\end{array}$ & $\begin{array}{l}0.41 \pm 0.06 \\
2.89 \pm 0.10\end{array}$ & $\begin{array}{l}2 \\
1\end{array}$ & 1.0 \\
\hline $3 \mathrm{C} 041 \mathrm{~A}$ & $\begin{array}{l}0.033 \pm 0.004 \\
0.011 \pm 0.004 \\
0.029 \pm 0.003\end{array}$ & $\begin{array}{l}8.9 \pm 0.2 \\
1.2 \pm 0.2 \\
1.7 \pm 0.1\end{array}$ & $\begin{array}{r}-1.4 \pm 0.1 \\
1.8 \pm 0.1 \\
-30.5 \pm 0.1\end{array}$ & $\begin{aligned} & 11.4 \pm 0.2 \\
& 1.0 \pm 0.2 \\
& \ldots\end{aligned}$ & $\begin{array}{c}9.5 \pm 0.1 \\
1.3 \pm 0.1 \\
\ldots\end{array}$ & $\begin{aligned} &-1.4 \pm 0.1 \\
& 1.8 \pm 0.1 \\
& \cdots\end{aligned}$ & $\begin{array}{c}351 \pm 7 \\
98 \pm 16 \\
\ldots\end{array}$ & $\begin{array}{c}1.99 \pm 0.07 \\
0.02 \pm 0.01 \\
\ldots\end{array}$ & $\begin{array}{r}3 \\
3 \\
\cdots\end{array}$ & 1.0 \\
\hline $3 \mathrm{C} 041 \mathrm{~B}$ & $\begin{array}{l}0.022 \pm 0.005 \\
0.042 \pm 0.006 \\
0.023 \pm 0.005\end{array}$ & $\begin{array}{l}1.7 \pm 0.2 \\
7.1 \pm 0.2 \\
0.8 \pm 0.1\end{array}$ & $\begin{array}{r}-10.5 \pm 0.1 \\
-1.3 \pm 0.1 \\
-8.0 \pm 0.1\end{array}$ & $\begin{array}{c}0.3 \pm 0.0 \\
6.2 \pm 0.2 \\
\ldots\end{array}$ & $\begin{array}{c}1.8 \pm 0.1 \\
7.9 \pm 0.1 \\
\ldots\end{array}$ & $\begin{array}{c}-10.5 \pm 0.1 \\
-1.3 \pm 0.1 \\
\ldots\end{array}$ & $\begin{array}{r}14 \pm 3 \\
150 \pm 5 \\
\ldots\end{array}$ & $\begin{array}{c}0.01 \pm 0.00 \\
0.87 \pm 0.05 \\
\ldots\end{array}$ & $\begin{array}{r}3 \\
3 \\
\cdots\end{array}$ & 0.5 \\
\hline $3 \mathrm{C} 48$ & $\begin{array}{l}0.004 \pm 0.001 \\
0.008 \pm 0.001 \\
0.016 \pm 0.001 \\
0.004 \pm 0.001 \\
0.020 \pm 0.001 \\
0.040 \pm 0.001\end{array}$ & $\begin{array}{l}0.7 \pm 0.1 \\
5.4 \pm 0.3 \\
2.8 \pm 0.1 \\
1.3 \pm 0.2 \\
9.1 \pm 0.1 \\
2.3 \pm 0.1\end{array}$ & $\begin{array}{r}-55.1 \pm 0.1 \\
-15.6 \pm 0.1 \\
-11.0 \pm 0.1 \\
-5.0 \pm 0.1 \\
-2.1 \pm 0.1 \\
2.6 \pm 0.1\end{array}$ & $\begin{array}{l}0.1 \pm 0.0 \\
3.1 \pm 0.1 \\
1.3 \pm 0.0 \\
0.6 \pm 0.1 \\
6.7 \pm 0.1 \\
1.5 \pm 0.0\end{array}$ & $\begin{aligned} 0.7 & \pm 0.1 \\
6.0 & \pm 0.1 \\
3.1 & \pm 0.1 \\
1.4 & \pm 0.1 \\
10.1 & \pm 0.1 \\
2.3 & \pm 0.1\end{aligned}$ & $\begin{aligned}-52.8 & \pm 0.1 \\
-15.6 & \pm 0.1 \\
-11.6 & \pm 0.1 \\
-5.0 & \pm 0.1 \\
-2.1 & \pm 0.1 \\
2.8 & \pm 0.1\end{aligned}$ & $\begin{aligned} 25 & \pm 3 \\
406 & \pm 11 \\
79 & \pm 2 \\
169 & \pm 21 \\
346 & \pm 3 \\
38 & \pm 2\end{aligned}$ & $\begin{array}{l}0.00 \pm 0.00 \\
0.32 \pm 0.02 \\
0.07 \pm 0.00 \\
0.02 \pm 0.00 \\
1.20 \pm 0.02 \\
0.07 \pm 0.00\end{array}$ & $\begin{array}{l}6 \\
6 \\
6 \\
6 \\
6 \\
6\end{array}$ & 0.5 \\
\hline $4 \mathrm{C} 15.05$ & $\begin{array}{l}0.005 \pm 0.001 \\
0.057 \pm 0.001 \\
0.037 \pm 0.001 \\
0.048 \pm 0.001 \\
0.014 \pm 0.001 \\
0.001 \pm 0.001\end{array}$ & $\begin{array}{l}1.7 \pm 0.2 \\
2.6 \pm 0.1 \\
9.9 \pm 0.1 \\
2.7 \pm 0.1 \\
2.6 \pm 0.1 \\
5.0 \pm 1.1\end{array}$ & $\begin{array}{r}-13.9 \pm 0.1 \\
-10.5 \pm 0.1 \\
-7.3 \pm 0.1 \\
-5.4 \pm 0.1 \\
0.3 \pm 0.1 \\
6.7 \pm 0.5\end{array}$ & $\begin{aligned} 0.4 & \pm 0.0 \\
2.4 & \pm 0.0 \\
15.9 & \pm 0.4 \\
2.6 & \pm 0.0 \\
2.1 & \pm 0.1 \\
1.5 & \pm 0.3\end{aligned}$ & $\begin{aligned} 1.9 & \pm 0.1 \\
2.8 & \pm 0.1 \\
10.9 & \pm 0.1 \\
2.7 & \pm 0.1 \\
2.8 & \pm 0.1 \\
5.5 & \pm 0.1\end{aligned}$ & $\begin{aligned}-13.6 & \pm 0.1 \\
-11.3 & \pm 0.1 \\
-7.3 & \pm 0.1 \\
-5.3 & \pm 0.1 \\
0.3 & \pm 0.1 \\
6.7 & \pm 0.1\end{aligned}$ & $\begin{aligned} 82 & \pm 24 \\
46 & \pm 3 \\
438 & \pm 12 \\
59 & \pm 6 \\
145 & \pm 5 \\
1551 & \pm 298\end{aligned}$ & $\begin{array}{l}0.01 \pm 0.00 \\
0.13 \pm 0.01 \\
3.11 \pm 0.13 \\
0.15 \pm 0.02 \\
0.10 \pm 0.01 \\
0.14 \pm 0.05\end{array}$ & $\begin{array}{l}6 \\
2 \\
1 \\
6 \\
6 \\
6\end{array}$ & 0.0 \\
\hline $3 \mathrm{C} 78$ & $\begin{array}{l}0.147 \pm 0.004 \\
0.029 \pm 0.004 \\
0.196 \pm 0.004 \\
1.369 \pm 0.006 \\
0.089 \pm 0.005 \\
0.054 \pm 0.006\end{array}$ & $\begin{aligned} 3.1 & \pm 0.1 \\
14.9 & \pm 0.6 \\
1.9 & \pm 0.1 \\
2.2 & \pm 0.1 \\
4.1 & \pm 0.1 \\
1.3 & \pm 0.1\end{aligned}$ & $\begin{aligned}-7.8 & \pm 0.1 \\
-7.5 & \pm 0.2 \\
4.2 & \pm 0.1 \\
6.9 & \pm 0.1 \\
11.1 & \pm 0.1 \\
10.4 & \pm 0.1\end{aligned}$ & $\begin{aligned} 8.7 & \pm 0.1 \\
10.8 & \pm 0.5 \\
7.2 & \pm 0.1 \\
24.1 & \pm 0.1 \\
14.6 & \pm 0.8 \\
& \cdots\end{aligned}$ & $\begin{aligned} 3.1 & \pm 0.1 \\
15.6 & \pm 0.1 \\
2.1 & \pm 0.1 \\
2.4 & \pm 0.1 \\
4.6 & \pm 0.1 \\
& \cdots\end{aligned}$ & $\begin{aligned}-7.8 & \pm 0.1 \\
-7.5 & \pm 0.1 \\
4.0 & \pm 0.1 \\
7.0 & \pm 0.1 \\
11.4 & \pm 0.1 \\
\cdots & \end{aligned}$ & $\begin{aligned} & 65 \pm 3 \\
& 385 \pm 18 \\
& 39 \pm 1 \\
& 39 \pm 7 \\
& 175 \pm 10 \\
& \cdots\end{aligned}$ & $\begin{aligned} & 0.57 \pm 0.03 \\
& 3.17 \pm 0.25 \\
& 0.29 \pm 0.01 \\
& 2.32 \pm 0.43 \\
& 1.25 \pm 0.11 \\
& \ldots\end{aligned}$ & $\begin{array}{c}6 \\
6 \\
1 \\
2 \\
6 \\
\cdots\end{array}$ & 1.0 \\
\hline 4C16.09 & $\begin{array}{l}0.014 \pm 0.001 \\
0.436 \pm 0.002 \\
0.227 \pm 0.004 \\
0.086 \pm 0.001 \\
0.042 \pm 0.001 \\
0.013 \pm 0.001\end{array}$ & $\begin{array}{l}1.3 \pm 0.1 \\
4.2 \pm 0.1 \\
1.8 \pm 0.1 \\
5.4 \pm 0.1 \\
1.8 \pm 0.1 \\
4.1 \pm 0.2\end{array}$ & $\begin{aligned}-9.7 & \pm 0.1 \\
-1.0 & \pm 0.1 \\
0.8 & \pm 0.1 \\
5.9 & \pm 0.1 \\
7.6 & \pm 0.1 \\
-8.7 & \pm 0.1\end{aligned}$ & $\begin{aligned} 0.3 & \pm 0.0 \\
35.6 & \pm 0.2 \\
6.5 & \pm 0.2 \\
21.4 & \pm 0.2 \\
4.4 & \pm 0.2 \\
& \ldots\end{aligned}$ & $\begin{array}{l}1.6 \pm 0.1 \\
4.1 \pm 0.1 \\
1.8 \pm 0.1 \\
5.9 \pm 0.1 \\
1.9 \pm 0.1 \\
\cdots\end{array}$ & $\begin{aligned}-11.7 & \pm 0.1 \\
-1.0 & \pm 0.1 \\
0.9 & \pm 0.1 \\
5.7 & \pm 0.1 \\
7.5 & \pm 0.1 \\
\ldots & \end{aligned}$ & $\begin{aligned} & 25 \pm 4 \\
& 106 \pm 3 \\
& 54 \pm 10 \\
& 260 \pm 3 \\
& 120 \pm 8 \\
& \ldots\end{aligned}$ & $\begin{aligned} 0.01 & \pm 0.00 \\
3.74 & \pm 0.13 \\
0.44 & \pm 0.08 \\
2.37 & \pm 0.05 \\
0.17 & \pm 0.01 \\
\ldots & \end{aligned}$ & $\begin{array}{c}6 \\
6 \\
6 \\
6 \\
6 \\
\cdots\end{array}$ & 1.0 \\
\hline $3 \mathrm{C} 111 \mathrm{~A}$ & $\begin{array}{l}0.013 \pm 0.003 \\
0.041 \pm 0.003 \\
0.322 \pm 0.004 \\
0.172 \pm 0.005 \\
0.201 \pm 0.011 \\
0.790 \pm 0.009 \\
0.643 \pm 0.020 \\
0.232 \pm 0.004 \\
0.037 \pm 0.003 \\
0.025 \pm 20000.0 \\
0.114 \pm 0.006 \\
0.069 \pm 0.004\end{array}$ & $\begin{array}{l}5.7 \pm 1.0 \\
2.6 \pm 0.1 \\
2.6 \pm 0.1 \\
8.6 \pm 0.3 \\
1.5 \pm 0.1 \\
3.9 \pm 0.1 \\
3.3 \pm 0.1 \\
5.2 \pm 0.1 \\
1.7 \pm 0.1 \\
0.1 \pm 10000.0 \\
2.1 \pm 0.1 \\
1.3 \pm 0.1\end{array}$ & $\begin{aligned}-28.9 & \pm 0.4 \\
-21.9 & \pm 0.1 \\
-16.8 & \pm 0.1 \\
-9.1 & \pm 0.2 \\
-4.7 & \pm 0.1 \\
-1.8 & \pm 0.1 \\
1.8 & \pm 0.1 \\
6.1 & \pm 0.1 \\
-32.4 & \pm 0.1 \\
6.6 & \pm 900.0 \\
-8.3 & \pm 0.1 \\
8.4 & \pm 0.1\end{aligned}$ & $\begin{aligned} & 6.3 \pm 0.5 \\
& 5.4 \pm 0.2 \\
& 8.5 \pm 0.1 \\
& 40.7 \pm 1.3 \\
& 12.2 \pm 0.7 \\
& 39.3 \pm 0.7 \\
& 41.0 \pm 1.9 \\
& 26.8 \pm 0.4 \\
& \ldots \\
& \ldots \\
& \ldots \\
& \ldots\end{aligned}$ & $\begin{aligned} & 6.2 \pm 0.1 \\
& 2.8 \pm 0.1 \\
& 2.9 \pm 0.1 \\
& 9.5 \pm 0.1 \\
& 1.6 \pm 0.1 \\
& 4.1 \pm 0.1 \\
& 3.6 \pm 0.1 \\
& 5.7 \pm 0.1 \\
& \ldots \\
& \ldots \\
& \ldots \\
& \ldots\end{aligned}$ & $\begin{aligned}-27.0 & \pm 0.1 \\
-20.5 & \pm 0.1 \\
-16.6 & \pm 0.1 \\
-9.0 & \pm 0.1 \\
-4.6 & \pm 0.1 \\
-1.6 & \pm 0.1 \\
1.8 & \pm 0.1 \\
6.0 & \pm 0.1 \\
\ldots & \\
\ldots & \\
\ldots & \end{aligned}$ & $\begin{aligned} & 487 \pm 44 \\
& 131 \pm 6 \\
& 34 \pm 5 \\
& 248 \pm 10 \\
& 65 \pm 15 \\
& 77 \pm 4 \\
& 93 \pm 8 \\
& 129 \pm 1 \\
& \ldots \\
& \ldots \\
& \ldots \\
& \ldots\end{aligned}$ & $\begin{aligned} & 0.69 \pm 0.15 \\
& 0.27 \pm 0.02 \\
& 0.56 \pm 0.10 \\
& 7.18 \pm 0.47 \\
& 0.38 \pm 0.10 \\
& 4.57 \pm 0.32 \\
& 3.85 \pm 0.38 \\
& 3.02 \pm 0.10 \\
& \ldots \\
& \ldots \\
& \ldots \\
& \ldots\end{aligned}$ & $\begin{array}{r}3 \\
1 \\
5 \\
12 \\
2 \\
4 \\
12 \\
12 \\
\ldots \\
\ldots \\
\ldots \\
\ldots\end{array}$ & 1.0 \\
\hline $3 \mathrm{C} 111 \mathrm{~B}$ & $\begin{array}{l}0.019 \pm 0.002 \\
0.111 \pm 0.003 \\
0.092 \pm 0.004 \\
0.400 \pm 0.005 \\
0.967 \pm 0.020\end{array}$ & $\begin{aligned} 1.7 & \pm 0.1 \\
2.7 & \pm 0.1 \\
12.1 & \pm 0.3 \\
2.8 & \pm 0.1 \\
3.7 & \pm 0.1\end{aligned}$ & $\begin{array}{r}-54.8 \pm 0.1 \\
-16.8 \pm 0.1 \\
-10.8 \pm 0.3 \\
-8.3 \pm 0.1 \\
-1.7 \pm 0.1\end{array}$ & $\begin{aligned} 0.5 & \pm 0.0 \\
2.8 & \pm 0.1 \\
35.9 & \pm 1.0 \\
3.7 & \pm 0.1 \\
29.2 & \pm 1.1\end{aligned}$ & $\begin{aligned} 1.7 & \pm 0.1 \\
3.0 & \pm 0.1 \\
12.7 & \pm 0.1 \\
3.1 & \pm 0.1 \\
3.9 & \pm 0.1\end{aligned}$ & $\begin{aligned}-56.2 & \pm 0.1 \\
-16.7 & \pm 0.1 \\
-10.8 & \pm 0.1 \\
-8.3 & \pm 0.1 \\
-1.7 & \pm 0.1\end{aligned}$ & $\begin{aligned} 26 & \pm 1 \\
30 & \pm 5 \\
409 & \pm 10 \\
14 & \pm 8 \\
56 & \pm 5\end{aligned}$ & $\begin{array}{l}0.02 \pm 0.00 \\
0.17 \pm 0.03 \\
8.86 \pm 0.42 \\
0.32 \pm 0.20 \\
3.88 \pm 0.43\end{array}$ & $\begin{array}{r}10 \\
1 \\
10 \\
10 \\
10\end{array}$ & 1.0 \\
\hline
\end{tabular}


Table 5

(Continued)

\begin{tabular}{|c|c|c|c|c|c|c|c|c|c|c|}
\hline $\begin{array}{l}\text { Source } \\
\text { (name) }\end{array}$ & $\tau_{0}$ & $\begin{array}{c}\Delta v_{0} \\
\left(\mathrm{~km} \mathrm{~s}^{-1}\right)\end{array}$ & $\begin{array}{c}v_{0} \\
\left(\mathrm{~km} \mathrm{~s}^{-1}\right)\end{array}$ & $\begin{array}{l}T_{B, n} \\
(\mathrm{~K})\end{array}$ & $\begin{array}{c}\Delta v_{0, n} \\
\left(\mathrm{~km} \mathrm{~s}^{-1}\right)\end{array}$ & $\begin{array}{c}v_{0, n} \\
\left(\mathrm{~km} \mathrm{~s}^{-1}\right)\end{array}$ & $\begin{array}{c}T_{s} \\
(\mathrm{~K})\end{array}$ & $\begin{array}{l}N(\mathrm{H} \mathrm{I})_{\mathrm{abs}} \\
\left(10^{20} \mathrm{~cm}^{-2}\right)\end{array}$ & (1) & $\mathscr{\mathscr { F }}$ \\
\hline & $0.347 \pm 0.054$ & $2.7 \pm 0.1$ & $2.1 \pm 0.1$ & $11.1 \pm 3.1$ & $2.7 \pm 0.1$ & $2.1 \pm 0.1$ & $58 \pm 13$ & $1.07 \pm 0.31$ & 2 & \\
\hline & $0.342 \pm 0.032$ & $5.6 \pm 0.4$ & $3.7 \pm 0.4$ & $33.8 \pm 3.8$ & $6.2 \pm 0.1$ & $3.9 \pm 0.1$ & $120 \pm 12$ & $4.51 \pm 0.71$ & 10 & \\
\hline & $0.181 \pm 0.006$ & $0.8 \pm 0.1$ & $-2.3 \pm 0.1$ & $\ldots$ & $\ldots$ & $\ldots$ & $\ldots$ & $\ldots$ & $\ldots$ & \\
\hline & $0.015 \pm 0.002$ & $2.2 \pm 0.2$ & $-51.3 \pm 0.1$ & $\ldots$ & $\ldots$ & $\ldots$ & $\ldots$ & $\ldots$ & $\ldots$ & \\
\hline & $0.212 \pm 0.005$ & $2.1 \pm 0.1$ & $8.0 \pm 0.1$ & $\cdots$ & $\cdots$ & $\cdots$ & $\cdots$ & $\cdots$ & $\cdots$ & \\
\hline \multirow[t]{11}{*}{$3 \mathrm{C} 111 \mathrm{C}$} & $0.027 \pm 0.004$ & $2.4 \pm 0.2$ & $-31.4 \pm 0.1$ & $0.9 \pm 0.1$ & $2.6 \pm 0.1$ & $-31.4 \pm 0.1$ & $35 \pm 3$ & $0.04 \pm 0.01$ & 11 & 1.0 \\
\hline & $0.029 \pm 0.004$ & $2.6 \pm 0.2$ & $-27.8 \pm 0.1$ & $1.1 \pm 0.1$ & $2.9 \pm 0.1$ & $-26.0 \pm 0.1$ & $39 \pm 2$ & $0.06 \pm 0.01$ & 11 & \\
\hline & $0.282 \pm 0.045$ & $2.1 \pm 0.1$ & $-16.9 \pm 0.1$ & $3.9 \pm 0.9$ & $2.1 \pm 0.1$ & $-17.8 \pm 0.1$ & $20 \pm 4$ & $0.23 \pm 0.07$ & 11 & \\
\hline & $0.088 \pm 0.027$ & $2.5 \pm 0.6$ & $-15.3 \pm 0.5$ & $8.4 \pm 2.4$ & $2.8 \pm 0.1$ & $-14.1 \pm 0.1$ & $90 \pm 34$ & $0.39 \pm 0.22$ & 1 & \\
\hline & $0.182 \pm 0.011$ & $11.2 \pm 0.6$ & $-8.1 \pm 0.6$ & $17.8 \pm 1.3$ & $12.4 \pm 0.1$ & $-8.1 \pm 0.1$ & $118 \pm 28$ & $4.69 \pm 1.20$ & 11 & \\
\hline & $0.816 \pm 0.050$ & $2.3 \pm 0.1$ & $-2.1 \pm 0.1$ & $3.5 \pm 1.0$ & $2.3 \pm 0.1$ & $-2.4 \pm 0.1$ & $20 \pm 11$ & $0.75 \pm 0.44$ & 11 & \\
\hline & $0.456 \pm 0.044$ & $7.3 \pm 0.3$ & $1.6 \pm 0.4$ & $34.6 \pm 4.7$ & $8.1 \pm 0.1$ & $1.6 \pm 0.1$ & $108 \pm 18$ & $7.00 \pm 1.41$ & 11 & \\
\hline & $0.584 \pm 0.043$ & $2.5 \pm 0.1$ & $1.1 \pm 0.1$ & $\ldots$ & $\ldots$ & $\ldots$ & $\ldots$ & $\ldots$ & $\ldots$ & \\
\hline & $0.416 \pm 0.008$ & $1.6 \pm 0.1$ & $-8.4 \pm 0.1$ & $\cdots$ & $\cdots$ & $\cdots$ & $\cdots$ & $\cdots$ & $\cdots$ & \\
\hline & $0.211 \pm 0.014$ & $1.5 \pm 0.1$ & $-5.5 \pm 0.1$ & $\cdots$ & $\cdots$ & $\cdots$ & $\cdots$ & $\cdots$ & $\cdots$ & \\
\hline & $0.058 \pm 0.007$ & $1.8 \pm 0.2$ & $6.3 \pm 0.1$ & $\cdots$ & $\cdots$ & $\cdots$ & $\cdots$ & $\cdots$ & $\cdots$ & \\
\hline \multirow[t]{3}{*}{$3 \mathrm{C} 120$} & $0.617 \pm 0.005$ & $1.3 \pm 0.1$ & $6.1 \pm 0.1$ & $7.7 \pm 0.1$ & $1.3 \pm 0.1$ & $5.5 \pm 0.1$ & $20 \pm 9$ & $0.31 \pm 0.15$ & 2 & 1.0 \\
\hline & $1.681 \pm 0.004$ & $4.4 \pm 0.1$ & $7.3 \pm 0.1$ & $36.6 \pm 0.2$ & $4.9 \pm 0.1$ & $6.6 \pm 0.1$ & $44 \pm 4$ & $6.35 \pm 0.60$ & 1 & \\
\hline & $0.776 \pm 0.012$ & $2.3 \pm 0.1$ & $9.9 \pm 0.1$ & $12.7 \pm 0.3$ & $2.5 \pm 0.1$ & $10.6 \pm 0.1$ & $23 \pm 5$ & $0.82 \pm 0.18$ & 3 & \\
\hline \multirow[t]{7}{*}{$3 \mathrm{C} 123 \mathrm{~A}$} & $0.064 \pm 0.001$ & $3.1 \pm 0.1$ & $-19.9 \pm 0.1$ & $1.5 \pm 0.0$ & $3.8 \pm 0.1$ & $-21.1 \pm 0.1$ & $26 \pm 2$ & $0.10 \pm 0.01$ & 7 & 1.0 \\
\hline & $0.379 \pm 0.035$ & $2.2 \pm 0.1$ & $1.6 \pm 0.1$ & $3.2 \pm 0.7$ & $2.2 \pm 0.1$ & $1.9 \pm 0.1$ & $19 \pm 14$ & $0.32 \pm 0.23$ & 7 & \\
\hline & $0.044 \pm 0.004$ & $10.1 \pm 0.3$ & $3.7 \pm 0.1$ & $27.0 \pm 2.8$ & $12.2 \pm 0.1$ & $3.9 \pm 0.1$ & $619 \pm 79$ & $5.36 \pm 0.91$ & 7 & \\
\hline & $0.810 \pm 0.071$ & $4.4 \pm 0.1$ & $5.3 \pm 0.2$ & $23.1 \pm 4.6$ & $5.3 \pm 0.1$ & $5.2 \pm 0.1$ & $67 \pm 25$ & $4.67 \pm 1.86$ & 7 & \\
\hline & $0.628 \pm 0.037$ & $1.8 \pm 0.1$ & $5.5 \pm 0.1$ & $4.3 \pm 0.7$ & $1.7 \pm 0.1$ & $6.3 \pm 0.1$ & $18 \pm 11$ & $0.40 \pm 0.25$ & 7 & \\
\hline & $0.008 \pm 0.001$ & $4.0 \pm 0.3$ & $20.2 \pm 0.1$ & $1.0 \pm 0.1$ & $4.0 \pm 0.1$ & $17.9 \pm 0.1$ & $124 \pm 9$ & $0.08 \pm 0.01$ & 7 & \\
\hline & $1.020 \pm 0.096$ & $2.3 \pm 0.1$ & $3.7 \pm 0.1$ & $\ldots$ & $\ldots$ & . & $\ldots$ & $\ldots$ & $\cdots$ & \\
\hline \multirow[t]{6}{*}{$3 \mathrm{C} 123 \mathrm{~B}$} & $0.064 \pm 0.001$ & $3.2 \pm 0.1$ & $-19.3 \pm 0.1$ & $0.9 \pm 0.0$ & $3.6 \pm 0.1$ & $-20.4 \pm 0.1$ & $16 \pm 2$ & $0.07 \pm 0.01$ & 6 & 1.0 \\
\hline & $1.648 \pm 0.005$ & $4.3 \pm 0.1$ & $4.4 \pm 0.1$ & $17.6 \pm 0.2$ & $4.8 \pm 0.1$ & $4.4 \pm 0.1$ & $36 \pm 14$ & $5.08 \pm 1.98$ & 3 & \\
\hline & $0.042 \pm 0.003$ & $10.9 \pm 0.3$ & $4.9 \pm 0.1$ & $27.8 \pm 2.2$ & $10.9 \pm 0.1$ & $4.5 \pm 0.1$ & $664 \pm 52$ & $5.98 \pm 0.71$ & 2 & \\
\hline & $0.253 \pm 0.007$ & $1.5 \pm 0.1$ & $5.5 \pm 0.1$ & $5.4 \pm 0.6$ & $1.6 \pm 0.1$ & $6.0 \pm 0.1$ & $81 \pm 43$ & $0.59 \pm 0.32$ & 6 & \\
\hline & $0.004 \pm 0.001$ & $3.2 \pm 0.5$ & $20.1 \pm 0.2$ & $1.4 \pm 0.2$ & $3.5 \pm 0.1$ & $18.3 \pm 0.1$ & $334 \pm 52$ & $0.09 \pm 0.02$ & 1 & \\
\hline & $0.064 \pm 0.003$ & $1.7 \pm 0.1$ & $8.5 \pm 0.1$ & $\cdots$ & . & .. & $\cdots$ & $\ldots$ & $\cdots$ & \\
\hline \multirow[t]{7}{*}{$3 \mathrm{C} 132$} & $0.197 \pm 0.009$ & $7.2 \pm 0.1$ & $1.7 \pm 0.1$ & $27.8 \pm 1.5$ & $7.2 \pm 0.1$ & $1.9 \pm 0.1$ & $165 \pm 12$ & $4.57 \pm 0.41$ & 7 & 1.0 \\
\hline & $1.472 \pm 0.005$ & $2.0 \pm 0.1$ & $7.8 \pm 0.9$ & $7.1 \pm 0.1$ & $2.0 \pm 0.1$ & $7.8 \pm 0.1$ & $27 \pm 19$ & $1.55 \pm 1.12$ & 4 & \\
\hline & $0.230 \pm 0.003$ & $6.7 \pm 0.1$ & $12.1 \pm 0.1$ & $16.2 \pm 0.1$ & $7.3 \pm 0.1$ & $11.4 \pm 0.1$ & $80 \pm 4$ & $2.39 \pm 0.15$ & 3 & \\
\hline & $0.129 \pm 0.022$ & $1.8 \pm 0.1$ & $1.7 \pm 99$ & $\ldots$ & $\ldots$ & $\ldots$ & $\ldots$ & $\ldots$ & $\cdots$ & \\
\hline & $0.273 \pm 0.010$ & $1.5 \pm 0.1$ & $9.4 \pm 0.1$ & $\cdots$ & $\cdots$ & $\cdots$ & $\cdots$ & $\cdots$ & $\cdots$ & \\
\hline & $0.059 \pm 0.004$ & $2.4 \pm 0.1$ & $14.8 \pm 0.1$ & $\cdots$ & $\cdots$ & $\ldots$ & $\cdots$ & $\cdots$ & $\ldots$ & \\
\hline & $0.042 \pm 0.017$ & $3.4 \pm 0.1$ & $2.2 \pm 0.1$ & $\cdots$ & $\cdots$ & $\cdots$ & $\cdots$ & $\cdots$ & $\cdots$ & \\
\hline \multirow[t]{8}{*}{$3 C 133$} & $0.033 \pm 0.005$ & $9.5 \pm 0.3$ & $-29.8 \pm 0.1$ & $17.2 \pm 0.9$ & $10.4 \pm 0.1$ & $-28.8 \pm 0.1$ & $537 \pm 28$ & $3.26 \pm 0.26$ & 8 & 1.0 \\
\hline & $0.134 \pm 0.007$ & $2.4 \pm 0.1$ & $-1.5 \pm 0.1$ & $2.1 \pm 0.1$ & $2.7 \pm 0.1$ & $-1.6 \pm 0.1$ & $26 \pm 16$ & $0.17 \pm 0.11$ & 2 & \\
\hline & $0.240 \pm 0.011$ & $9.6 \pm 0.1$ & $3.1 \pm 0.1$ & $59.2 \pm 3.0$ & $9.6 \pm 0.1$ & $2.8 \pm 0.1$ & $283 \pm 13$ & $12.67 \pm 0.87$ & 1 & \\
\hline & $0.706 \pm 0.010$ & $2.7 \pm 0.1$ & $3.7 \pm 0.1$ & $2.7 \pm 0.1$ & $3.0 \pm 0.1$ & $3.4 \pm 0.1$ & $11 \pm 15$ & $0.44 \pm 0.61$ & 8 & \\
\hline & $0.845 \pm 0.029$ & $1.5 \pm 0.1$ & $7.6 \pm 0.1$ & $1.6 \pm 0.7$ & $1.5 \pm 0.1$ & $7.6 \pm 0.1$ & $23 \pm 19$ & $0.58 \pm 0.48$ & 8 & \\
\hline & $0.780 \pm 0.022$ & $2.6 \pm 0.1$ & $8.4 \pm 0.1$ & $18.0 \pm 1.0$ & $2.9 \pm 0.1$ & $8.7 \pm 0.1$ & $46 \pm 9$ & $1.82 \pm 0.37$ & 8 & \\
\hline & $0.084 \pm 0.005$ & $3.2 \pm 0.1$ & $-27.8 \pm 0.1$ & $\ldots$ & $\ldots$ & $\ldots$ & $\ldots$ & $\ldots$ & $\ldots$ & \\
\hline & $0.077 \pm 0.005$ & $1.6 \pm 0.1$ & $-4.2 \pm 0.1$ & $\cdots$ & $\cdots$ & $\cdots$ & $\cdots$ & $\cdots$ & $\cdots$ & \\
\hline \multirow[t]{8}{*}{$3 \mathrm{C} 138$} & $0.033 \pm 0.001$ & $2.3 \pm 0.1$ & $-22.0 \pm 0.1$ & $1.9 \pm 0.1$ & $2.5 \pm 0.1$ & $-21.2 \pm 0.1$ & $60 \pm 2$ & $0.09 \pm 0.01$ & 8 & 1.0 \\
\hline & $0.026 \pm 0.002$ & $4.3 \pm 0.2$ & $-6.7 \pm 0.1$ & $7.7 \pm 0.3$ & $4.5 \pm 0.1$ & $-6.7 \pm 0.1$ & $297 \pm 11$ & $0.65 \pm 0.05$ & 8 & \\
\hline & $0.322 \pm 0.003$ & $4.3 \pm 0.1$ & $0.0 \pm 0.1$ & $33.4 \pm 0.2$ & $4.8 \pm 0.1$ & $0.0 \pm 0.1$ & $125 \pm 3$ & $3.39 \pm 0.09$ & 8 & \\
\hline & $0.079 \pm 0.003$ & $1.2 \pm 0.1$ & $1.6 \pm 0.1$ & $2.0 \pm 0.2$ & $1.3 \pm 0.1$ & $1.6 \pm 0.1$ & $47 \pm 19$ & $0.08 \pm 0.04$ & 8 & \\
\hline & $0.003 \pm 0.003$ & $37.4 \pm 4.9$ & $2.9 \pm 1.5$ & $4.8 \pm 1.1$ & $33.7 \pm 0.1$ & $2.6 \pm 0.1$ & $1427 \pm 302$ & $3.52 \pm 1.23$ & 8 & \\
\hline & $1.057 \pm 0.005$ & $2.4 \pm 0.1$ & $6.3 \pm 0.1$ & $20.6 \pm 0.2$ & $2.6 \pm 0.1$ & $5.9 \pm 0.1$ & $39 \pm 7$ & $1.92 \pm 0.38$ & 8 & \\
\hline & $0.432 \pm 0.003$ & $3.0 \pm 0.1$ & $9.0 \pm 0.1$ & $23.5 \pm 0.2$ & $3.3 \pm 0.1$ & $9.5 \pm 0.1$ & $66 \pm 1$ & $1.68 \pm 0.04$ & 8 & \\
\hline & $0.011 \pm 0.002$ & $2.7 \pm 0.5$ & $13.3 \pm 0.2$ & $4.2 \pm 0.5$ & $3.0 \pm 0.1$ & $12.0 \pm 0.1$ & $422 \pm 55$ & $0.24 \pm 0.06$ & 8 & \\
\hline \multirow[t]{3}{*}{ PKS0531 } & $0.001 \pm 0.001$ & $12.1 \pm 1.5$ & $-24.3 \pm 0.5$ & $2.9 \pm 0.5$ & $13.3 \pm 0.1$ & $-24.3 \pm 0.1$ & $2049 \pm 400$ & $0.68 \pm 0.20$ & 10 & 1.0 \\
\hline & $0.007 \pm 0.001$ & $3.5 \pm 0.2$ & $-23.0 \pm 0.1$ & $0.1 \pm 0.0$ & $3.8 \pm 0.1$ & $-20.7 \pm 0.1$ & $24 \pm 4$ & $0.01 \pm 0.00$ & 10 & \\
\hline & $0.006 \pm 0.001$ & $6.2 \pm 0.3$ & $-9.0 \pm 0.1$ & $0.8 \pm 0.0$ & $6.8 \pm 0.1$ & $-9.0 \pm 0.1$ & $240 \pm 130$ & $0.16 \pm 0.09$ & 1 & \\
\hline
\end{tabular}


Table 5

(Continued)

\begin{tabular}{|c|c|c|c|c|c|c|c|c|c|c|}
\hline $\begin{array}{l}\text { Source } \\
\text { (name) }\end{array}$ & $\tau_{0}$ & $\begin{array}{c}\Delta v_{0} \\
\left(\mathrm{~km} \mathrm{~s}^{-1}\right)\end{array}$ & $\begin{array}{c}v_{0} \\
\left(\mathrm{~km} \mathrm{~s}^{-1}\right)\end{array}$ & $\begin{array}{l}T_{B, n} \\
(\mathrm{~K})\end{array}$ & $\begin{array}{c}\Delta v_{0, n} \\
\left(\mathrm{~km} \mathrm{~s}^{-1}\right)\end{array}$ & $\begin{array}{c}v_{0, n} \\
\left(\mathrm{~km} \mathrm{~s}^{-1}\right)\end{array}$ & $\begin{array}{c}T_{s} \\
(\mathrm{~K})\end{array}$ & $\begin{array}{l}N\left(\mathrm{H} \mathrm{I}_{\mathrm{abs}}\right. \\
\left(10^{20} \mathrm{~cm}^{-2}\right)\end{array}$ & O & $\mathscr{F}$ \\
\hline & $0.403 \pm 0.009$ & $1.9 \pm 0.1$ & $1.6 \pm 0.1$ & $3.7 \pm 0.3$ & $1.9 \pm 0.1$ & $1.5 \pm 0.1$ & $28 \pm 26$ & $0.44 \pm 0.40$ & 3 & \\
\hline & $0.141 \pm 0.005$ & $9.7 \pm 0.1$ & $4.6 \pm 0.1$ & $69.4 \pm 2.7$ & $10.3 \pm 0.1$ & $4.3 \pm 0.1$ & $542 \pm 25$ & $14.48 \pm 0.89$ & 2 & \\
\hline & $0.190 \pm 0.003$ & $2.4 \pm 0.1$ & $9.5 \pm 0.1$ & $14.9 \pm 0.3$ & $2.6 \pm 0.1$ & $10.0 \pm 0.1$ & $100 \pm 10$ & $0.88 \pm 0.09$ & 10 & \\
\hline & $0.019 \pm 0.001$ & $2.3 \pm 0.2$ & $12.1 \pm 0.1$ & $3.0 \pm 0.2$ & $2.5 \pm 0.1$ & $12.1 \pm 0.1$ & $132 \pm 31$ & $0.11 \pm 0.03$ & 10 & \\
\hline & $0.005 \pm 0.001$ & $4.1 \pm 0.2$ & $21.0 \pm 0.1$ & $2.0 \pm 0.1$ & $4.5 \pm 0.1$ & $20.9 \pm 0.1$ & $380 \pm 14$ & $0.16 \pm 0.01$ & 10 & \\
\hline & $0.075 \pm 0.005$ & $2.7 \pm 0.1$ & $6.0 \pm 0.1$ & $\ldots$ & $\ldots$ & $\ldots$ & $\ldots$ & $\ldots$ & $\ldots$ & \\
\hline & $0.088 \pm 0.007$ & $1.9 \pm 0.1$ & $3.2 \pm 0.1$ & $\cdots$ & $\cdots$ & $\cdots$ & $\cdots$ & $\cdots$ & $\cdots$ & \\
\hline \multirow[t]{9}{*}{$3 \mathrm{C} 147$} & $0.013 \pm 0.001$ & $2.0 \pm 0.1$ & $-19.4 \pm 0.1$ & $0.8 \pm 0.0$ & $2.2 \pm 0.1$ & $-19.4 \pm 0.1$ & $65 \pm 5$ & $0.03 \pm 0.00$ & 9 & 1.0 \\
\hline & $0.054 \pm 0.009$ & $1.9 \pm 0.1$ & $-13.7 \pm 0.1$ & $2.0 \pm 0.3$ & $2.0 \pm 0.1$ & $-15.0 \pm 0.1$ & $36 \pm 8$ & $0.07 \pm 0.02$ & 9 & \\
\hline & $0.120 \pm 0.021$ & $6.4 \pm 0.3$ & $-11.1 \pm 0.1$ & $23.9 \pm 4.5$ & $6.6 \pm 0.1$ & $-11.2 \pm 0.1$ & $218 \pm 37$ & $3.25 \pm 0.82$ & 9 & \\
\hline & $0.165 \pm 0.020$ & $2.3 \pm 0.1$ & $-10.7 \pm 0.1$ & $5.0 \pm 0.7$ & $2.1 \pm 0.1$ & $-9.9 \pm 0.1$ & $37 \pm 6$ & $0.27 \pm 0.06$ & 9 & \\
\hline & $0.278 \pm 0.004$ & $5.9 \pm 0.1$ & $0.5 \pm 0.1$ & $23.6 \pm 0.4$ & $6.5 \pm 0.1$ & $0.6 \pm 0.1$ & $109 \pm 9$ & $3.49 \pm 0.33$ & 9 & \\
\hline & $0.035 \pm 0.001$ & $1.6 \pm 0.1$ & $5.1 \pm 0.1$ & $0.9 \pm 0.0$ & $1.8 \pm 0.1$ & $5.6 \pm 0.1$ & $25 \pm 3$ & $0.03 \pm 0.00$ & 9 & \\
\hline & $0.725 \pm 0.006$ & $1.7 \pm 0.1$ & $-8.0 \pm 0.1$ & $\ldots$ & $\ldots$ & $\ldots$ & $\ldots$ & $\ldots$ & $\ldots$ & \\
\hline & $0.208 \pm 0.004$ & $2.0 \pm 0.1$ & $-0.1 \pm 0.1$ & $\cdots$ & $\cdots$ & $\cdots$ & $\cdots$ & $\ldots$ & $\cdots$ & \\
\hline & $0.042 \pm 0.003$ & $1.5 \pm 0.1$ & $1.8 \pm 0.1$ & $\cdots$ & $\cdots$ & $\cdots$ & $\cdots$ & $\cdots$ & $\cdots$ & \\
\hline \multirow[t]{8}{*}{$3 \mathrm{C} 154$} & $0.061 \pm 0.005$ & $3.1 \pm 0.2$ & $-23.2 \pm 0.1$ & $1.6 \pm 0.1$ & $3.4 \pm 0.1$ & $-23.1 \pm 0.1$ & $25 \pm 3$ & $0.10 \pm 0.02$ & 5 & 1.0 \\
\hline & $0.012 \pm 0.005$ & $7.4 \pm 1.4$ & $-22.9 \pm 0.3$ & $7.2 \pm 3.0$ & $7.4 \pm 0.1$ & $-22.4 \pm 0.1$ & $587 \pm 240$ & $1.05 \pm 0.65$ & 6 & \\
\hline & $0.123 \pm 0.020$ & $14.0 \pm 0.5$ & $0.6 \pm 0.2$ & $87.5 \pm 14.8$ & $15.0 \pm 0.1$ & $0.7 \pm 0.1$ & $762 \pm 130$ & $25.55 \pm 6.15$ & 2 & \\
\hline & $1.300 \pm 0.015$ & $2.3 \pm 0.1$ & $2.0 \pm 0.1$ & $4.2 \pm 0.2$ & $2.2 \pm 0.1$ & $2.0 \pm 0.1$ & $10 \pm 15$ & $0.62 \pm 0.91$ & 4 & \\
\hline & $0.733 \pm 0.016$ & $3.9 \pm 0.1$ & $4.8 \pm 0.1$ & $7.0 \pm 0.3$ & $3.9 \pm 0.1$ & $4.8 \pm 0.1$ & $19 \pm 17$ & $1.11 \pm 0.99$ & 1 & \\
\hline & $0.521 \pm 0.004$ & $2.2 \pm 0.1$ & $10.8 \pm 0.1$ & $7.0 \pm 0.1$ & $2.5 \pm 0.1$ & $11.8 \pm 0.1$ & $19 \pm 3$ & $0.44 \pm 0.08$ & 3 & \\
\hline & $0.297 \pm 0.008$ & $1.1 \pm 0.1$ & $-2.2 \pm 0.1$ & $\ldots$ & $\ldots$ & $\ldots$ & $\ldots$ & $\ldots$ & $\ldots$ & \\
\hline & $0.975 \pm 0.015$ & $4.6 \pm 0.1$ & $-2.6 \pm 0.1$ & $\cdots$ & $\cdots$ & $\ldots$ & $\cdots$ & $\cdots$ & $\cdots$ & \\
\hline PKS0742 & $0.009 \pm 0.001$ & $3.2 \pm 0.1$ & $1.5 \pm 0.1$ & $1.5 \pm 0.1$ & $3.5 \pm 0.2$ & $1.6 \pm 0.1$ & $164 \pm 9$ & $0.09 \pm 0.01$ & 1 & 0.0 \\
\hline \multirow[t]{5}{*}{$3 \mathrm{C} 225 \mathrm{~A}$} & $0.043 \pm 0.002$ & $1.8 \pm 0.1$ & $-40.2 \pm 0.1$ & $0.9 \pm 0.0$ & $1.9 \pm 0.1$ & $-39.6 \pm 0.1$ & $22 \pm 0$ & $0.03 \pm 0.00$ & 5 & 1.0 \\
\hline & $0.020 \pm 0.002$ & $4.8 \pm 0.3$ & $-37.4 \pm 0.2$ & $1.2 \pm 0.0$ & $4.7 \pm 0.1$ & $-38.3 \pm 0.1$ & $60 \pm 1$ & $0.11 \pm 0.01$ & 5 & \\
\hline & $0.048 \pm 0.002$ & $2.5 \pm 0.1$ & $-27.2 \pm 0.1$ & $1.0 \pm 0.0$ & $2.8 \pm 0.1$ & $-27.7 \pm 0.1$ & $22 \pm 0$ & $0.05 \pm 0.00$ & 5 & \\
\hline & $0.013 \pm 0.002$ & $7.7 \pm 0.3$ & $-5.2 \pm 0.1$ & $4.3 \pm 0.1$ & $8.1 \pm 0.1$ & $-4.9 \pm 0.1$ & $327 \pm 11$ & $0.64 \pm 0.04$ & 5 & \\
\hline & $0.805 \pm 0.002$ & $1.3 \pm 0.1$ & $4.0 \pm 0.1$ & $5.7 \pm 0.0$ & $1.4 \pm 0.1$ & $3.6 \pm 0.1$ & $11 \pm 2$ & $0.23 \pm 0.04$ & 5 & \\
\hline \multirow[t]{5}{*}{$3 \mathrm{C} 225 \mathrm{~B}$} & $0.044 \pm 0.003$ & $2.0 \pm 0.1$ & $-40.3 \pm 0.1$ & $0.8 \pm 0.0$ & $1.9 \pm 0.1$ & $-39.8 \pm 0.1$ & $18 \pm 0$ & $0.03 \pm 0.00$ & 5 & 1.0 \\
\hline & $0.023 \pm 0.003$ & $4.0 \pm 0.3$ & $-37.2 \pm 0.1$ & $3.3 \pm 0.1$ & $4.0 \pm 0.1$ & $-37.4 \pm 0.1$ & $145 \pm 4$ & $0.26 \pm 0.02$ & 5 & \\
\hline & $0.053 \pm 0.003$ & $2.4 \pm 0.1$ & $-27.3 \pm 0.1$ & $1.0 \pm 0.0$ & $2.9 \pm 0.1$ & $-27.8 \pm 0.1$ & $20 \pm 0$ & $0.05 \pm 0.00$ & 5 & \\
\hline & $0.013 \pm 0.003$ & $8.3 \pm 0.4$ & $-5.6 \pm 0.2$ & $6.1 \pm 0.2$ & $8.3 \pm 0.1$ & $-4.5 \pm 0.1$ & $458 \pm 17$ & $1.00 \pm 0.07$ & 5 & \\
\hline & $0.774 \pm 0.003$ & $1.3 \pm 0.1$ & $4.0 \pm 0.1$ & $7.7 \pm 0.0$ & $1.5 \pm 0.1$ & $3.5 \pm 0.1$ & $14 \pm 0$ & $0.28 \pm 0.01$ & 5 & \\
\hline $3 \mathrm{C} 236$ & $\cdots$ & $\cdots$ & $\cdots$ & $\cdots$ & $\cdots$ & $\cdots$ & $\cdots$ & $\cdots$ & $\cdots$ & \\
\hline \multirow[t]{3}{*}{$3 \mathrm{C} 237$} & $0.006 \pm 0.001$ & $15.0 \pm 0.6$ & $-4.0 \pm 0.3$ & $2.1 \pm 0.1$ & $14.8 \pm 0.1$ & $-4.0 \pm 0.1$ & $382 \pm 17$ & $0.63 \pm 0.05$ & 3 & 1.0 \\
\hline & $0.415 \pm 0.001$ & $1.2 \pm 0.1$ & $2.3 \pm 0.1$ & $4.3 \pm 0.0$ & $1.2 \pm 0.1$ & $2.1 \pm 0.1$ & $13 \pm 0$ & $0.13 \pm 0.00$ & 3 & \\
\hline & $0.005 \pm 0.001$ & $1.9 \pm 0.2$ & $-2.4 \pm 0.1$ & $\ldots$ & $\ldots$ & $\ldots$ & $\ldots$ & $\ldots$ & $\cdots$ & \\
\hline \multirow[t]{2}{*}{$3 \mathrm{C} 245 \mathrm{~A}$} & $0.010 \pm 0.002$ & $5.3 \pm 0.3$ & $-9.1 \pm 0.1$ & $3.9 \pm 0.3$ & $5.3 \pm 0.1$ & $-8.8 \pm 0.1$ & $385 \pm 30$ & $0.40 \pm 0.05$ & 2 & 0.5 \\
\hline & $0.006 \pm 0.002$ & $1.6 \pm 0.3$ & $-9.8 \pm 0.1$ & $\ldots$ & $\ldots$ & $\ldots$ & $\ldots$ & $\ldots$ & $\cdots$ & \\
\hline $3 \mathrm{C} 245 \mathrm{~B}$ & $\cdots$ & $\cdots$ & $\cdots$ & $\cdots$ & $\cdots$ & $\cdots$ & $\cdots$ & $\cdots$ & $\cdots$ & \\
\hline $1055+018$ & $0.006 \pm 0.001$ & $7.1 \pm 0.3$ & $-7.3 \pm 0.1$ & $6.0 \pm 0.2$ & $7.0 \pm 0.1$ & $-6.8 \pm 0.1$ & $941 \pm 30$ & $0.83 \pm 0.05$ & 1 & 1.0 \\
\hline \multirow[t]{3}{*}{$3 \mathrm{C} 263.1$} & $0.007 \pm 0.001$ & $1.3 \pm 0.1$ & $-68.9 \pm 0.1$ & $0.1 \pm 0.0$ & $1.4 \pm 0.1$ & $-65.4 \pm 0.1$ & $15 \pm 1$ & $0.00 \pm 0.00$ & 3 & 1.0 \\
\hline & $0.020 \pm 0.001$ & $2.0 \pm 0.1$ & $-52.8 \pm 0.1$ & $0.7 \pm 0.0$ & $2.1 \pm 0.1$ & $-54.3 \pm 0.1$ & $35 \pm 0$ & $0.03 \pm 0.00$ & 3 & \\
\hline & $0.007 \pm 0.001$ & $0.6 \pm 0.1$ & $15.6 \pm 0.1$ & $0.3 \pm 0.0$ & $0.6 \pm 0.1$ & $16.9 \pm 0.1$ & $42 \pm 5$ & $0.00 \pm 0.00$ & 3 & \\
\hline \multirow[t]{3}{*}{$3 \mathrm{C} 273$} & $0.019 \pm 0.001$ & $2.3 \pm 0.1$ & $-6.3 \pm 0.1$ & $0.3 \pm 0.0$ & $2.5 \pm 0.1$ & $-5.7 \pm 0.1$ & $17 \pm 0$ & $0.02 \pm 0.00$ & 3 & 1.0 \\
\hline & $0.005 \pm 0.001$ & $6.4 \pm 0.3$ & $-5.8 \pm 0.1$ & $2.4 \pm 0.2$ & $7.0 \pm 0.1$ & $-5.7 \pm 0.1$ & $455 \pm 47$ & $0.29 \pm 0.04$ & 3 & \\
\hline & $0.003 \pm 0.001$ & $2.0 \pm 0.2$ & $31.6 \pm 0.1$ & $\ldots$ & $\ldots$ & $\ldots$ & $\ldots$ & $\ldots$ & $\cdots$ & \\
\hline \multirow[t]{2}{*}{$4 \mathrm{C} 32.44$} & $0.018 \pm 0.001$ & $2.8 \pm 0.1$ & $-16.2 \pm 0.1$ & $2.0 \pm 0.0$ & $3.1 \pm 0.1$ & $-16.5 \pm 0.1$ & $112 \pm 2$ & $0.11 \pm 0.00$ & 2 & 1.0 \\
\hline & $0.004 \pm 0.001$ & $3.7 \pm 0.3$ & $-4.2 \pm 0.1$ & $0.9 \pm 0.1$ & $3.9 \pm 0.1$ & $-4.6 \pm 0.1$ & $255 \pm 17$ & $0.07 \pm 0.01$ & 2 & \\
\hline
\end{tabular}

$4 \mathrm{C} 25.43$ 
Table 5

(Continued)

\begin{tabular}{|c|c|c|c|c|c|c|c|c|c|c|}
\hline $\begin{array}{l}\text { Source } \\
\text { (name) }\end{array}$ & $\tau_{0}$ & $\begin{array}{c}\Delta v_{0} \\
\left(\mathrm{~km} \mathrm{~s}^{-1}\right)\end{array}$ & $\begin{array}{c}v_{0} \\
\left(\mathrm{~km} \mathrm{~s}^{-1}\right)\end{array}$ & $\begin{array}{l}T_{B, n} \\
(\mathrm{~K})\end{array}$ & $\begin{array}{c}\Delta v_{0, n} \\
\left(\mathrm{~km} \mathrm{~s}^{-1}\right)\end{array}$ & $\begin{array}{c}v_{0, n} \\
\left(\mathrm{~km} \mathrm{~s}^{-1}\right)\end{array}$ & $\begin{array}{c}T_{s} \\
(\mathrm{~K})\end{array}$ & $\begin{array}{c}N(\mathrm{H} \mathrm{I})_{\mathrm{abs}} \\
\left(10^{20} \mathrm{~cm}^{-2}\right)\end{array}$ & (1) & $\mathscr{F}$ \\
\hline $3 C 286$ & $\begin{array}{l}0.006 \pm 0.001 \\
0.005 \pm 0.001 \\
0.007 \pm 0.001\end{array}$ & $\begin{array}{l}2.4 \pm 0.1 \\
3.2 \pm 0.2 \\
4.3 \pm 0.1\end{array}$ & $\begin{array}{r}-28.5 \pm 0.1 \\
-14.2 \pm 0.1 \\
-7.3 \pm 0.1\end{array}$ & $\begin{array}{l}0.5 \pm 0.0 \\
0.3 \pm 0.0 \\
0.6 \pm 0.0\end{array}$ & $\begin{array}{l}2.6 \pm 0.1 \\
3.5 \pm 0.1 \\
4.8 \pm 0.1\end{array}$ & $\begin{array}{r}-28.8 \pm 0.1 \\
-13.6 \pm 0.1 \\
-6.6 \pm 0.1\end{array}$ & $\begin{array}{l}76 \pm 2 \\
60 \pm 2 \\
78 \pm 2\end{array}$ & $\begin{array}{l}0.02 \pm 0.00 \\
0.02 \pm 0.00 \\
0.05 \pm 0.00\end{array}$ & $\begin{array}{l}3 \\
3 \\
3\end{array}$ & 0.0 \\
\hline $4 \mathrm{C} 12.50$ & $\begin{array}{l}0.016 \pm 0.001 \\
0.077 \pm 0.002\end{array}$ & $\begin{array}{l}6.6 \pm 0.1 \\
2.2 \pm 0.1\end{array}$ & $\begin{array}{l}-2.5 \pm 0.1 \\
-1.0 \pm 0.1\end{array}$ & $\begin{array}{l}5.1 \pm 0.2 \\
7.2 \pm 0.1\end{array}$ & $\begin{array}{l}6.5 \pm 0.1 \\
2.4 \pm 0.1\end{array}$ & $\begin{array}{l}-2.5 \pm 0.1 \\
-1.0 \pm 0.1\end{array}$ & $\begin{array}{c}318 \pm 11 \\
97 \pm 1\end{array}$ & $\begin{array}{l}0.66 \pm 0.04 \\
0.33 \pm 0.01\end{array}$ & $\begin{array}{l}2 \\
1\end{array}$ & 1.0 \\
\hline 3C298 & $0.019 \pm 0.001$ & $3.6 \pm 0.1$ & $-1.1 \pm 0.1$ & $2.1 \pm 0.2$ & $4.3 \pm 0.4$ & $-1.1 \pm 0.2$ & $112 \pm 10$ & $0.15 \pm 0.01$ & 1 & 0.5 \\
\hline UGC09799 & $0.058 \pm 0.010$ & $2.7 \pm 0.1$ & $-4.2 \pm 0.1$ & $3.1 \pm 0.2$ & $2.9 \pm 0.1$ & $-4.2 \pm 0.1$ & $58 \pm 4$ & $0.17 \pm 0.02$ & 1 & 1.0 \\
\hline $4 \mathrm{C} 04.51$ & $\begin{array}{l}0.002 \pm 0.001 \\
0.025 \pm 0.001 \\
0.067 \pm 0.001\end{array}$ & $\begin{array}{r}14.0 \pm 1.5 \\
2.4 \pm 0.1 \\
3.4 \pm 0.1\end{array}$ & $\begin{array}{l}-9.2 \pm 0.8 \\
-4.0 \pm 0.1 \\
-0.6 \pm 0.1\end{array}$ & $\begin{array}{r}0.3 \pm 0.0 \\
5.2 \pm 0.1 \\
12.1 \pm 0.1\end{array}$ & $\begin{array}{r}15.5 \pm 0.1 \\
2.4 \pm 0.1 \\
3.8 \pm 0.1\end{array}$ & $\begin{array}{l}-9.1 \pm 0.1 \\
-4.0 \pm 0.1 \\
-0.6 \pm 0.1\end{array}$ & $\begin{array}{l}114 \pm 8 \\
207 \pm 5 \\
183 \pm 2\end{array}$ & $\begin{array}{l}0.07 \pm 0.01 \\
0.24 \pm 0.01 \\
0.82 \pm 0.02\end{array}$ & $\begin{array}{l}3 \\
3 \\
3\end{array}$ & 0.0 \\
\hline $3 \mathrm{C} 327.1 \mathrm{~A}$ & $\begin{array}{l}0.126 \pm 0.006 \\
0.425 \pm 0.010 \\
0.401 \pm 0.008\end{array}$ & $\begin{array}{l}3.4 \pm 0.1 \\
1.9 \pm 0.1 \\
2.2 \pm 0.1\end{array}$ & $\begin{array}{r}-2.7 \pm 0.1 \\
-0.0 \pm 0.1 \\
2.0 \pm 0.1\end{array}$ & $\begin{array}{l}16.3 \pm 0.3 \\
19.8 \pm 0.6 \\
22.2 \pm 0.4\end{array}$ & $\begin{array}{l}3.7 \pm 0.1 \\
2.0 \pm 0.1 \\
2.4 \pm 0.1\end{array}$ & $\begin{array}{r}-2.9 \pm 0.1 \\
-0.0 \pm 0.1 \\
2.0 \pm 0.1\end{array}$ & $\begin{array}{r}140 \pm 3 \\
63 \pm 4 \\
69 \pm 3\end{array}$ & $\begin{array}{l}1.16 \pm 0.06 \\
1.00 \pm 0.08 \\
1.18 \pm 0.06\end{array}$ & $\begin{array}{l}1 \\
2 \\
3\end{array}$ & 1.0 \\
\hline $3 \mathrm{C} 327.1 \mathrm{~B}$ & $\begin{array}{l}0.118 \pm 0.006 \\
0.359 \pm 0.011 \\
0.419 \pm 0.008 \\
0.013 \pm 0.003\end{array}$ & $\begin{array}{l}3.2 \pm 0.1 \\
1.9 \pm 0.1 \\
2.2 \pm 0.1 \\
0.7 \pm 0.1\end{array}$ & $\begin{array}{r}-2.7 \pm 0.1 \\
-0.0 \pm 0.1 \\
1.9 \pm 0.1 \\
46.1 \pm 0.1\end{array}$ & $\begin{array}{c}15.6 \pm 0.3 \\
20.3 \pm 0.8 \\
22.2 \pm 0.6 \\
\ldots\end{array}$ & $\begin{array}{l}3.6 \pm 0.1 \\
2.1 \pm 0.1 \\
2.4 \pm 0.1 \\
\quad \cdots\end{array}$ & $\begin{array}{c}-3.0 \pm 0.1 \\
-0.0 \pm 0.1 \\
1.9 \pm 0.1 \\
\cdots\end{array}$ & $\begin{array}{r}142 \pm 3 \\
74 \pm 4 \\
68 \pm 3 \\
\cdots\end{array}$ & $\begin{array}{c}1.05 \pm 0.06 \\
1.01 \pm 0.08 \\
1.20 \pm 0.07 \\
\ldots\end{array}$ & $\begin{array}{r}4 \\
4 \\
4 \\
\cdots\end{array}$ & 1.0 \\
\hline PKS1607 & $\begin{array}{l}0.128 \pm 0.001 \\
0.013 \pm 0.001 \\
0.064 \pm 0.004 \\
0.123 \pm 0.004\end{array}$ & $\begin{array}{l}2.0 \pm 0.1 \\
6.5 \pm 0.3 \\
3.9 \pm 0.1 \\
2.2 \pm 0.1\end{array}$ & $\begin{array}{r}-10.3 \pm 0.1 \\
-7.8 \pm 0.2 \\
-1.2 \pm 0.1 \\
-2.3 \pm 0.1\end{array}$ & $\begin{array}{c}3.0 \pm 0.0 \\
1.4 \pm 0.0 \\
7.0 \pm 0.4 \\
\ldots\end{array}$ & $\begin{aligned} 2.0 & \pm 0.1 \\
7.2 & \pm 0.1 \\
4.3 & \pm 0.1 \\
\ldots & \end{aligned}$ & $\begin{array}{c}-10.7 \pm 0.1 \\
-7.8 \pm 0.1 \\
-1.1 \pm 0.1 \\
\cdots\end{array}$ & $\begin{array}{c}26 \pm 1 \\
114 \pm 10 \\
115 \pm 10 \\
\ldots\end{array}$ & $\begin{array}{c}0.13 \pm 0.01 \\
0.19 \pm 0.02 \\
0.56 \pm 0.06 \\
\ldots\end{array}$ & $\begin{array}{r}1 \\
2 \\
4 \\
\cdots\end{array}$ & 1.0 \\
\hline $\mathrm{J} 1613$ & $\cdots$ & $\cdots$ & $\cdots$ & $\cdots$ & $\cdots$ & $\cdots$ & $\cdots$ & $\cdots$ & $\cdots$ & \\
\hline $3 \mathrm{C} 345$ & $0.009 \pm 0.001$ & $0.6 \pm 0.1$ & $-5.2 \pm 0.1$ & $\cdots$ & $\ldots$ & $\cdots$ & $\cdots$ & $\cdots$ & $\cdots$ & 0.0 \\
\hline $3 \mathrm{C} 346$ & $\begin{array}{l}0.035 \pm 0.003 \\
0.279 \pm 0.004 \\
0.197 \pm 0.004\end{array}$ & $\begin{array}{l}4.5 \pm 0.1 \\
2.0 \pm 0.1 \\
1.9 \pm 0.1\end{array}$ & $\begin{array}{r}-6.4 \pm 0.1 \\
-0.6 \pm 0.1 \\
2.1 \pm 0.1\end{array}$ & $\begin{array}{r}4.5 \pm 0.1 \\
13.2 \pm 0.1 \\
9.8 \pm 0.1\end{array}$ & $\begin{array}{l}4.9 \pm 0.1 \\
2.1 \pm 0.1 \\
2.1 \pm 0.1\end{array}$ & $\begin{array}{r}-6.4 \pm 0.1 \\
-0.6 \pm 0.1 \\
2.1 \pm 0.1\end{array}$ & $\begin{array}{r}133 \pm 3 \\
58 \pm 4 \\
59 \pm 4\end{array}$ & $\begin{array}{l}0.40 \pm 0.02 \\
0.62 \pm 0.04 \\
0.43 \pm 0.03\end{array}$ & $\begin{array}{l}3 \\
3 \\
3\end{array}$ & 1.0 \\
\hline $3 C 390$ & $\begin{array}{l}0.103 \pm 0.006 \\
0.146 \pm 0.003 \\
0.082 \pm 0.003 \\
0.043 \pm 0.003 \\
0.095 \pm 0.003 \\
0.067 \pm 0.003 \\
0.007 \pm 0.002 \\
0.022 \pm 0.002 \\
0.033 \pm 0.003 \\
0.006 \pm 0.002\end{array}$ & $\begin{array}{l}2.2 \pm 0.1 \\
3.7 \pm 0.2 \\
5.7 \pm 0.2 \\
2.0 \pm 0.1 \\
9.8 \pm 0.1 \\
1.4 \pm 0.1 \\
9.2 \pm 1.1 \\
1.7 \pm 0.1 \\
2.0 \pm 0.1 \\
0.6 \pm 0.2\end{array}$ & $\begin{array}{r}1.0 \pm 0.1 \\
3.7 \pm 0.1 \\
8.8 \pm 0.2 \\
11.2 \pm 0.1 \\
24.5 \pm 0.1 \\
28.7 \pm 0.1 \\
42.2 \pm 0.3 \\
35.8 \pm 0.1 \\
24.0 \pm 0.1 \\
17.1 \pm 0.1\end{array}$ & $\begin{aligned} & 14.6 \pm 0.8 \\
& 30.5 \pm 0.6 \\
& 26.2 \pm 0.4 \\
& 1.1 \pm 0.1 \\
& 37.7 \pm 0.5 \\
& 2.0 \pm 0.0 \\
& 18.1 \pm 1.1 \\
& \ldots \ldots \\
& \cdots\end{aligned}$ & $\begin{aligned} 2.5 & \pm 0.1 \\
4.0 & \pm 0.1 \\
6.3 & \pm 0.1 \\
2.2 & \pm 0.1 \\
10.7 & \pm 0.1 \\
1.5 & \pm 0.1 \\
10.2 & \pm 0.1 \\
\ldots & \\
\ldots & \end{aligned}$ & $\begin{aligned} 0.9 & \pm 0.1 \\
3.4 & \pm 0.1 \\
8.8 & \pm 0.1 \\
12.3 & \pm 0.1 \\
25.5 & \pm 0.1 \\
30.8 & \pm 0.1 \\
42.1 & \pm 0.1 \\
\ldots & \\
\ldots & \end{aligned}$ & $\begin{array}{c}143 \pm 11 \\
221 \pm 7 \\
331 \pm 7 \\
27 \pm 4 \\
415 \pm 6 \\
30 \pm 4 \\
2613 \pm 177 \\
\ldots \\
\ldots \\
\ldots\end{array}$ & $\begin{array}{c}0.64 \pm 0.06 \\
2.31 \pm 0.14 \\
3.01 \pm 0.13 \\
0.05 \pm 0.01 \\
7.51 \pm 0.17 \\
0.06 \pm 0.01 \\
3.24 \pm 0.48 \\
\ldots \\
\ldots \\
\ldots\end{array}$ & $\begin{array}{r}10 \\
3 \\
10 \\
10 \\
1 \\
10 \\
2 \\
\cdots \\
\cdots \\
\ldots\end{array}$ & 0.5 \\
\hline $4 C 33.48$ & $\begin{array}{l}0.170 \pm 0.006 \\
0.139 \pm 0.006 \\
0.277 \pm 0.006 \\
0.128 \pm 0.005\end{array}$ & $\begin{array}{l}2.2 \pm 0.1 \\
6.8 \pm 0.1 \\
2.3 \pm 0.1 \\
1.7 \pm 0.1\end{array}$ & $\begin{array}{r}3.5 \pm 0.1 \\
25.4 \pm 0.1 \\
23.8 \pm 0.1 \\
28.8 \pm 0.1\end{array}$ & $\begin{array}{c}3.6 \pm 0.1 \\
8.5 \pm 0.3 \\
\ldots \\
\ldots\end{array}$ & $\begin{array}{c}2.4 \pm 0.1 \\
6.8 \pm 0.1 \\
\ldots \\
\ldots\end{array}$ & $\begin{array}{c}3.3 \pm 0.1 \\
23.5 \pm 0.1 \\
\ldots \\
\ldots\end{array}$ & $\begin{array}{c}26 \pm 5 \\
75 \pm 13 \\
\ldots \\
\ldots\end{array}$ & $\begin{array}{c}0.20 \pm 0.04 \\
1.39 \pm 0.25 \\
\ldots \\
\ldots\end{array}$ & $\begin{array}{r}4 \\
4 \\
\cdots \\
\cdots\end{array}$ & 1.0 \\
\hline $3 \mathrm{C} 409 \mathrm{~A}$ & $\begin{array}{l}0.443 \pm 0.004 \\
0.332 \pm 0.005 \\
0.732 \pm 0.007 \\
0.440 \pm 0.008 \\
0.735 \pm 0.006 \\
0.020 \pm 0.003 \\
0.004 \pm 0.002\end{array}$ & $\begin{array}{l}3.2 \pm 0.1 \\
3.0 \pm 0.1 \\
2.1 \pm 0.1 \\
6.5 \pm 0.1 \\
1.7 \pm 0.1 \\
4.3 \pm 0.2 \\
0.6 \pm 0.2\end{array}$ & $\begin{array}{r}4.2 \pm 0.1 \\
7.9 \pm 0.1 \\
13.8 \pm 0.1 \\
14.6 \pm 0.1 \\
15.9 \pm 0.1 \\
24.3 \pm 0.1 \\
-53.8 \pm 0.1\end{array}$ & $\begin{aligned} 23.6 & \pm 0.1 \\
34.0 & \pm 0.2 \\
5.8 & \pm 0.1 \\
49.2 & \pm 1.2 \\
0.6 & \pm 0.1 \\
7.1 & \pm 0.3 \\
& \ldots\end{aligned}$ & $\begin{array}{l}3.5 \pm 0.1 \\
3.0 \pm 0.1 \\
2.1 \pm 0.1 \\
6.8 \pm 0.1 \\
1.6 \pm 0.1 \\
4.8 \pm 0.1 \\
\ldots\end{array}$ & $\begin{aligned} 4.2 & \pm 0.1 \\
7.9 & \pm 0.1 \\
12.5 & \pm 0.1 \\
14.5 & \pm 0.1 \\
15.9 & \pm 0.1 \\
24.6 & \pm 0.1 \\
\ldots & \end{aligned}$ & $\begin{aligned} & 64 \pm 9 \\
& 122 \pm 11 \\
& 13 \pm 5 \\
& 145 \pm 20 \\
& 12 \pm 18 \\
& 366 \pm 39 \\
& \cdots\end{aligned}$ & $\begin{array}{c}1.77 \pm 0.27 \\
2.36 \pm 0.22 \\
0.41 \pm 0.16 \\
8.11 \pm 1.18 \\
0.29 \pm 0.45 \\
0.63 \pm 0.08 \\
\ldots\end{array}$ & $\begin{array}{r}1 \\
2 \\
7 \\
7 \\
7 \\
7 \\
\cdots\end{array}$ & 0.5 \\
\hline $3 \mathrm{C} 409 \mathrm{~B}$ & $\begin{array}{l}0.429 \pm 0.003 \\
0.280 \pm 0.007 \\
0.631 \pm 0.081 \\
0.106 \pm 0.004 \\
0.890 \pm 0.065 \\
0.193 \pm 0.053\end{array}$ & $\begin{aligned} 2.9 & \pm 0.1 \\
3.0 & \pm 0.1 \\
1.9 & \pm 0.1 \\
13.1 & \pm 0.2 \\
3.0 & \pm 0.1 \\
3.6 & \pm 1.2\end{aligned}$ & $\begin{array}{r}4.0 \pm 0.1 \\
8.0 \pm 0.1 \\
13.6 \pm 0.1 \\
14.5 \pm 0.2 \\
15.3 \pm 0.1 \\
12.3 \pm 0.9\end{array}$ & $\begin{aligned} 14.0 & \pm 0.1 \\
24.9 & \pm 0.8 \\
7.2 & \pm 5.2 \\
28.5 & \pm 1.1 \\
16.7 & \pm 3.0 \\
& \ldots\end{aligned}$ & $\begin{aligned} & 3.2 \pm 0.1 \\
& 3.1 \pm 0.1 \\
& 1.8 \pm 0.1 \\
& 14.4 \pm 0.1 \\
& 3.0 \pm 0.1 \\
& \ldots\end{aligned}$ & $\begin{array}{r}3.6 \pm 0.1 \\
8.0 \pm 0.1 \\
13.9 \pm 0.1 \\
15.9 \pm 0.1 \\
15.6 \pm 0.1 \\
\ldots\end{array}$ & $\begin{aligned} & 49 \pm 9 \\
& 120 \pm 13 \\
& 66 \pm 22 \\
& 285 \pm 12 \\
& 47 \pm 13 \\
& \ldots\end{aligned}$ & $\begin{array}{c}1.18 \pm 0.24 \\
1.97 \pm 0.23 \\
1.51 \pm 0.55 \\
7.67 \pm 0.46 \\
2.45 \pm 0.73 \\
\ldots\end{array}$ & $\begin{array}{r}1 \\
3 \\
6 \\
6 \\
2 \\
\cdots\end{array}$ & 1.0 \\
\hline
\end{tabular}


Table 5

(Continued)

\begin{tabular}{|c|c|c|c|c|c|c|c|c|c|c|}
\hline $\begin{array}{l}\text { Source } \\
\text { (name) }\end{array}$ & $\tau_{0}$ & $\begin{array}{c}\Delta v_{0} \\
\left(\mathrm{~km} \mathrm{~s}^{-1}\right)\end{array}$ & $\begin{array}{c}v_{0} \\
\left(\mathrm{~km} \mathrm{~s}^{-1}\right)\end{array}$ & $\begin{array}{l}T_{B, n} \\
(\mathrm{~K})\end{array}$ & $\underset{\left(\mathrm{km} \mathrm{s}^{-1}\right)}{\Delta v_{0, n}}$ & $\begin{array}{c}v_{0, n} \\
\left(\mathrm{~km} \mathrm{~s}^{-1}\right)\end{array}$ & $\begin{array}{c}T_{s} \\
(\mathrm{~K})\end{array}$ & $\begin{array}{c}N(\mathrm{H} \mathrm{I})_{\mathrm{abs}} \\
\left(10^{20} \mathrm{~cm}^{-2}\right)\end{array}$ & (1) & $\mathscr{F}$ \\
\hline \multirow[t]{13}{*}{$3 \mathrm{C} 410 \mathrm{~A}$} & $0.014 \pm 0.002$ & $1.5 \pm 0.1$ & $-30.2 \pm 0.1$ & $0.2 \pm 0.0$ & $1.5 \pm 0.1$ & $-30.2 \pm 0.1$ & $15 \pm 3$ & $0.01 \pm 0.00$ & 13 & 1.0 \\
\hline & $0.020 \pm 0.003$ & $4.0 \pm 0.2$ & $-22.7 \pm 0.1$ & $12.6 \pm 0.6$ & $4.0 \pm 0.1$ & $-23.4 \pm 0.1$ & $647 \pm 31$ & $0.99 \pm 0.09$ & 13 & \\
\hline & $0.613 \pm 0.018$ & $3.6 \pm 0.1$ & $-0.2 \pm 0.1$ & $17.5 \pm 0.9$ & $3.9 \pm 0.1$ & $-0.2 \pm 0.1$ & $48 \pm 12$ & $2.07 \pm 0.55$ & 13 & \\
\hline & $0.648 \pm 0.023$ & $3.2 \pm 0.1$ & $2.7 \pm 0.1$ & $17.9 \pm 1.1$ & $3.6 \pm 0.1$ & $2.7 \pm 0.1$ & $49 \pm 14$ & $2.02 \pm 0.60$ & 13 & \\
\hline & $1.693 \pm 0.150$ & $3.2 \pm 0.1$ & $7.4 \pm 0.1$ & $47.0 \pm 9.9$ & $3.5 \pm 0.1$ & $6.7 \pm 0.1$ & $70 \pm 13$ & $7.33 \pm 1.64$ & 13 & \\
\hline & $1.864 \pm 0.125$ & $1.5 \pm 0.1$ & $8.1 \pm 0.1$ & $11.6 \pm 12.2$ & $1.6 \pm 0.1$ & $8.1 \pm 0.1$ & $104 \pm 60$ & $5.79 \pm 3.46$ & 13 & \\
\hline & $0.149 \pm 0.081$ & $1.6 \pm 0.3$ & $11.1 \pm 0.1$ & $8.5 \pm 8.3$ & $1.8 \pm 0.1$ & $10.6 \pm 0.1$ & $106 \pm 45$ & $0.50 \pm 0.37$ & 13 & \\
\hline & $0.575 \pm 0.096$ & $4.4 \pm 0.4$ & $11.3 \pm 0.4$ & $43.6 \pm 10.2$ & $4.4 \pm 0.1$ & $11.6 \pm 0.1$ & $111 \pm 21$ & $5.46 \pm 1.48$ & 13 & \\
\hline & $0.186 \pm 0.003$ & $3.3 \pm 0.1$ & $17.9 \pm 0.1$ & $10.9 \pm 0.1$ & $3.3 \pm 0.1$ & $16.5 \pm 0.1$ & $71 \pm 8$ & $0.86 \pm 0.11$ & 13 & \\
\hline & $0.060 \pm 0.003$ & $5.2 \pm 0.1$ & $25.3 \pm 0.1$ & $3.5 \pm 0.2$ & $5.8 \pm 0.1$ & $25.3 \pm 0.1$ & $69 \pm 14$ & $0.43 \pm 0.09$ & 13 & \\
\hline & $0.055 \pm 0.002$ & $1.9 \pm 0.1$ & $-46.5 \pm 0.1$ & $\ldots$ & $\ldots$ & $\ldots$ & $\ldots$ & $\ldots$ & $\cdots$ & \\
\hline & $0.048 \pm 0.003$ & $3.0 \pm 0.2$ & $-4.8 \pm 0.1$ & $\cdots$ & $\cdots$ & $\cdots$ & $\cdots$ & $\cdots$ & $\cdots$ & \\
\hline & $0.076 \pm 0.003$ & $1.8 \pm 0.1$ & $24.6 \pm 0.1$ & $\cdots$ & $\cdots$ & $\cdots$ & $\cdots$ & $\cdots$ & $\cdots$ & \\
\hline \multirow[t]{12}{*}{$3 \mathrm{C} 410 \mathrm{~B}$} & $0.021 \pm 0.002$ & $5.3 \pm 0.4$ & $-47.6 \pm 0.2$ & $0.8 \pm 0.1$ & $5.8 \pm 0.1$ & $-52.0 \pm 0.1$ & $39 \pm 3$ & $0.08 \pm 0.01$ & 12 & 0.0 \\
\hline & $0.019 \pm 0.002$ & $1.8 \pm 0.3$ & $-30.2 \pm 0.1$ & $0.4 \pm 0.0$ & $2.0 \pm 0.1$ & $-30.1 \pm 0.1$ & $18 \pm 3$ & $0.01 \pm 0.00$ & 12 & \\
\hline & $0.476 \pm 0.007$ & $2.7 \pm 0.1$ & $-0.2 \pm 0.1$ & $12.2 \pm 0.1$ & $2.9 \pm 0.1$ & $-0.2 \pm 0.1$ & $28 \pm 2$ & $0.71 \pm 0.07$ & 12 & \\
\hline & $0.344 \pm 0.023$ & $11.4 \pm 0.5$ & $5.1 \pm 0.3$ & $46.3 \pm 3.5$ & $12.6 \pm 0.1$ & $4.6 \pm 0.1$ & $153 \pm 12$ & $11.71 \pm 1.36$ & 1 & \\
\hline & $0.292 \pm 0.011$ & $1.5 \pm 0.1$ & $5.9 \pm 0.1$ & $7.5 \pm 0.3$ & $1.4 \pm 0.1$ & $6.0 \pm 0.1$ & $31 \pm 3$ & $0.26 \pm 0.03$ & 12 & \\
\hline & $2.798 \pm 0.014$ & $2.2 \pm 0.1$ & $8.0 \pm 0.1$ & $17.2 \pm 0.2$ & $2.4 \pm 0.1$ & $8.0 \pm 0.1$ & $18 \pm 1$ & $2.15 \pm 0.24$ & 12 & \\
\hline & $0.430 \pm 0.137$ & $1.9 \pm 0.1$ & $10.9 \pm 0.1$ & $22.0 \pm 8.4$ & $2.0 \pm 0.1$ & $10.2 \pm 0.1$ & $65 \pm 21$ & $1.06 \pm 0.49$ & 12 & \\
\hline & $0.041 \pm 0.017$ & $19.2 \pm 2.0$ & $12.7 \pm 3.1$ & $40.7 \pm 16.7$ & $19.1 \pm 0.1$ & $11.4 \pm 0.1$ & $1006 \pm 403$ & $15.52 \pm 9.16$ & 12 & \\
\hline & $0.112 \pm 0.004$ & $2.5 \pm 0.1$ & $25.2 \pm 0.1$ & $3.9 \pm 0.1$ & $2.8 \pm 0.1$ & $25.1 \pm 0.1$ & $32 \pm 3$ & $0.18 \pm 0.02$ & 12 & \\
\hline & $0.426 \pm 0.010$ & $2.3 \pm 0.1$ & $2.5 \pm 0.1$ & $\ldots$ & $\ldots$ & $\ldots$ & $\ldots$ & $\ldots$ & $\cdots$ & \\
\hline & $0.119 \pm 0.005$ & $2.5 \pm 0.1$ & $18.4 \pm 0.1$ & $\cdots$ & $\cdots$ & $\cdots$ & $\cdots$ & $\ldots$ & .. & \\
\hline & $0.347 \pm 0.087$ & $2.6 \pm 0.3$ & $12.2 \pm 0.4$ & $\cdots$ & $\cdots$ & $\cdots$ & $\cdots$ & $\cdots$ & $\cdots$ & \\
\hline \multirow[t]{9}{*}{ B2050 } & $0.007 \pm 0.001$ & $3.4 \pm 0.2$ & $-21.3 \pm 0.1$ & $1.0 \pm 0.1$ & $3.7 \pm 0.1$ & $-21.2 \pm 0.1$ & $141 \pm 9$ & $0.06 \pm 0.01$ & 9 & 1.0 \\
\hline & $0.011 \pm 0.001$ & $2.4 \pm 0.1$ & $-11.5 \pm 0.1$ & $0.8 \pm 0.0$ & $2.7 \pm 0.1$ & $-12.5 \pm 0.1$ & $78 \pm 12$ & $0.04 \pm 0.01$ & 9 & \\
\hline & $0.043 \pm 0.002$ & $10.0 \pm 0.3$ & $-0.1 \pm 0.2$ & $18.2 \pm 1.0$ & $10.0 \pm 0.1$ & $-0.1 \pm 0.1$ & $453 \pm 29$ & $3.79 \pm 0.34$ & 9 & \\
\hline & $0.039 \pm 0.002$ & $2.0 \pm 0.1$ & $2.5 \pm 0.1$ & $0.6 \pm 0.0$ & $2.0 \pm 0.1$ & $2.7 \pm 0.1$ & $13 \pm 5$ & $0.02 \pm 0.01$ & 9 & \\
\hline & $0.150 \pm 0.002$ & $1.7 \pm 0.1$ & $9.0 \pm 0.1$ & $0.4 \pm 0.0$ & $1.7 \pm 0.1$ & $8.2 \pm 0.1$ & $10 \pm 6$ & $0.05 \pm 0.03$ & 2 & \\
\hline & $0.190 \pm 0.002$ & $6.0 \pm 0.1$ & $10.2 \pm 0.1$ & $21.5 \pm 0.3$ & $6.0 \pm 0.1$ & $9.4 \pm 0.1$ & $142 \pm 15$ & $3.16 \pm 0.35$ & 1 & \\
\hline & $0.074 \pm 0.002$ & $3.8 \pm 0.1$ & $15.9 \pm 0.1$ & $1.7 \pm 0.0$ & $4.1 \pm 0.1$ & $16.2 \pm 0.1$ & $28 \pm 6$ & $0.15 \pm 0.04$ & 9 & \\
\hline & $0.086 \pm 0.002$ & $2.8 \pm 0.1$ & $-0.8 \pm 0.1$ & $\ldots$ & $\ldots$ & $\ldots$ & $\ldots$ & $\ldots$ & $\ldots$ & \\
\hline & $0.014 \pm 0.002$ & $1.2 \pm 0.1$ & $-6.1 \pm 0.1$ & $\cdots$ & $\cdots$ & $\cdots$ & $\cdots$ & $\cdots$ & $\cdots$ & \\
\hline \multirow[t]{4}{*}{$3 C 433$} & $0.181 \pm 0.010$ & $1.5 \pm 0.1$ & $2.6 \pm 0.1$ & $2.2 \pm 0.2$ & $1.6 \pm 0.1$ & $2.4 \pm 0.1$ & $21 \pm 9$ & $0.11 \pm 0.05$ & 2 & 1.0 \\
\hline & $0.304 \pm 0.009$ & $3.9 \pm 0.1$ & $3.5 \pm 0.1$ & $19.1 \pm 0.7$ & $4.1 \pm 0.1$ & $3.5 \pm 0.1$ & $78 \pm 5$ & $1.82 \pm 0.15$ & 1 & \\
\hline & $0.080 \pm 0.005$ & $2.1 \pm 0.1$ & $7.3 \pm 0.1$ & $1.8 \pm 0.1$ & $2.1 \pm 0.1$ & $7.3 \pm 0.1$ & $29 \pm 5$ & $0.09 \pm 0.02$ & 4 & \\
\hline & $0.059 \pm 0.004$ & $2.5 \pm 0.1$ & $16.5 \pm 0.1$ & $1.6 \pm 0.0$ & $2.7 \pm 0.1$ & $15.7 \pm 0.1$ & $29 \pm 2$ & $0.08 \pm 0.01$ & 4 & \\
\hline \multirow[t]{3}{*}{ PKS2127 } & $0.073 \pm 0.001$ & $2.7 \pm 0.1$ & $-0.9 \pm 0.1$ & $7.6 \pm 0.1$ & $2.7 \pm 0.1$ & $-0.9 \pm 0.1$ & $105 \pm 4$ & $0.40 \pm 0.02$ & 1 & 1.0 \\
\hline & $0.013 \pm 0.001$ & $8.3 \pm 0.2$ & $1.1 \pm 0.1$ & $9.7 \pm 0.6$ & $8.3 \pm 0.1$ & $1.2 \pm 0.1$ & $769 \pm 43$ & $1.59 \pm 0.14$ & 2 & \\
\hline & $0.107 \pm 0.001$ & $2.1 \pm 0.1$ & $1.5 \pm 0.1$ & $5.8 \pm 0.1$ & $2.0 \pm 0.1$ & $1.5 \pm 0.1$ & $61 \pm 5$ & $0.27 \pm 0.02$ & 3 & \\
\hline \multirow[t]{3}{*}{$\mathrm{J} 2136$} & $0.096 \pm 0.002$ & $2.2 \pm 0.1$ & $1.1 \pm 0.1$ & $3.1 \pm 0.0$ & $2.4 \pm 0.1$ & $1.0 \pm 0.1$ & $28 \pm 3$ & $0.12 \pm 0.02$ & 1 & 0.0 \\
\hline & $0.062 \pm 0.002$ & $7.0 \pm 0.1$ & $3.4 \pm 0.1$ & $2.4 \pm 0.0$ & $7.7 \pm 0.1$ & $3.1 \pm 0.1$ & $22 \pm 13$ & $0.19 \pm 0.12$ & 2 & \\
\hline & $0.079 \pm 0.002$ & $2.5 \pm 0.1$ & $5.8 \pm 0.1$ & $7.7 \pm 0.1$ & $2.7 \pm 0.1$ & $6.3 \pm 0.1$ & $98 \pm 4$ & $0.37 \pm 0.02$ & 3 & \\
\hline \multirow[t]{5}{*}{$\mathrm{J} 2232$} & $0.066 \pm 0.001$ & $2.3 \pm 0.1$ & $-14.3 \pm 0.1$ & $4.2 \pm 0.0$ & $2.5 \pm 0.1$ & $-15.0 \pm 0.1$ & $65 \pm 0$ & $0.19 \pm 0.00$ & 5 & 0.5 \\
\hline & $0.056 \pm 0.002$ & $2.0 \pm 0.1$ & $-7.6 \pm 0.1$ & $3.1 \pm 0.1$ & $2.0 \pm 0.1$ & $-7.6 \pm 0.1$ & $58 \pm 3$ & $0.13 \pm 0.01$ & 5 & \\
\hline & $0.064 \pm 0.002$ & $5.7 \pm 0.1$ & $-5.0 \pm 0.1$ & $6.6 \pm 0.2$ & $5.7 \pm 0.1$ & $-5.0 \pm 0.1$ & $105 \pm 4$ & $0.75 \pm 0.04$ & 5 & \\
\hline & $0.099 \pm 0.002$ & $2.0 \pm 0.1$ & $-3.6 \pm 0.1$ & $2.9 \pm 0.1$ & $2.1 \pm 0.1$ & $-3.6 \pm 0.1$ & $32 \pm 4$ & $0.12 \pm 0.02$ & 5 & \\
\hline & $0.046 \pm 0.002$ & $3.3 \pm 0.1$ & $2.1 \pm 0.1$ & $4.3 \pm 0.1$ & $3.6 \pm 0.1$ & $2.3 \pm 0.1$ & $96 \pm 3$ & $0.28 \pm 0.01$ & 1 & \\
\hline \multirow[t]{8}{*}{$3 \mathrm{C} 454.3$} & $0.313 \pm 0.024$ & $2.5 \pm 0.1$ & $-10.3 \pm 0.1$ & $5.0 \pm 0.5$ & $2.8 \pm 0.1$ & $-10.6 \pm 0.1$ & $20 \pm 2$ & $0.31 \pm 0.04$ & 1 & 1.0 \\
\hline & $0.025 \pm 0.013$ & $3.7 \pm 1.2$ & $-8.5 \pm 1.3$ & $4.0 \pm 2.1$ & $3.7 \pm 0.1$ & $-8.1 \pm 0.1$ & $159 \pm 90$ & $0.28 \pm 0.24$ & 8 & \\
\hline & $0.096 \pm 0.002$ & $3.6 \pm 0.1$ & $-2.1 \pm 0.1$ & $12.9 \pm 0.1$ & $3.6 \pm 0.1$ & $-2.1 \pm 0.1$ & $142 \pm 2$ & $0.95 \pm 0.02$ & 8 & \\
\hline & $0.081 \pm 0.002$ & $1.8 \pm 0.1$ & $0.7 \pm 0.1$ & $4.6 \pm 0.1$ & $1.9 \pm 0.1$ & $0.6 \pm 0.1$ & $61 \pm 3$ & $0.17 \pm 0.01$ & 8 & \\
\hline & $0.026 \pm 0.002$ & $4.7 \pm 0.3$ & $3.0 \pm 0.2$ & $2.7 \pm 0.1$ & $5.1 \pm 0.1$ & $3.3 \pm 0.1$ & $109 \pm 3$ & $0.25 \pm 0.02$ & 8 & \\
\hline & $0.047 \pm 0.002$ & $2.1 \pm 0.1$ & $-30.5 \pm 0.1$ & $\ldots$ & $\ldots$ & $\ldots$ & $\ldots$ & $\ldots$ & $\ldots$ & \\
\hline & $0.016 \pm 0.002$ & $5.9 \pm 0.2$ & $-16.4 \pm 0.1$ & $\cdots$ & $\cdots$ & $\cdots$ & $\cdots$ & $\cdots$ & $\cdots$ & \\
\hline & $0.010 \pm 0.002$ & $3.5 \pm 0.2$ & $-35.1 \pm 0.1$ & $\cdots$ & $\cdots$ & $\cdots$ & $\cdots$ & $\cdots$ & $\cdots$ & \\
\hline
\end{tabular}


Table 5

(Continued)

\begin{tabular}{|c|c|c|c|c|c|c|c|c|c|c|}
\hline $\begin{array}{l}\text { Source } \\
\text { (name) }\end{array}$ & $\tau_{0}$ & $\begin{array}{c}\Delta v_{0} \\
\left(\mathrm{~km} \mathrm{~s}^{-1}\right)\end{array}$ & $\begin{array}{c}v_{0} \\
\left(\mathrm{~km} \mathrm{~s}^{-1}\right)\end{array}$ & $\begin{array}{l}T_{B, n} \\
(\mathrm{~K})\end{array}$ & $\begin{array}{c}\Delta v_{0, n} \\
\left(\mathrm{~km} \mathrm{~s}^{-1}\right)\end{array}$ & $\begin{array}{c}v_{0, n} \\
\left(\mathrm{~km} \mathrm{~s}^{-1}\right)\end{array}$ & $\begin{array}{c}T_{s} \\
(\mathrm{~K})\end{array}$ & $\begin{array}{c}N(\mathrm{H} \mathrm{I})_{\mathrm{abs}} \\
\left(10^{20} \mathrm{~cm}^{-2}\right)\end{array}$ & (1) & $\mathscr{F}$ \\
\hline \multirow[t]{7}{*}{$3 \mathrm{C} 459$} & $0.010 \pm 0.001$ & $2.8 \pm 0.1$ & $-13.2 \pm 0.1$ & $0.7 \pm 0.0$ & $3.1 \pm 0.1$ & $-12.4 \pm 0.1$ & $72 \pm 2$ & $0.04 \pm 0.00$ & 2 & 1.0 \\
\hline & $0.039 \pm 0.002$ & $5.2 \pm 0.3$ & $-6.2 \pm 0.2$ & $14.4 \pm 0.8$ & $5.5 \pm 0.1$ & $-6.7 \pm 0.1$ & $384 \pm 22$ & $1.48 \pm 0.14$ & 1 & \\
\hline & $0.057 \pm 0.002$ & $1.9 \pm 0.1$ & $0.4 \pm 0.1$ & $2.7 \pm 0.1$ & $2.0 \pm 0.1$ & $0.4 \pm 0.1$ & $37 \pm 8$ & $0.08 \pm 0.02$ & 7 & \\
\hline & $0.039 \pm 0.003$ & $7.7 \pm 0.6$ & $0.8 \pm 0.3$ & $18.3 \pm 1.4$ & $7.7 \pm 0.1$ & $0.7 \pm 0.1$ & $478 \pm 35$ & $2.79 \pm 0.37$ & 7 & \\
\hline & $0.088 \pm 0.003$ & $2.2 \pm 0.1$ & $2.9 \pm 0.1$ & $3.5 \pm 0.1$ & $2.2 \pm 0.1$ & $2.9 \pm 0.1$ & $46 \pm 6$ & $0.18 \pm 0.03$ & 7 & \\
\hline & $0.016 \pm 0.001$ & $1.4 \pm 0.1$ & $7.7 \pm 0.1$ & $0.9 \pm 0.0$ & $1.6 \pm 0.1$ & $7.7 \pm 0.1$ & $56 \pm 2$ & $0.03 \pm 0.00$ & 7 & \\
\hline & $0.102 \pm 0.003$ & $2.2 \pm 0.1$ & $-7.3 \pm 0.1$ & $\ldots$ & $\ldots$ & $\ldots$ & $\ldots$ & $\ldots$ & $\ldots$ & \\
\hline
\end{tabular}

Note. Cols. (2)-(4): Gaussian parameters fit to H I absorption (Equation (1)). Cols. (5)-(7): Gaussian parameters fit to H I emission (Equations (2)). Col. (8): average spin temperature from all permutations of components with overlap along the LOS (Equations (3), (4)). Col. (9): column density computed from fitted parameters (Equation (6)). Col. (10): order of components along the LOS corresponding to the smallest model residuals. Components whose position along the LOS is extremely uncertain or unaffected by order permutations are assumed to lie behind all others (i.e., $\mathcal{O}=N$, for $N$ total components). Col (11): fraction of WNM (emissiondetected only) components that lie in front of all absorption-detected components, allowed to be 1.0 or 0.0 for all emission-detected components. Fit parameters for components with $T_{s} \leqslant 3 \mathrm{~K}$ are omitted, as these are either spurious AGD detections or were not recovered in the fit to $T_{B, \exp }(v)$ due to strong line blending.

\section{ORCID iDs}

Claire E. Murray (1) https://orcid.org/0000-0002-7743-8129

W. M. Goss (1) https://orcid.org/0000-0001-6596-8803

John M. Dickey (i) https://orcid.org/0000-0002-6300-7459

Brian Babler (10 https://orcid.org/0000-0002-6984-5752

Chang-Goo Kim (10) https://orcid.org/0000-0003-2896-3725

\section{References}

Astropy Collaboration, Robitaille, T. P., Tollerud, E. J., et al. 2013, A\&A, 558, A33

Audit, E., \& Hennebelle, P. 2005, A\&A, 433, 1

Carilli, C. L., Dwarakanath, K. S., \& Goss, W. M. 1998, ApJL, 502, L79

Chengalur, J. N., Kanekar, N., \& Roy, N. 2013, MNRAS, 432, 3074

Clark, B. G. 1965, ApJ, 142, 1398

Condon, J. J., Cotton, W. D., Greisen, E. W., et al. 1998, AJ, 115, 1693

Crovisier, J., Kazes, I., \& Aubry, D. 1978, A\&AS, 32, 205

Dalgarno, A., \& McCray, R. A. 1972, ARA\&A, 10, 375

Dickey, J. M., \& Benson, J. M. 1982, AJ, 87, 278

Dickey, J. M., McClure-Griffiths, N., Gibson, S. J., et al. 2013, PASA, 30, e003

Dickey, J. M., Mebold, U., Stanimirovic, S., \& Staveley-Smith, L. 2000, ApJ, 536, 756

Dickey, J. M., Salpeter, E. E., \& Terzian, Y. 1977, ApJL, 211, L77

Dickey, J. M., Terzian, Y., \& Salpeter, E. E. 1978, ApJS, 36, 77

Draine, B. T. 2011, Physics of the Interstellar and Intergalactic Medium (Princeton, NJ: Princeton Univ. Press)

Dwarakanath, K. S., Carilli, C. L., \& Goss, W. M. 2002, ApJ, 567, 940

Ewen, H. I., \& Purcell, E. M. 1951, Natur, 168, 356

Ferrière, K. M., Zweibel, E. G., \& Shull, J. M. 1988, ApJ, 332, 984

Field, G. B. 1958, PIRE, 46, 240

Fukui, Y., Torii, K., Onishi, T., et al. 2015, ApJ, 798, 6

Greisen, E. W. 2003, ASSL, 285, 109

Hagen, J. P., Lilley, A. E., \& McClain, E. F. 1955, ApJ, 122, 361

Hartmann, D., \& Burton, W. B. 1997, Atlas of Galactic Neutral Hydrogen (Cambridge: Cambridge Univ. Press)

Haud, U., \& Kalberla, P. M. W. 2007, A\&A, 466, 555

Heiles, C., \& Troland, T. H. 2003a, ApJS, 145, 329

Heiles, C., \& Troland, T. H. 2003b, ApJ, 586, 1067

Hughes, M. P., Thompson, A. R., \& Colvin, R. S. 1971, ApJS, 23, 323

Hunter, J. D. 2007, CSE, 9, 90

Kalberla, P. M. W., Burton, W. B., Hartmann, D., et al. 2005, A\&A, 440, 775

Kalberla, P. M. W., \& Haud, U. 2015, A\&A, 578, A78

Kalberla, P. M. W., \& Haud, U. 2018, arXiv: 1806.04085

Kalberla, P. M. W., McClure-Griffiths, N. M., Pisano, D. J., et al. 2010, A\&A, 521, A17

Kim, C.-G., Ostriker, E. C., \& Kim, W.-T. 2013, ApJ, 776, 1
Kim, C.-G., Ostriker, E. C., \& Kim, W.-T. 2014, ApJ, 786, 64

Lazareff, B. 1975, A\&A, 42, 25

Lee, M.-Y., Stanimirović, S., Murray, C. E., Heiles, C., \& Miller, J. 2015, ApJ, 809,56

Lindner, R. R., Vera-Ciro, C., Murray, C. E., et al. 2015, AJ, 149, 138

Liszt, H. 2001, A\&A, 371, 698

Mac Low, M.-M., Balsara, D. S., Kim, J., \& de Avillez, M. A. 2005, ApJ, 626,864

McClure-Griffiths, N. M., Pisano, D. J., Calabretta, M. R., et al. 2009, ApJS, 181,398

McClure-Griffiths, N. M., Stanimirovic, S., Murray, C., et al. 2015, in Advancing Astrophysics with the Square Kilometre Array (AASKA14), The Hydrogen Universe (Trieste: SISSA), 130

McKee, C. F., \& Ostriker, J. P. 1977, ApJ, 218, 148

Mebold, U., Düsterberg, C., Dickey, J. M., Staveley-Smith, L., \& Kalberla, P. 1997, ApJL, 490, L65

Mohan, R., Dwarakanath, K. S., \& Srinivasan, G. 2004, JApA, 25, 185

Muller, C. A., \& Oort, J. H. 1951, Natur, 168, 357

Murray, C. E., Lindner, R. R., Stanimirović, S., et al. 2014, ApJL, 781, L41

Murray, C. E., Peek, J. E. G., Lee, M.-Y., \& Stanimirovic, S. 2018, arXiv: 1806.01300

Murray, C. E., Stanimirović, S., Goss, W. M., et al. 2015, ApJ, 804, 89

Murray, C. E., Stanimirović, S., Kim, C.-G., et al. 2017, ApJ, 837, 55

Nguyen, H., Dawson, J. R., Miville-Deschênes, M.-A., et al. 2018, arXiv:1805. 11787

Peek, J. E. G., Babler, B. L., Zheng, Y., et al. 2018, ApJS, 234, 2

Peek, J. E. G., Heiles, C., Douglas, K. A., et al. 2011, ApJS, 194, 20

Planck Collaboration, Abergel, A., Ade, P. A. R., et al. 2014, A\&A, 571, A11

Pritchard, J. R., \& Loeb, A. 2012, RPPh, 75, 086901

Radhakrishnan, V., Murray, J. D., Lockhart, P., \& Whittle, R. P. J. 1972, ApJS, 24, 15

Reach, W. T., Heiles, C., \& Bernard, J.-P. 2017, ApJ, 834, 63

Rohlfs, K., \& Wilson, T. L. 2004, Tools of Radio Astronomy (Berlin: Springer)

Roy, N., Kanekar, N., Braun, R., \& Chengalur, J. N. 2013a, MNRAS, 436, 2325

Roy, N., Kanekar, N., \& Chengalur, J. N. 2013b, MNRAS, 436, 2366

Shaw, G., Ferland, G. J., \& Hubeny, I. 2017, ApJ, 843, 149

Stanimirović, S., \& Heiles, C. 2005, ApJ, 631, 371

Stanimirović, S., Murray, C. E., Lee, M.-Y., Heiles, C., \& Miller, J. 2014, ApJ, 793, 132

Van Der Walt, S., Colbert, S. C., \& Varoquaux, G. 2011, CSE, 13, 22

Vázquez-Semadeni, E., Gazol, A., \& Scalo, J. 2000, ApJ, 540, 271

Verschuur, G. L., \& Magnani, L. 1994, AJ, 107, 287

Vishniac, E. T., \& Lazarian, A. 1999, ApJ, 511, 193

Winkel, B., Kerp, J., Flöer, L., et al. 2016, A\&A, 585, A41

Wolfire, M. G. 2015, HiA, 16, 600

Wolfire, M. G., McKee, C. F., Hollenbach, D., \& Tielens, A. G. G. M. 2003, ApJ, 587, 278

Wouthuysen, S. A. 1952, AJ, 57, 31 